\title{
THE NUCLEAR NEAR-INFRARED SPECTRAL PROPERTIES OF NEARBY GALAXIES
}

\author{
R. E. Mason ${ }^{1}$, A. Rodríguez-Ardila ${ }^{2}$, L. Martins ${ }^{3}$, R. Riffel ${ }^{4}$, O. González Martín ${ }^{5,6}$, C. Ramos Almeida ${ }^{5,6}$, \\ D. Ruschel Dutra ${ }^{4,5}$, L. C. Ho ${ }^{7,8}$, K. Thanjavur ${ }^{9}$, H. Flohic ${ }^{10}$, A. Alonso-Herrero ${ }^{11}$, P. Lira ${ }^{12}$, R. McDermid ${ }^{1,13,14}$, \\ R. A. Riffel ${ }^{15}$, R. P. Schiavon ${ }^{16}$, C. Winge ${ }^{17}$, M. D. Hoenig ${ }^{1}$, and E. Perlman ${ }^{18}$ \\ ${ }^{1}$ Gemini Observatory, Northern Operations Center, 670 North A'ohoku Place, Hilo, HI 96720, USA; rmason@ gemini.edu \\ ${ }^{2}$ Laboratório Nacional de Astrofísica/MCT, Rua dos Estados Unidos 154, Itajubá, MG, Brazil \\ ${ }^{3}$ NAT_-Universidade Cruzeiro do Sul, Rua Galvão Bueno, 868, São Paulo, SP, Brazil \\ ${ }^{4}$ Universidade Federal do Rio Grande do Sul, Instituto de Física, CP 15051, Porto Alegre 91501-970, RS, Brazil \\ 5 Instituto de Astrofísica de Canarias, Calle Vía Láctea, s/n, E-38205, La Laguna, Tenerife, Spain \\ ${ }^{6}$ Departamento de Astrofísica, Universidad de La Laguna, E-38205, La Laguna, Tenerife, Spain \\ ${ }^{7}$ Kavli Institute for Astronomy and Astrophysics, Peking University, Beijing, China \\ ${ }^{8}$ Department of Astronomy, Peking University, Beijing, China \\ ${ }^{9}$ Department of Physics \& Astronomy, University of Victoria, Victoria, BC, V8W 2Y2, Canada \\ ${ }^{10}$ University of the Pacific, Department of Physics, 3601 Pacific Avenue, Stockton, CA 95211, USA \\ ${ }^{11}$ Instituto de Física de Cantabria, CSIC-UC, E-39005 Santander, Spain \\ ${ }^{12}$ Departamento de Astronomía, Universidad de Chile, Casilla 36-D, Santiago, Chile \\ ${ }^{13}$ Department of Physics and Astronomy, Macquarie University, Sydney NSW 2109, Australia \\ ${ }^{14}$ Australian Gemini Office, Australian Astronomical Observatory, P.O. Box 915, Sydney NSW 1670, Australia \\ ${ }^{15}$ Universidade Federal de Santa Maria, Departamento de Física/CCNE, 97105-900, Santa Maria, RS, Brazil \\ ${ }^{16}$ Astrophysics Research Institute, Liverpool John Moores University, IC2, Liverpool Science Park 146 Brownlow Hill, Liverpool L3 5RF, UK \\ ${ }^{17}$ Gemini Observatory, Southern Operations Center, Casilla 603, La Serena, Chile \\ ${ }^{18}$ Department of Physics \& Space Sciences, Florida Institute of Technology, 150 West University Boulevard, Melbourne, FL 32901, USA \\ Received 2014 August 1; accepted 2015 February 10; published 2015 March 24
}

\begin{abstract}
We present spectra of the nuclear regions of 50 nearby $(D=1-92 \mathrm{Mpc}$, median $=20 \mathrm{Mpc})$ galaxies of morphological types E to Sm. The spectra, obtained with the Gemini Near-IR Spectrograph on the Gemini North telescope, cover a wavelength range of approximately $0.85-2.5 \mu \mathrm{m}$ at $R \sim 1300-1800$. There is evidence that most of the galaxies host an active galactic nucleus (AGN), but the range of AGN luminosities $\left(\log \left(L_{2-10} \mathrm{keV}\right.\right.$ [erg $\left.\left.\left.\mathrm{s}^{-1}\right]\right)=37.0-43.2\right)$ in the sample means that the spectra display a wide variety of features. Some nuclei, especially the Seyferts, exhibit a rich emission-line spectrum. Other objects, in particular the type 2 Low Ionization Nuclear Emission Region galaxies, show just a few, weak emission lines, allowing a detailed view of the underlying stellar population. These spectra display numerous absorption features sensitive to the stellar initial mass function, as well as molecular bands arising in cool stars, and many other atomic absorption lines. We compare the spectra of subsets of galaxies known to be characterized by intermediate-age and old stellar populations, and find clear differences in their absorption lines and continuum shapes. We also examine the effect of atmospheric water vapor on the signal-to-noise ratio achieved in regions between the conventional NIR atmospheric windows, which are of potential interest to those planning observations of redshifted emission lines or other features affected by telluric $\mathrm{H}_{2} \mathrm{O}$. Further exploitation of this data set is in progress, and the reduced spectra and data reduction tools are made available to the community.
\end{abstract}

Key words: galaxies: active - galaxies: nuclei - galaxies: Seyfert - galaxies: stellar content - infrared: galaxies

Supporting material: extended figure, figure set

\section{INTRODUCTION}

Near-infrared (NIR; 0.8-2.5 $\mu \mathrm{m}$ ) observations can provide a wealth of information addressing numerous outstanding questions about the physics and lifecycles of galaxies. Many diagnostic lines are present in this wavelength range: $\mathrm{H}_{2}$ and coronal lines can be used to trace molecular gas and superexcited, often outflowing, material; obscured broad-line regions (BLRs) can be revealed; [Fe II] emission may probe shockexcited gas associated with supernova activity or jets; and stellar absorption lines may measure stages of stellar evolution that have less obvious optical signatures, to name just a few examples. NIR observations of inactive galaxies, starbursts, and active galactic nuclei (AGNs) have already given rise to advances in areas from stellar population studies (e.g., Rieke et al. 1980; McLeod et al. 1993; Origlia et al. 1993; Riffel et al. 2009; Zibetti et al. 2013; Martins et al. 2013a) to black hole (BH) mass measurements (Houghton et al. 2006; Landt et al. 2013; Riffel et al. 2013b).

One topic of much recent interest is the contribution of thermally pulsing asymptotic giant branch (TP-AGB) stars to the NIR luminosity of galaxies. The complexity of this stage of stellar evolution, which occurs in stars of $M \lesssim 6 M_{\odot}$ at ages of $\sim 0.3-2 \mathrm{Gyr}$, makes it very difficult to model accurately (e.g., Marigo et al. 2008), and the available observed spectra of single stars (Rayner et al. 2009 and references therein) span only a limited range of age and metallicity. Whereas some models predict only weak spectral features and a minor contribution from TP-AGB stars to the NIR luminosity of a galaxy (Bruzual \& Charlot 2003), others predict that these stars will cause strong molecular absorptions and contribute as much as $80 \%$ of the K-band light (Maraston 2005). This can have a large effect on the stellar masses and stellar population ages derived from observations (Kannappan \& Gawiser 2007; 
Melbourne et al. 2012). Distinguishing between these models is not straightforward: recent studies have come to very different conclusions regarding whether a large contribution from TP-AGB stars is required (Maraston et al. 2006; Miner et al. 2011; Riffel et al. 2011b; Lyubenova et al. 2012) or ruled out (Kriek et al. 2010; Zibetti et al. 2013) by observations of globular clusters, post-starburst galaxies, and high-redshift galaxies. High-quality NIR spectra of galaxies can be used to search for the molecular absorption bands, such as $\mathrm{CN}, \mathrm{C}_{2}$, and $\mathrm{H}_{2} \mathrm{O}$, expected from these stars (e.g., Riffel et al. 2007). Detailed investigations of these bands in nearby galaxies with a range of properties will aid the development of the models necessary to understand and predict the spectral energy distributions (SEDs) of galaxies at $z \sim 3$, at the age when their initial stellar population was producing large numbers of TP-AGB stars.

Toward the opposite end of the stellar luminosity range, it has been reported that the centers of elliptical galaxies contain an enhanced fraction of low-mass stars, indicating that the stellar initial mass function (IMF) may not be universal (e.g., van Dokkum \& Conroy 2010; Cappellari et al. 2012; La Barbera et al. 2013; Spiniello et al. 2014). The relative numbers of high- and low-mass stars produced during an episode of star formation will affect the influence of the star-forming region on its surroundings, as well as determine the chemical evolution of a galaxy, making the stellar IMF an important area of study. Much of the evidence for a non-universal IMF has come from studies of stellar absorption lines around $1 \mu \mathrm{m}$, such as the $0.99 \mu \mathrm{m}$ Wing-Ford FeH band which is stronger in dwarf stars than in giants. IMF-sensitive features can also be found throughout the NIR (Ivanov et al. 2004; Rayner et al. 2009; Conroy \& van Dokkum 2012). Modeling of the NIR features, especially in conjunction with optical spectra to break the degeneracy between the effects of the IMF and metallicity, may be used to infer the presence of a "bottom-heavy" IMF, and perhaps also to constrain the overall shape of the IMF of extragalactic stellar populations (Conroy \& van Dokkum 2012).

Both stellar- and AGN-related activity may play a role in producing the emission lines observed in objects like lowionization nuclear emission region galaxies (LINERs; e.g., Heckman 1980; Ferland \& Netzer 1983; Terlevich \& Melnick 1985; Binette et al. 1994; Dopita \& Sutherland 1996; Ho 2008). Detailed SED studies suggest that the weak AGNs that exist in many LINERs cannot supply sufficient ionizing photons to power the optical emission lines (Eracleous et al. 2010a). Other power sources must therefore contribute, such as jet or supernova shocks, or stellar photoionization. Alonso-Herrero et al. (2000) were able to explain the NIR [Fe II] $1.26 \mu \mathrm{m} / \mathrm{Pa} \beta$ ratio in several LINERs with an aging starburst model, and correlations between line emission and optical stellar surface brightness/luminosity and soft X-ray emission suggest that post-AGB stars are important sources of ionizing photons in many objects (Macchetto et al. 1996; Flohic et al. 2006; Sarzi et al. 2010). In fact, Stasińska et al. (2008) find that post-AGB stars and white dwarfs could account for the placement of LINERs on the "AGN branch" of optical line diagnostic diagrams, and this could also explain their extended line emission (Singh et al. 2013). If the emission lines in LINERs are indeed not directly indicative of the presence of a weak AGN, this would considerably alter our view of AGN demographics. By providing tracers of young stars (e.g., $\mathrm{Pa} \alpha, \mathrm{Pa} \beta$, and $\mathrm{Br} \gamma$ ) and shocked gas (e.g., [Fe II], $\mathrm{H}_{2}$ ), NIR spectra can be used to investigate the significance of these sources of ionization (Larkin et al. 1998; RodríguezArdila et al. 2004, 2005; Riffel et al. 2013a). In higherluminosity active nuclei, high-excitation NIR coronal lines can be used to probe the AGN ionizing continuum and the density of the surrounding medium, as well as outflows and gas-jet interactions (e.g., Oliva et al. 1994; Marconi et al. 1996; Rodríguez-Ardila et al. 2006, 2011; Geballe et al. 2009).

As well as stars and line-emitting gas, hot dust and the accretion disk also leave detectable IR signatures in a significant fraction of AGNs (Ivanov et al. 2000; RodríguezArdila \& Mazzalay 2006; Riffel et al. 2009; Ramos Almeida et al. 2009a; Mor \& Netzer 2012). The IR spectra of many Seyfert 1 nuclei and quasars show an inversion around $1 \mu \mathrm{m}$, interpreted as the minimum between the AGN-heated dust whose emission peaks in the mid-infrared (MIR), and the accretion disk that emits strongly in the optical/UV (e.g., Landt et al. 2011). At low accretion rates, however, this situation is expected to change. The inner region of the accretion disk is predicted to be replaced by an advection-dominated accretion flow (Esin et al. 1997; Narayan \& McClintock 2008), implying that the disk emission should shift redwards, perhaps into the NIR (Ho 2008; Taam et al. 2012). Furthermore, various models suggest that the obscuring torus of the AGN unified model should disappear when the accretion rate becomes sufficiently low (Elitzur \& Shlosman 2006; Vollmer et al. 2008). The dust continuum in such nuclei should therefore be weak. IR studies of the accretion disk and dust have so far been carried out on only a few low-luminosity AGNs, using high-resolution imaging in the NIR and MIR (Asmus et al. 2011; Mason et al. 2012, 2013). Using NIR spectroscopy to reveal the disk and dust emission over a range of AGN luminosities may unearth systematic trends that will allow a better understanding of accretion and obscuration in AGNs at low accretion rates.

Several sizeable compendia of NIR spectra of local galaxies exist in the literature. In addition to a large number of compilations covering individual NIR atmospheric windows (e.g., Kawara et al. 1987; Goldader et al. 1997b; Vanzi \& Rieke 1997; Ivanov et al. 2000; Reunanen et al. 2002; Kotilainen et al. 2012; van der Laan et al. 2013), a handful of groups have presented spectra covering the whole $\sim 0.8-2.5 \mu \mathrm{m}$ range. Mannucci et al. (2001) compiled largeaperture spectra of 28 normal galaxies, creating template spectra of various morphological types and calculating kcorrections for these objects. Riffel et al. (2006) presented an atlas of 47 AGN nuclear spectra in which a rich set of emission lines was revealed, while Landt et al. (2011, 2013) used NIR spectroscopy of 23 broad-line AGNs to estimate the BH masses of those objects. Mould et al. (2012) showed spectra of the central $\sim 1^{\prime \prime}$ of 136 local, radio-detected elliptical galaxies, with a view to future adaptive optics-assisted integral field spectroscopy. Most recently, a NIR atlas of 23 star-forming galaxies was published by Martins et al. (2013a), and their stellar populations analyzed in Martins et al. (2013b).

In this paper, we present Gemini NIR spectroscopy of the nuclei of 50 galaxies, almost all from the Palomar survey (Ho et al. 1995, 1997a). The Palomar galaxy survey provides a spectral atlas of a near-complete, flux-limited $(B<12.5 \mathrm{mag})$ sample of nearby, northern galaxies. The 486 Palomar galaxies were observed in the optical with a $2^{\prime \prime}$ slit at a spectral 
resolution of $R \sim 2000$, and the resulting spectra revealed 420 emission-line objects-Seyferts, LINERs, transition objects (TO; objects with [O I] strength intermediate between $\mathrm{H}_{\text {II }}$ region galaxies and classical LINERs), and $\mathrm{H}$ iI nuclei-tracing nuclear activity at low luminosities. The uniform, statistically significant data set has been an invaluable aid to research on a wide range of issues.

The galaxies selected for this work were chosen from among the Palomar galaxies showing emission lines in their optical spectra, with Seyfert, LINER, or, occasionally, TO classification. The data complement published NIR galaxy spectra in several ways. Some of the more luminous, nearby AGNs in the subsample provide an opportunity to study numerous, strong AGN emission lines. In most of the galaxies, however, the emission lines are weak and the continuum emission is dominated by starlight. This, together with the good signalto-noise ratio $(\mathrm{S} / \mathrm{N})$ of the spectra, allows detailed study of undiluted atomic and molecular stellar absorption features. The wide, simultaneous wavelength coverage, in some cases with good enough telluric line removal to reveal the regions between the conventional $J$-, $H$-, and $K$-band atmospheric windows, offers an extensive overview of the properties of nearby galaxy nuclei in the NIR. And, as the Palomar galaxies are bright and nearby, it has been possible to obtain high-quality NIR spectra with a relatively modest investment of telescope time.

In Section 2, we describe the sample of galaxies selected for this study. Section 3 gives details about the observations and data reduction, as well as information about accessing the spectra and the code used to produce them. In Section 4 we show some illustrative spectra, compare the absorption-line and continuum spectra of intermediate-age and old galaxies, and discuss the emission lines observed in the data. Additional material, including notes about individual objects, and the optical and NIR spectrum of each one, is given in Appendix A. In Appendix B, we examine how the quality of the data between the NIR atmospheric windows depends on the atmospheric water vapor column at the time of the observations. The intent of this paper is to present the spectra, point out some noteworthy features of the data, and act as a useful reference for the general NIR properties of local galaxy nuclei. Further analysis of various aspects of the data set will be presented in forthcoming papers. ${ }^{19}$

\section{THE SAMPLE}

The galaxies selected for this work were drawn from the Palomar nearby galaxy survey (Ho et al. 1995, 1997a). With the exception of NGC 205, an inactive galaxy chosen as an example of an intermediate-age stellar population (Section 4.2), the targets are all classified by Ho et al. (1997a) as Seyferts, LINERs, and TOs. As well as these 48 galaxies, we also observed two non-Palomar objects (1H1934-063 and NGC 7469) in common with Riffel et al. (2006), to compare the results with previously published spectra.

\footnotetext{
19 These include: (1) a comparison of velocity dispersions derived from the calcium triplet and CO band heads (Riffel et al. 2014); (2) modeling of the stellar absorption features in the highest-quality spectra (R. A. Riffel et al., in preparation); (3) analysis of the emission-line spectra (O. González-Martín, in preparation, L. Martins et al., in preparation); (4) presentation of the extended emission-line spectrum of NGC 4388 (A. Rodríguez-Ardila et al., in preparation); (4) the dust and accretion disk emission in the low-luminosity AGN (Biddle, R.E. Mason et al., in preparation); and (5) full stellar population synthesis of the whole data set (R.A. Riffel et al., in preparation).
}

The characteristics of the Palomar emission-line galaxies in this sample, and those of the remainder of the Seyferts, LINERs and TOs in the original Palomar study, are shown in Figure 1. The objects studied here were chosen primarily to extend the range of AGNs with NIR spectra to lower luminosities than currently available. The hard X-ray luminosities of the AGNs are therefore generally low relative to well-known Seyferts such as NGC 1068 and NGC $4151\left(\log \left(L_{2-10 \mathrm{keV}}\left[\mathrm{erg} \mathrm{s}^{-1}\right]\right)=42.8\right.$ and 42.5, respectively; Panessa et al. 2006). They do, however, lie toward the high end of the X-ray luminosity distribution of the Palomar Seyferts/LINERs/TOs as a whole. This is because (i) in future work we are interested in decomposing the spectra into stellar and AGN (accretion disk and dust) components, and recovering AGN emission from seeing-limited spectra of very low-luminosity AGNs may not be possible; and (ii) brighter nuclei were prioritized in order to enable observations in suboptimal conditions (Section 3). The brighter nuclei also tend to be well-studied objects with plentiful ancillary data, potentially allowing fuller exploitation of the data.

The sample contains comparable numbers of Seyfert and LINER nuclei of both types 1 and 2, and their host galaxies span a range of morphological types from $\mathrm{E}$ to $\mathrm{Sm}$. The distances to the galaxies range from 0.7 to $92 \mathrm{Mpc}$, with a median of $20 \mathrm{Mpc}$. This, and other basic information about each object, is given in Table 1.

\section{OBSERVATIONS AND DATA REDUCTION}

The spectra were obtained using the cross-dispersed (XD) mode of the Gemini Near-IR Spectrograph (GNIRS; Elias et al. 2006) on the $8.1 \mathrm{~m}$ Gemini North telescope. With the "short blue" camera, $321 \mathrm{~mm}^{-1}$ grating and 0.3 slit, this mode gives simultaneous spectral coverage from $\sim 0.83-2.5 \mu \mathrm{m}$ at $R \sim 1300 / 1800^{20}$ with a pixel scale of $0.15 \mathrm{pix}^{-1}$. To minimize the effects of differential atmospheric refraction, which can be important over this wide wavelength range especially at low elevation, the slit was usually orientated close to the mean parallactic angle during the observations of both the science target and standard star. Exceptions are noted in Table 2. Because the galaxies are extended objects, and the slit used in this XD mode is only 7".5 in length, the telescope was nodded to blank sky (typically 50" distant) in an ABBA-type pattern. Individual and total exposure times varied depending on the object's brightness and the likely observing conditions (see below), and are given in Table 2 .

The data were acquired in queue mode between 2011 May and 2013 August. Some observations were taken from standard queue programs, while others came from dedicated poor weather programs, designed to be used in cloudy or poor seeing conditions. The requested (and actual) observing conditions for the objects in this study therefore cover a large range. Some data sets from these programs have been omitted from this paper. Most of these are spectra that were obtained under very poor conditions, resulting in data with very low $\mathrm{S} / \mathrm{N}$ and not useful for the planned scientific analysis. Less commonly, telluric $\mathrm{OH}$ lines did not subtract out well, leaving large residuals in the data, particularly in the $H$ band.

\footnotetext{
${ }^{20}$ Work on the GNIRS grating turret in mid-2012 fixed a problem with the mechanism at the expense of degrading the resolution achieved with the $321 \mathrm{~mm}^{-1}$ grating and 0.13 slit. The spectral resolution of data obtained in that specific configuration before 2012 November was $R \sim 1700$, but has been $R \sim 1300$ since then.
} 

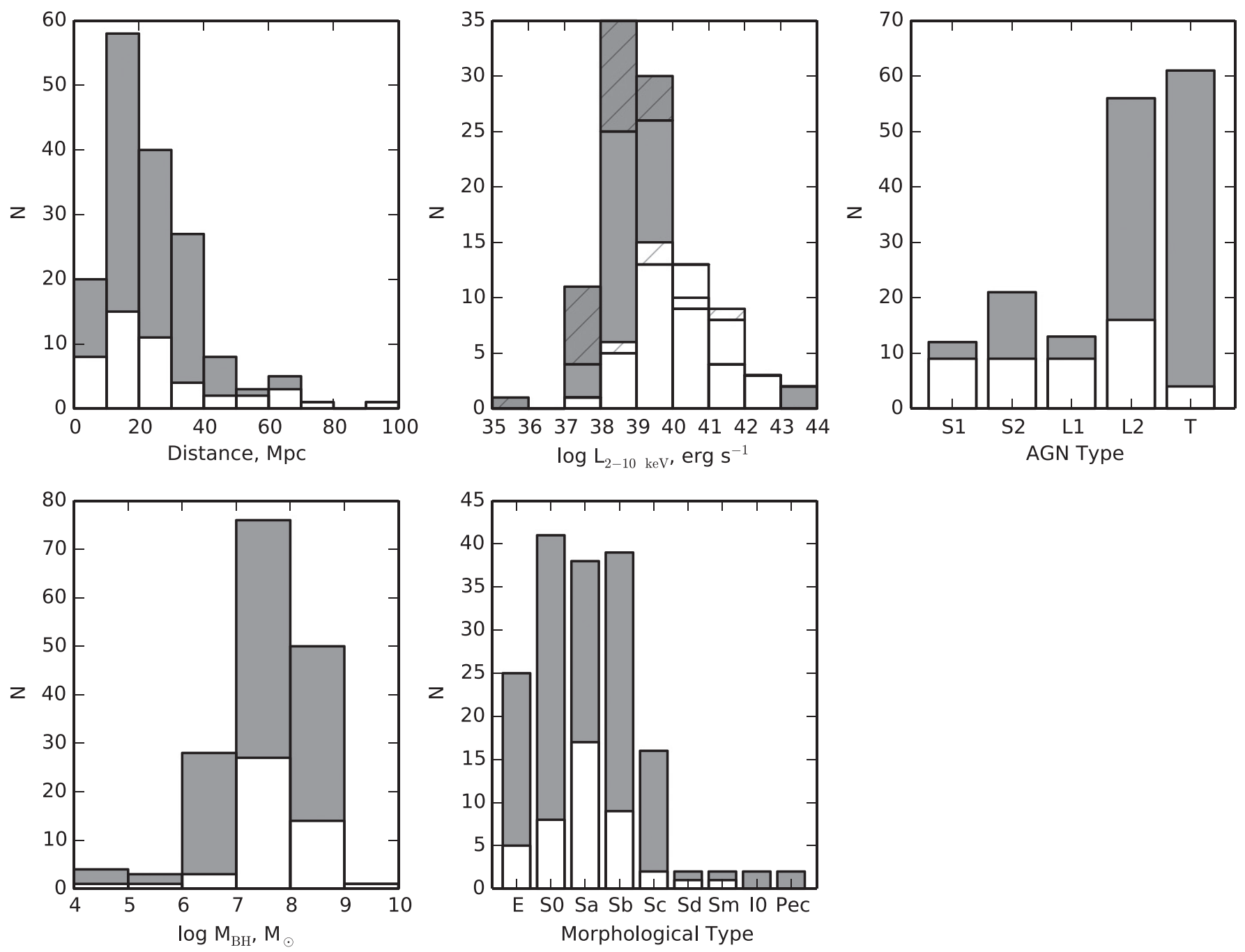

Figure 1. Characteristics of the subsample of Palomar emission-line galaxies presented in this paper (white), and the remainder of the Palomar Seyferts, LINERs, and TOs (gray). Distances, AGN types, and morphological types are from Ho et al. (1997a). "S1" encompasses Seyfert 1-1.9. Black hole masses are derived from the stellar velocity dispersion compilation of Ho et al. (2009), using Equation (3) of Kormendy \& Ho (2013). While estimated hard (2-10 keV) X-ray luminosities are available for all of the Palomar objects in our sample in Ho (2009), the 67 galaxies in the parent sample with no hard X-ray luminosity in that paper are omitted from the $\log L_{X}$ panel. Those objects probably lie toward the low end of the $L_{X}$ distribution. Hatched areas in the $\log L_{X}$ panel indicate upper limits.

The data were reduced using version 1.9 of the "XDGNIRS" pipeline. The code, created for this project and based mainly on the Gemini IRAF package, produces a 1D, roughly fluxcalibrated spectrum from a set of raw science and calibration files. First, the data are cleaned of any electronic striping. "Radiation events," cosmic ray-like features caused by $\alpha$ particles from the coatings of the camera lenses, are identified by comparing each $2 \mathrm{D}$ image to a relatively clean minimum image formed from all data taken at that nod position, and interpolated over. The files are then divided by a flat field constructed from observations of quartz halogen and infrared lamps taken immediately after the galaxy spectroscopy. Following sky subtraction, the 2D data are rectified using daytime pinhole flats. GNIRS' detector is slightly tilted such that sky and arc lines do not fall exactly along detector rows, and this tilt is removed using observations of an argon arc lamp taken along with the science data at night. The arc spectra are also used to wavelength calibrate the data at this stage, and a spectrum of each spectral order is then extracted. For this work, an aperture of $1^{\prime \prime} .8$ along the slit was used, corresponding to about twice the typical seeing in the $K$ band (Table 2). Additional spectra were extracted in steps along the slit, but we do not discuss them in this paper.

To cancel out telluric absorption lines in the data, a standard star, usually of spectral type A0-A5, was observed immediately before or after each galaxy. Prior to using a star to correct the galaxy spectrum, its intrinsic absorption lines are removed either by using a model spectrum of Vega ${ }^{21}$ or by automatically fitting Lorentz profiles to the lines. XDGNIRS allows the user to interactively improve the results from the chosen automatic algorithm, and this option was used in most cases. This process can leave residuals, and we found this particularly common around the wavelengths of $\mathrm{Pa} \beta \quad(1.282 \mu \mathrm{m})$ and $\mathrm{Br} 10$ $(1.737 \mu \mathrm{m})$. The detection and interpretation of weak $\mathrm{H}$ recombination lines in the spectra should be treated with some caution.

IRAF's "telluric" task is then used to shift and scale the standard star spectrum, thereby optimizing the telluric line

\footnotetext{
${ }^{21}$ http://kurucz.harvard.edu/stars.html
} 
Table 1

Basic Information About the Galaxies

\begin{tabular}{|c|c|c|c|c|c|c|c|}
\hline Galaxy & Distance (Mpc) & Morph. Type & Inclination $\left(^{\circ}\right)$ & Activity & $\log L_{2-10 \mathrm{keV}^{\mathrm{a}}}$ & $\log M_{\mathrm{BH}}{ }^{\mathrm{b}}$ & Ref. $^{c}$ \\
\hline \multicolumn{8}{|c|}{ Seyfert $1.2-1.9$} \\
\hline NGC 4235 & 35.1 & $\mathrm{SA}(\mathrm{s}) \mathrm{a}$ & 84 & $\mathrm{~S} 1.2$ & 42.25 & 8.0 & 13,14 \\
\hline NGC 3031 & 1.4 & $\mathrm{SA}(\mathrm{s}) \mathrm{ab}$ & 60 & S 1.5 & 39.38 & 8.1 & $3,6,7$ \\
\hline NGC 5033 & 18.7 & $\mathrm{SA}(\mathrm{s}) \mathrm{c}$ & 64 & S1.5 & 40.70 & 8.0 & 14,21 \\
\hline NGC 4395 & 3.6 & $\mathrm{SA}(\mathrm{s}) \mathrm{m}:$ & 34 & $\mathrm{~S} 1.8$ & 39.58 & 4.9 & $\ldots$ \\
\hline NGC 2639 & 42.6 & $(\mathrm{R}) \mathrm{SA}(\mathrm{r}) \mathrm{a}$ ? & 54 & S1.9 & 40.85 & 8.3 & 3,13 \\
\hline NGC 4258 & 6.8 & $\mathrm{SAB}(\mathrm{s}) \mathrm{bc}$ & 70 & S1.9 & 40.89 & 7.9 & $6,8,12,14,23$ \\
\hline NGC 4388 & 16.8 & $\mathrm{SA}(\mathrm{s}) \mathrm{b}:$ & 83 & S1.9 & 42.14 & 7.0 & $9,14,15,16,17,18,24$ \\
\hline NGC 4565 & 9.7 & $\mathrm{SA}(\mathrm{s}) \mathrm{b} ?$ & $\cdots$ & S1.9 & 39.56 & 7.8 & 32 \\
\hline NGC 4579 & 16.8 & $\mathrm{SAB}(\mathrm{rs}) \mathrm{b}$ & 38 & S1.9/L1.9 & 41.15 & 8.1 & $3,19,23$ \\
\hline \multicolumn{8}{|c|}{ Seyfert 2} \\
\hline NGC 1167 & 65.3 & SA0- & 32 & S2 & 40.32 & 8.7 & 1,23 \\
\hline NGC 1358 & 53.6 & $\mathrm{SAB}(\mathrm{r}) 0 / \mathrm{a}$ & 38 & $\mathrm{~S} 2$ & 42.68 & 8.7 & 23 \\
\hline NGC 2273 & 28.4 & $\mathrm{SB}(\mathrm{r}) \mathrm{a}:$ & 41 & $\mathrm{~S} 2$ & 40.02 & 7.9 & $1,2,14,24$ \\
\hline NGC 2655 & 24.4 & $\mathrm{SAB}(\mathrm{s}) 0 / \mathrm{a}$ & 34 & S2 & 41.87 & 8.1 & $\cdots$ \\
\hline NGC 3079 & 20.4 & $\mathrm{SB}(\mathrm{s}) \mathrm{c}$ & $\ldots$ & S2 & 38.60 & 8.3 & 8,33 \\
\hline NGC 3147 & 40.9 & $\mathrm{SA}(\mathrm{rs}) \mathrm{bc}$ & 27 & $\mathrm{~S} 2$ & 41.87 & 8.7 & 10 \\
\hline NGC 4725 & 12.4 & $\mathrm{SAB}(\mathrm{r}) \mathrm{ab}$ pec & 46 & S2: & 39.11 & 7.8 & 21 \\
\hline NGC 5194 & 7.7 & $\mathrm{SA}(\mathrm{s}) \mathrm{bc}$ pec & 53 & $\mathrm{~S} 2$ & 41.03 & 7.1 & 12,23 \\
\hline NGC 7743 & 24.4 & $(\mathrm{R}) \mathrm{SB}(\mathrm{s}) 0+$ & 32 & S2 & 39.71 & 7.0 & $1,3,12,23$ \\
\hline \multicolumn{8}{|c|}{ LINER 1.9} \\
\hline NGC 266 & 62.4 & $\mathrm{SB}(\mathrm{rs}) \mathrm{ab}$ & 12 & L1.9 & 40.88 & 8.8 & $\ldots$ \\
\hline NGC 315 & 65.8 & $E+:$ & 52 & L1.9 & 41.63 & 9.3 & 1 \\
\hline NGC 1052 & 17.8 & E4 & $\ldots$ & L1.9 & 41.53 & 8.6 & $1,2,5$ \\
\hline NGC 3718 & 17.0 & $\mathrm{SB}(\mathrm{s})$ a pec & 62 & L1.9 & 40.44 & 8.1 & $\ldots$ \\
\hline NGC 3998 & 21.6 & $\mathrm{SA}(\mathrm{r}) 0 ?$ & 34 & L1.9 & 41.34 & 9.3 & $2,3,11,12$ \\
\hline NGC 4203 & 9.7 & SAB0-: & 21 & L1.9 & 39.69 & 8.2 & 1,21 \\
\hline NGC 4450 & 16.8 & $\mathrm{SA}(\mathrm{s}) \mathrm{ab}$ & 43 & L1.9 & 40.02 & 7.8 & $\ldots$ \\
\hline NGC 4750 & 26.1 & $(\mathrm{R}) \mathrm{SA}(\mathrm{rs}) \mathrm{ab}$ & 24 & L1.9 & 39.94 & 7.8 & $\cdots$ \\
\hline NGC 5005 & 21.3 & $\mathrm{SAB}(\mathrm{rs}) \mathrm{bc}$ & 63 & L1.9 & 39.94 & 8.2 & 21 \\
\hline \multicolumn{8}{|c|}{ LINER 2} \\
\hline NGC 404 & 2.4 & $\mathrm{SA}(\mathrm{s}) 0-\mathrm{:}$ & $\ldots$ & L2 & 37.02 & 5.4 & $2,4,12$ \\
\hline NGC 474 & 32.5 & $\left(\mathrm{R}^{\prime}\right) \mathrm{SA}(\mathrm{s}) 0$ & 27 & $\mathrm{~L} 2::$ & $<38.36$ & 8.1 & 1 \\
\hline NGC 1961 & 53.1 & $\mathrm{SAB}(\mathrm{rs}) \mathrm{c}$ & 51 & $\mathrm{~L} 2$ & 40.31 & 8.9 & $\cdots$ \\
\hline NGC 2768 & 23.7 & E6: & $\ldots$ & $\mathrm{L} 2$ & 40.59 & 8.3 & 1 \\
\hline NGC 2832 & 91.6 & $E+2:$ & $\ldots$ & $\mathrm{L} 2::$ & $<41.49$ & 9.5 & $\ldots$ \\
\hline NGC 3169 & 19.7 & $\mathrm{SA}(\mathrm{s}) \mathrm{a}$ pec & 52 & $\mathrm{~L} 2$ & 41.05 & 8.4 & $\cdots$ \\
\hline NGC 3190 & 22.4 & $\mathrm{SA}(\mathrm{s}) \mathrm{a}$ pec & 72 & L2 & 39.54 & 8.4 & $\ldots$ \\
\hline NGC 3607 & 19.9 & $\mathrm{SA}(\mathrm{s}) 0$ & 62 & $\mathrm{~L} 2$ & 38.63 & 8.8 & 1,6 \\
\hline NGC 4346 & 17.0 & SA0 & 70 & L2:: & $<39.82$ & 7.9 & $\ldots$ \\
\hline NGC 4548 & 16.8 & $\mathrm{SB}(\mathrm{rs}) \mathrm{b}$ & 38 & $\mathrm{~L} 2$ & 39.74 & 7.4 & $\cdots$ \\
\hline NGC 4594 & 20.0 & $\mathrm{SA}(\mathrm{s}) \mathrm{a}$ & 68 & L2 & 40.69 & 8.9 & 20 \\
\hline NGC 4736 & 4.3 & $(\mathrm{R}) \mathrm{SA}(\mathrm{r}) \mathrm{ab}$ & 36 & L2 & 38.48 & 7.4 & 7,12 \\
\hline NGC 5371 & 37.8 & $\mathrm{SAB}(\mathrm{rs}) \mathrm{bc}$ & 38 & $\mathrm{~L} 2$ & 40.84 & 8.3 & 21 \\
\hline NGC 5850 & 28.5 & $\mathrm{SB}(\mathrm{r}) \mathrm{b}$ & 30 & L2 & $<39.54$ & 7.8 & 21 \\
\hline NGC 6500 & 39.6 & SAab: & 43 & L2 & 39.73 & 8.6 & 2 \\
\hline NGC 7217 & 16.0 & $(\mathrm{R}) \mathrm{SA}(\mathrm{r}) \mathrm{ab}$ & 34 & L2 & 39.87 & 7.8 & 12 \\
\hline \multicolumn{8}{|c|}{ Transition Object, Inactive } \\
\hline NGC 410 & 70.6 & $E+:$ & $\cdots$ & $\mathrm{T} 2:$ & 40.09 & 9.3 & 1,5 \\
\hline NGC 660 & 11.8 & $\mathrm{SB}(\mathrm{s})$ a pec & 70 & $\mathrm{~T} 2 / \mathrm{H}:$ & 39.55 & 7.6 & $\ldots$ \\
\hline NGC 4569 & 16.8 & $\mathrm{SAB}(\mathrm{rs}) \mathrm{ab}$ & 65 & $\mathrm{~T} 2$ & 39.41 & 7.8 & $2,3,19$ \\
\hline NGC 7331 & 14.3 & $\mathrm{SA}(\mathrm{s}) \mathrm{b}$ & 72 & $\mathrm{~T} 2$ & 38.66 & 7.8 & 22 \\
\hline NGC 205 & 0.7 & dE5 pec & $\cdots$ & $\cdots$ & $<36.41$ & 4.4 & $\cdots$ \\
\hline
\end{tabular}


Table 1

(Continued)

\begin{tabular}{lclccccc}
\hline \hline Galaxy & Distance $(\mathrm{Mpc})$ & Morph. Type & Inclination $\left(^{\circ}\right)$ & Activity & $\log L_{2-10 \mathrm{keV}^{\mathrm{a}}}$ & $\log _{\mathrm{BH}}^{\mathrm{b}}$ & Ref. $^{\mathrm{c}}$ \\
\hline 1H1934-063 & 42.7 & $\mathrm{E}$ & 35 & $\mathrm{NLS} 1$ & 42.8 & 8.3 & $25,26,28$ \\
NGC 7469 & 57.0 & SBa & 30 & S1 & 43.2 & 7.7 & $8,27,28,29,30,31$ \\
\hline
\end{tabular}

Notes. All data from Ho et al. (1997a) and references therein, except where stated. S = Seyfert, L—LINER, T—Transition Object, H—H II region galaxy. “.” indicates an uncertain classification, "::" a very uncertain one, as in Ho et al. (1997a).

${ }^{a}$ From Ho (2009), who used spectral fitting where available to convert published luminosities to the $2-10 \mathrm{keV}$ bandpass, and otherwise assumed a standard spectral slope and no extinction correction.

${ }^{\mathrm{b}}$ Calculated using the stellar velocity dispersions $\left(\sigma_{*}\right)$ of Ho et al. (2009), and Equation (3) of Kormendy \& Ho (2013).

${ }^{\mathrm{c}}$ Previous spectroscopy in the $1-2.5 \mu \mathrm{m}$ range (including only papers in which the spectra are presented). (1) Mould et al. (2012). (2) Rhee \& Larkin (2005). (3) Alonso-Herrero et al. (2000). (4) Seth et al. (2010). (5) Mobasher \& James (1996). (6) Kang et al. (2013). (7) Walker et al. (1988). (8) Sosa-Brito et al. (2001). (9) Veilleux et al. (1997). (10) Tran et al. (2011). (11) Walsh et al. (2012). (12) Larkin et al. (1998). (13) Imanishi \& Wada (2004). (14) Ivanov et al. (2000). (15) Imanishi \& Alonso-Herrero (2004). (16) Knop et al. (2001). (17) Goodrich et al. (1994). (18) Blanco et al. (1990). (19) Mazzalay et al. (2013).(20) Cesetti et al. (2009). (21) Bendo \& Joseph (2004). (22) Matsuoka et al. (2012). (23) Oi et al. (2010). (24) van der Laan et al. (2013). (25) Rodríguez-Ardila et al. (2000). (26) Rodríguez-Ardila et al. (2002). (27) Riffel et al. (2006). (28) Landt et al. (2008). (29) Davies et al. (2005). (30) Hawarden et al. (1999). (31) Goldader et al. (1997a). (32) Doyon et al. (1989). (33) Hawarden et al. (1995).

d Distances from NED, morphologies and inclinations from Hyperleda (Paturel et al. 2003). NGC 7469: AGN type from Osterbrock \& Martel (1993), X-ray luminosity from Pereira-Santaella et al. (2011). 1H1934-063: AGN type from Rodríguez-Ardila et al. (2000), X-ray luminosity from Panessa et al. (2011). Black hole masses calculated as above, using $\sigma_{*}$ from Nelson et al. (2004) and Riffel et al. (2013b).

removal. NIR telluric absorption is caused by various species; for example, the strong absorption around $1.9 \mu \mathrm{m}$ is largely due to $\mathrm{H}_{2} \mathrm{O}$, whereas the bands between $\sim 2.0-2.1 \mu \mathrm{m}$ are produced by $\mathrm{CO}_{2}$. The best cancellation of one set of bands does not necessarily result in good cancellation of others, and a subjective judgment was made as to the most useful result in these cases.

For the purposes of flux calibration the standard stars are assumed to have the continuum spectrum of a blackbody of the appropriate temperature (Pecaut \& Mamajek 2013). An absolute flux scale is applied by simply scaling the blackbody spectrum to the 2MASS $K s$-band magnitude of the standard star (Skrutskie et al. 2006). To estimate the accuracy of the flux calibration we identified several nights on which more than one standard star was observed for our program, and used one star on each night to flux calibrate the other(s). On clear nights the resulting magnitudes differed by $5-40 \%$ from 2MASS, with errors likely dominated by seeing variations in the 0. ". 3 slit.

The individual spectral orders generally match well in flux, with the exception of fairly frequent small (few per cent) offsets between orders 7 and 8. XDGNIRS allows interactive scaling of the orders to remove any inter-order offsets, and also editing of the range of pixels from each order that is used to create the final, combined spectrum. These operations were carried out in a few cases. On rare occasions an obvious, spurious artefact remained in the final spectrum, usually resulting from imperfect removal of a radiation event. These were interpolated over at the end of the reduction.

The raw and reduced data are available through the Canadian Advanced Network for Astronomical Research (CANFAR), at http://www.canfar.phys.uvic.ca/vosui/ \#/karun/xdgnirs_Dec2014. An account with the Canadian Astronomy Data Center is needed to access the storage area, and can be obtained from http://3.cadc-ccda.hia-iha.nrc-cnrc. gc.ca/en/auth/request.html. The data files are accompanied by "readme" files that contain the commands used to reduce the data. For readers who may be interested in, for example, reextracting spectra in different apertures, the latest version of the XDGNIRS code and manual can be found on the Gemini data reduction forum: http://drforum.gemini.edu/forums/ gemini-data-reduction.

\section{RESULTS}

In this section we show some illustrative spectra and discuss the overall features of the data set. The individual galaxies and their spectra are discussed in more depth in Appendix A.

\subsection{General Characteristics}

Three example spectra are presented in Figure 2. NGC 2768 is typical of the LINER 2 and TO classes: with few exceptions, these objects exhibit a blue continuum, many stellar absorption lines, and few or no detectable emission lines. The line emission in the LINER 1.9 and Seyfert 2 classes is more prominent, but on the whole they also tend to contain few, weak emission lines. With strong lines from both low- and high-excitation species, NGC 2273 (S2) is a notable exception. The Seyfert 1.2-1.9 class is the most varied in this sample. It contains objects with few, weak emission lines (NGC 4258, NGC 5033); with numerous strong, relatively narrow lines (NGC 4395, NGC 4388); and one object (NGC 4235) with very broad, asymmetric features.

With some exceptions, the continuum emission of the galaxies generally slopes down toward longer wavelengths (in $\mathrm{F}_{\lambda}$ ). This overall spectral shape is characteristic of stellar emission from the host galaxy of the weak AGN. The red tail of the optical/UV accretion disk emission, and hot dust emission in the $K$ band, often visible in higher-luminosity AGNs (e.g., Riffel et al. 2009; Landt et al. 2011) are not directly detected in most of these objects. This is likely an effect of both AGN luminosity and angular resolution; for instance, previous work has shown that hot dust is detected in adaptive optics-assisted spectroscopy of Mrk 1066, but not in seeing-limited data (Riffel et al. 2010). A minority of the spectra exhibit a flattening or turnover at short wavelengths. This is most pronounced in NGC 1961, NGC 3079, NGC 3718, and NGC 4388. These are all spiral galaxies with dust lanes crossing the line of sight (see notes in Appendix A), suggesting that this spectral shape is due to extinction.

A broad, weak "bump" is frequently visible in the $H$ band superimposed on the general stellar continuum and extending from about $1.45-1.75 \mu \mathrm{m}$. This likely results from a minimum in the opacity of $\mathrm{H}^{-}$in cool stars in the galaxies (Rayner 
Table 2

Observing Log

\begin{tabular}{|c|c|c|c|c|c|c|c|}
\hline Galaxy & Program ID & Date $^{a}$ & $T \exp (s) \times N \exp$ & $\begin{array}{c}\text { Seeing } \\
\prime \prime\end{array}$ & Clear? $^{\mathrm{c}}$ & $\begin{array}{l}\text { Slit angle } \\
{ }^{\circ} \mathrm{E} \text { of } \mathrm{N}\end{array}$ & $\begin{array}{c}\text { Aperture }^{\mathrm{d}} \\
\mathrm{pc}\end{array}$ \\
\hline \multicolumn{8}{|c|}{ Seyfert $1.2-1.9$} \\
\hline NGC 4235 & GN-2013A-Q-16 & 2013 Feb 02 & $240 \times 4$ & 0.76 & $\mathrm{Y}$ & 293 & $51 \times 306$ \\
\hline NGC 3031 & GN-2012A-Q-23 & 2012 Mar 05 & $120 \times 6$ & 0.92 & $\mathrm{Y}$ & 194 & $2 \times 12$ \\
\hline NGC 5033 & GN-2012A-Q-23 & 2012 Mar 01 & $120 \times 6$ & 0.62 & $\mathrm{~N}$ & 130 & $27 \times 163$ \\
\hline NGC 4395 & GN-2012B-Q-80 & 2012 Dec 16 & $240 \times 4$ & 0.84 & $\mathrm{Y}$ & 267 & $5 \times 31$ \\
\hline NGC 2639 & GN-2012A-Q-23 & 2012 Jan 30 & $120 \times 6$ & 1.00 & $\mathrm{Y}$ & $133^{\mathrm{e}}$ & $62 \times 372$ \\
\hline NGC 4258 & GN-2012A-Q-23 & 2012 May 31 & $120 \times 6$ & 0.77 & $\mathrm{~N}$ & 130 & $10 \times 59$ \\
\hline NGC 4388 & GN-2013A-Q-16 & 2013 Feb 04 & $240 \times 4$ & 0.58 & $\mathrm{Y}$ & 64 & $24 \times 147$ \\
\hline NGC 4565 & GN-2013A-Q-16 & 2013 Feb 05 & $240 \times 4$ & 0.71 & $\mathrm{Y}$ & 277 & $14 \times 85$ \\
\hline NGC 4579 & GN-2012A-Q-23 & 2012 Jun 04 & $120 \times 3$ & 0.85 & $\mathrm{~N}$ & $140^{\mathrm{e}}$ & $24 \times 147$ \\
\hline \multicolumn{8}{|c|}{ Seyfert 2} \\
\hline NGC 1167 & GN-2011B-Q-111 & 2011 Dec 04 & $120 \times 6$ & 0.46 & $\mathrm{~N}$ & 97 & $95 \times 570$ \\
\hline NGC 1358 & GN-2011B-Q-111 & 2011 Dec 05 & $120 \times 6$ & 0.69 & $\mathrm{~N}$ & 90 & $78 \times 468$ \\
\hline NGC 2273 & GN-2012B-Q-112 & 2012 Dec 04 & $240 \times 4$ & 0.66 & $\mathrm{Y}$ & 169 & $41 \times 248$ \\
\hline NGC 2655 & GN-2013A-Q-16 & 2013 Feb 12 & $240 \times 4$ & 0.86 & $\mathrm{Y}$ & 177 & $36 \times 213$ \\
\hline NGC 3079 & GN-2012A-Q-23 & 2012 Jan 23 & $120 \times 6$ & 0.67 & $\mathrm{Y}$ & 180 & $30 \times 178$ \\
\hline NGC 3147 & GN-2012B-Q-80 & 2012 Dec 15 & $240 \times 4$ & 1.01 & $\mathrm{Y}$ & 207 & $60 \times 357$ \\
\hline NGC 4725 & GN-2012A-Q-120 & 2012 May 30 & $300 \times 6$ & 1.15 & $\mathrm{~N}$ & 100 & $18 \times 108$ \\
\hline NGC 5194 & GN-2012A-Q-23 & 2012 May 31 & $120 \times 6$ & 0.55 & $\mathrm{~N}$ & 190 & $11 \times 67$ \\
\hline NGC 7743 & GN-2012A-Q-120 & 2012 Jun 07 & $300 \times 4$ & 0.82 & $\mathrm{~N}$ & 290 & $36 \times 213$ \\
\hline \multicolumn{8}{|c|}{ LINER 1.9} \\
\hline NGC 266 & GN-2012B-Q-112 & 2012 Nov 13 & $240 \times 4$ & 0.65 & $\mathrm{Y}$ & 210 & $91 \times 545$ \\
\hline NGC 315 & GN-2012B-Q-112 & 2012 Nov 15 & $240 \times 4$ & 0.69 & $\mathrm{~N}$ & 0 & $96 \times 574$ \\
\hline NGC 1052 & GN-2011B-Q-111 & 2011 Dec 07 & $120 \times 6$ & 0.61 & $\mathrm{~N}$ & $90^{\mathrm{e}}$ & $26 \times 155$ \\
\hline NGC 3718 & GN-2013A-Q-16 & 2013 Feb 02 & $240 \times 4$ & 0.83 & $\mathrm{Y}$ & 241 & $25 \times 148$ \\
\hline NGC 3998 & GN-2012A-Q-23 & 2013 Jan 01 & $120 \times 6$ & 0.78 & $\mathrm{~N}$ & 0 & $31 \times 189$ \\
\hline NGC 4203 & GN-2012A-Q-23 & 2013 Jan 02 & $120 \times 6$ & 0.51 & $\mathrm{~N}$ & 17 & $14 \times 85$ \\
\hline NGC 4450 & GN-2012A-Q-23 & 2012 May 01 & $120 \times 6$ & 0.96 & $\mathrm{Y}$ & 79 & $24 \times 147$ \\
\hline NGC 4750 & GN-2013A-Q-16 & 2013 Feb 15 & $240 \times 4$ & 0.81 & $\mathrm{Y}$ & $227^{\mathrm{e}}$ & $38 \times 228$ \\
\hline NGC 5005 & GN-2013A-Q-16 & 2013 Feb 15 & $240 \times 4$ & 0.75 & $\mathrm{Y}$ & 260 & $31 \times 186$ \\
\hline \multicolumn{8}{|c|}{ LINER 2} \\
\hline NGC 404 & GN-2011B-Q-111 & 2011 Oct 31 & $120 \times 6$ & 0.55 & $\mathrm{~N}$ & 125 & $4 \times 21$ \\
\hline NGC 474 & GN-2012B-Q-112 & 2012 Nov 09 & $300 \times 2$ & 0.88 & $\mathrm{~N}$ & 60 & $47 \times 284$ \\
\hline NGC 1961 & GN-2012B-Q-112 & 2012 Dec 04 & $240 \times 4$ & 0.66 & $\mathrm{Y}$ & 166 & $77 \times 463$ \\
\hline NGC 2768 & GN-2012B-Q-80 & 2012 Nov 15 & $240 \times 4$ & 0.77 & $\mathrm{Y}$ & 240 & $35 \times 207$ \\
\hline NGC 2832 & GN-2013A-Q-16 & 2013 Feb 12 & $240 \times 4$ & 0.90 & $\mathrm{Y}$ & 56 & $133 \times 799$ \\
\hline NGC 3169 & GN-2012A-Q-120 & 2012 Apr 18 & $300 \times 6$ & 1.22 & $\mathrm{~N}$ & 63 & $29 \times 172$ \\
\hline NGC 3190 & GN-2011B-Q-111 & 2011 Nov 27 & $120 \times 6$ & 0.89 & $\mathrm{~N}$ & 275 & $37 \times 196$ \\
\hline NGC 3607 & GN-2012A-Q-120 & 2012 Apr 17 & $300 \times 6$ & 1.51 & $\mathrm{Y}$ & 81 & $29 \times 174$ \\
\hline NGC 4346 & GN-2013A-Q-16 & 2013 Feb 02 & $240 \times 4$ & 0.66 & $\mathrm{Y}$ & 226 & $25 \times 148$ \\
\hline NGC 4548 & GN-2013A-Q-16 & 2013 Feb 04 & $240 \times 4$ & 0.59 & $\mathrm{Y}$ & 74 & $24 \times 147$ \\
\hline NGC 4594 & GN-2013A-Q-16 & $2013 \mathrm{Feb} 02$ & $240 \times 4$ & 0.62 & $\mathrm{Y}$ & 31 & $29 \times 175$ \\
\hline NGC 4736 & GN-2011A-Q-126 & 2011 May 16 & $300 \times 2$ & 0.48 & $\mathrm{~N}$ & $357^{\mathrm{e}}$ & $6 \times 38$ \\
\hline NGC 5371 & GN-2013A-Q-16 & 2013 Feb 15 & $240 \times 4$ & 0.68 & $\mathrm{Y}$ & 247 & $55 \times 330$ \\
\hline NGC 5850 & GN-2013A-Q-16 & 2013 Feb 09 & $240 \times 4$ & 0.93 & $\mathrm{Y}$ & 150 & $42 \times 249$ \\
\hline NGC 6500 & GN-2012A-Q-120 & 2012 May 30 & $300 \times 6$ & 0.94 & $\mathrm{~N}$ & 280 & $58 \times 346$ \\
\hline NGC $7217^{\mathrm{f}}$ & GN-2013A-Q-16 & 2013 May $05 / 22$ & $240 \times 4$ & $0.96,0.70$ & $\mathrm{~N}, \mathrm{Y}$ & 266,254 & $23 \times 140$ \\
\hline
\end{tabular}

Transition Object, Inactive

\begin{tabular}{|c|c|c|c|c|c|c|c|}
\hline NGC 410 & GN-2011B-Q-111 & 2011 Oct 31 & $120 \times 6$ & 0.40 & $\mathrm{~N}$ & 110 & $103 \times 616$ \\
\hline NGC 660 & GN-2011B-Q-111 & 2011 Nov 01 & $126 \times 6$ & 0.40 & $\mathrm{~N}$ & 75 & $17 \times 103$ \\
\hline NGC 4569 & GN-2012B-Q-80 & 2012 Dec 17 & $240 \times 4$ & 0.65 & $\mathrm{~N}$ & 285 & $24 \times 147$ \\
\hline NGC 7331 & GN-2011B-Q-111 & 2011 Nov 23 & $120 \times 6$ & 0.46 & $\mathrm{~N}$ & 137 & $21 \times 125$ \\
\hline NGC 205 & GN-2012B-Q-80 & 2012 Dec 02 & $240 \times 4$ & 0.66 & $\mathrm{~N}$ & 227 & $1 \times 6$ \\
\hline
\end{tabular}

Non-Palomar Galaxies

$\begin{array}{llllllll}\text { 1H1934-063 } & \text { GN-2013A-Q-120 } & 2013 \text { Jul } 11 & 120 \times 4 & 0.81 & \text { Y } & 310 & 76 \times 454\end{array}$


Table 2

(Continued)

\begin{tabular}{|c|c|c|c|c|c|c|c|}
\hline Galaxy & Program ID & Date $^{a}$ & $\operatorname{Texp}(s) \times N \exp$ & $\begin{array}{c}\text { Seeing }^{\mathrm{b}} \\
\end{array}$ & Clear? $^{c}$ & $\begin{array}{l}\text { Slit angle } \\
{ }^{\circ} \mathrm{E} \text { of } \mathrm{N}\end{array}$ & $\begin{array}{c}\text { Aperture }^{\mathrm{d}} \\
\mathrm{pc}\end{array}$ \\
\hline NGC 7469 & GN-2013A-Q-120 & 2013 Aug 07 & $120 \times 4$ & 0.76 & $\mathrm{~N}$ & 299 & $62 \times 373$ \\
\hline
\end{tabular}

${ }^{\text {a }}$ Data obtained before 2012 November 01 have $R \sim 1700$, otherwise $R \sim 1300$; see Section 3 for details.

${ }^{\mathrm{b}}$ FWHM of standard star in the $K$ band. For NGC 4395 and NGC 7743, FWHM star $>$ FWHM $_{\text {galaxy }}$, so the galaxy FWHM is given instead.

${ }^{c}$ Whether or not sky was clear at the time of observation, as judged by the observing log and the Mauna Kea All Sky Infrared and Visible Sky Monitor (http://cfht. hawaii.edu/ asiva/).

${ }^{\mathrm{d}}$ Slit width $\times$ extraction aperture.

${ }^{\mathrm{e}}$ Galaxy and/or standard star observed with slit $>15^{\circ}$ from parallactic angle and at airmass $>1.2$. See, e.g., http://gemini.edu/sciops/instruments/gnirs/spectroscopy/ observing-strategies\#refraction for a table giving the magnitude of differential atmospheric refraction effects as a function of wavelength and airmass.

${ }^{\mathrm{f}}$ Spectrum is mean of 2 epochs.

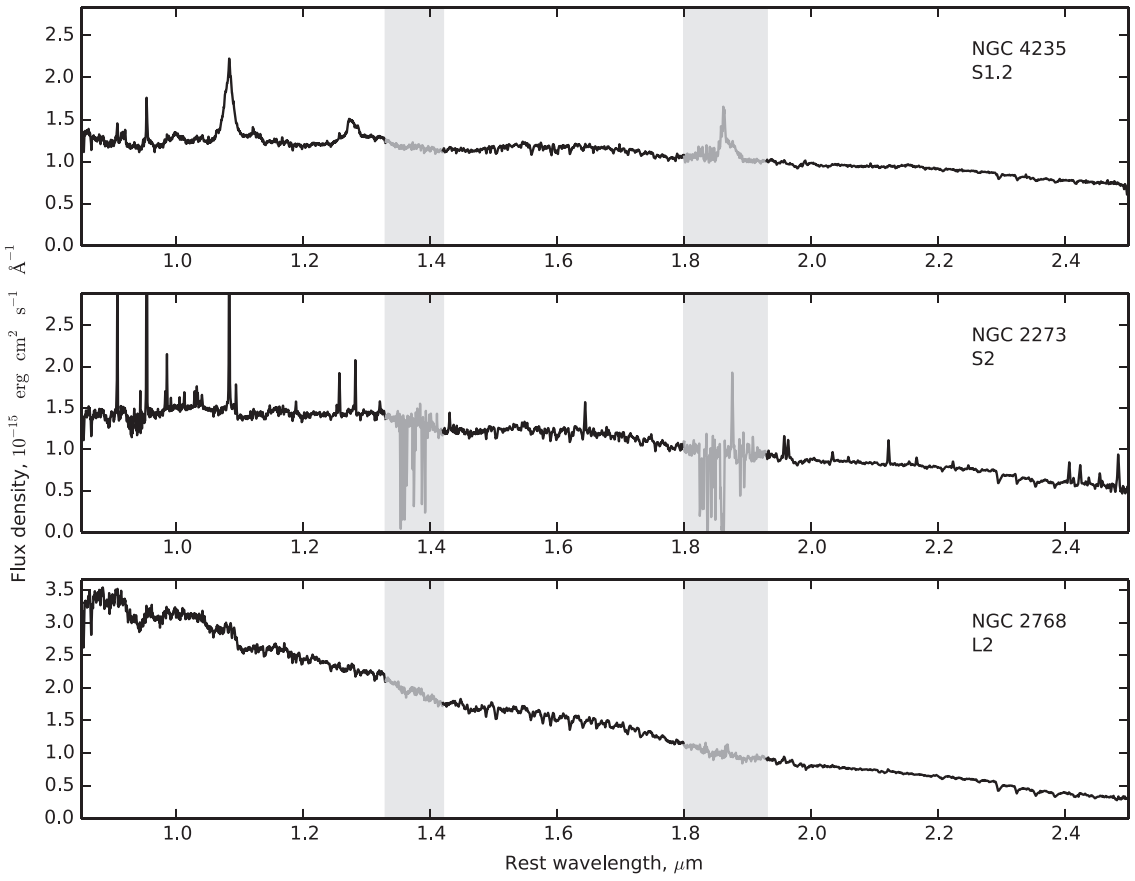

Figure 2. Example spectra. NGC 4235, a type 1.2 Seyfert, shows several broad emission lines while NGC 2273 (type 2 Seyfert) exhibits numerous narrow lines. In contrast, NGC 2768, a LINER 2, has no detectable emission lines and a much bluer continuum. Many absorption features arising in the galaxies' stellar populations are visible. Regions of poor atmospheric transmission $\left(\lesssim 20 \%\right.$ for $3 \mathrm{~mm} \mathrm{H}_{2} \mathrm{O}$ at zenith) are indicated in gray. See Figure 6 and Appendix A for emission line identifications and Figure 3 for absorption lines.

et al. 2009), whose absorption features are discussed below. As can be seen in Figure 2, the quality of the data in the regions around 1.35 and $1.9 \mu \mathrm{m}$, between the $J / H$ and $H / K$ bands, varies widely. This depends primarily on the telluric water vapor column at the time of the observations and is investigated in Appendix B.

\subsection{Absorption Lines}

Numerous stellar absorption lines are present in the spectra. In particular, the optically classified type 2 LINERs often exhibit only few, very weak emission lines in the NIR, clearly revealing the absorption line spectrum of the nuclear stellar population. Figure 3 compares three spiral galaxies (NGC 4565, NGC 5371, and NGC 5850) with high S/N, good telluric line removal, and little detectable line emission, illustrating the absorption features in the spectra. The detailed structure of all three spectra is remarkably similar, revealing weak bands common to all the galaxies. Systematic effects are unlikely to be responsible for the presence of these features at the same rest-frame wavelength in all the spectra. The redshifts of the nearest and most distant of the three objects, $z=0.0041$ (NGC 4565) and $z=0.0085$ (NGC 5371), mean that the shifts necessary to move the spectra to the rest frame differ by about $0.005 \mu \mathrm{m}$. This is approximately the width of the Na I $1.14 \mu \mathrm{m}$ absorption, and should be sufficient to ensure that artifacts from telluric line removal, or pixel-dependent effects (e.g., flatfielding errors), do not affect all three spectra in the same way. In support of this interpretation, Figure 3 also shows a fit of empirical stellar spectra to the spectrum of NGC 5850. The fit was carried out with the STARLIGHT spectral synthesis software (Cid Fernandes et al. 2004, 2005b, 2005a; Asari et al. 2007), using the empirical IRTF NIR stellar spectral library (Rayner et al. 2009) as a base set. ${ }^{22}$ The spectrum is

\footnotetext{
22 The base set also included hotter stars than found in the IRTF library (P. Coelho 2014, private communication), a $F_{\nu} \propto \nu^{-0.5}$ power-law to represent a possible contribution from the AGN featureless continuum, and a set of blackbody spectra to represent hot dust. The aim of the modeling is not to derive information about the stellar population, but simply to investigate which of the spectral features may be due to starlight.
} 


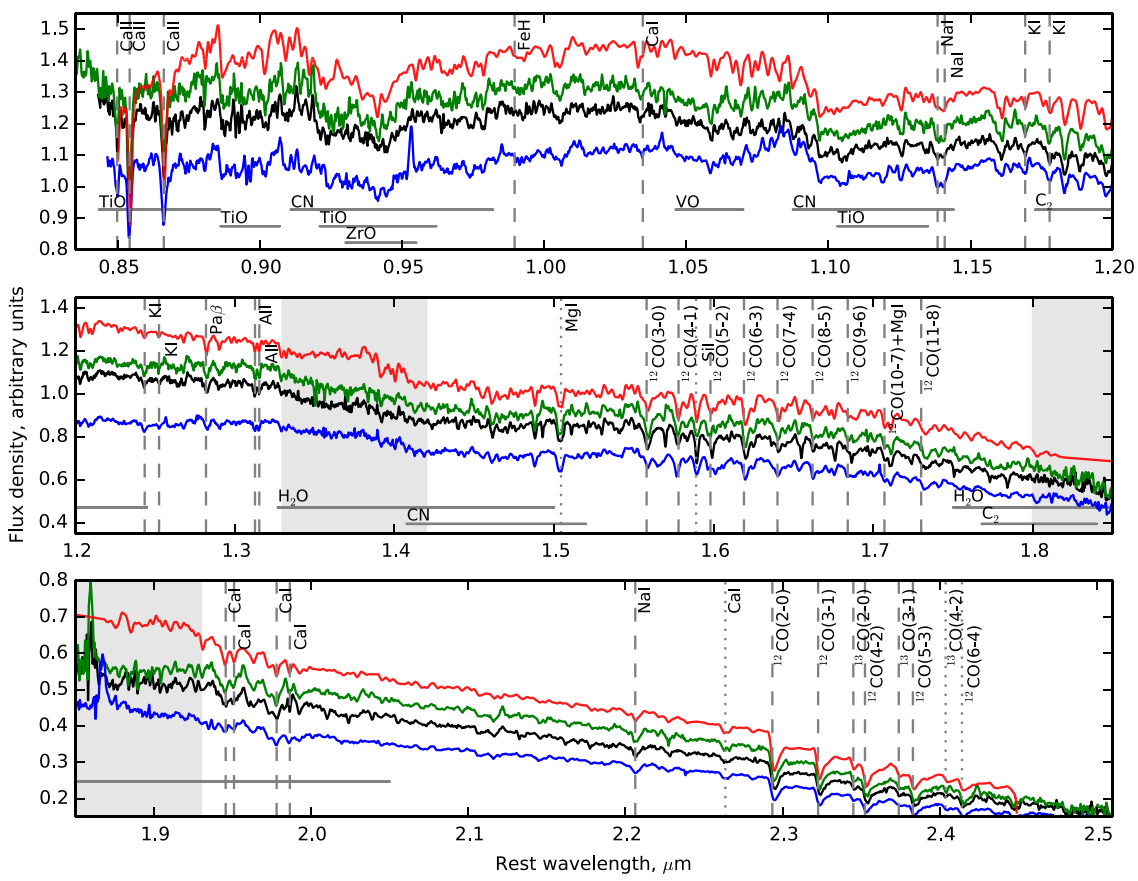

Figure 3. From bottom to top in each panel: Spectra of NGC 4565 (blue), NGC 5371 (black), and NGC 5850 (green), and a fit of empirical stellar spectra to NGC 5850 (red), illustrating various stellar absorption lines. The wavelengths of IMF-sensitive features (see text) are indicated by dashed vertical lines, and those of molecular bands (other than FeH and CO) by solid horizontal lines. Other lines of potential interest are shown by dotted vertical lines. The spectra have been scaled by separate multiplicative factors in each panel for clarity. Regions of poor atmospheric transmission, as defined in Figure 2, are indicated in gray.

very well reproduced by this model fit, implying that the numerous, weak features are real and arise in the stellar populations of the galaxies.

These absorption features have a number of practical applications. For example, in a recent study, Conroy \& van Dokkum (2012) identified several bands in the NIR spectral region that are sensitive to stellar surface gravity and can therefore aid in determining the IMF of a galaxy. The locations of the bands identified in their Figure B1.2 are marked in Figure 3. Most of these lines-from $\mathrm{Ca}$ II, $\mathrm{Ca}$ I, $\mathrm{Na}$ I, $\mathrm{K}$ I, $\mathrm{Pa} \beta$, $\mathrm{Al}$ I, and $\mathrm{CO}$-are not difficult to detect in large-telescope observations of nearby galaxy nuclei; with the exception of the weak, broad $0.99 \mu \mathrm{m} \mathrm{FeH}$ line, they are all clearly visible in the spectra in Figure 3. While features at $\lambda \sim 0.8-1 \mu$ m have been widely used to investigate the IMF in various galaxy samples (e.g., Cenarro et al. 2003; van Dokkum \& Conroy 2010; Smith et al. 2012), to the best of our knowledge the longerwavelength features have not yet been used in this way. Stellar population synthesis modeling of NIR galaxy spectra like these, together with optical spectroscopy to break the degeneracy between the effects of IMF variations and elemental abundances, may be a powerful way of measuring the IMF of local objects. ${ }^{23}$

\footnotetext{
23 The galaxies in this sample host weak active nuclei. In most cases the AGN accretion disk and dust are not bright enough relative to the stellar emission to leave a noticeable imprint on the spectrum (Section 4.1). However, given the small magnitude of the effect of IMF variations on the features, it is possible that weak AGN emission could cause systematic errors in the measurement of their strengths. Fitting the spectra with stellar libraries should reveal whether AGN emission must be present in the nuclear aperture, and extracting extranuclear spectra would entirely avoid the possibility of AGN contamination. We note that the sample of van Dokkum \& Conroy (2010), who analyzed features at $0.8-1.0 \mu \mathrm{m}$, contained some Virgo cluster LINERs. Their results did not depend on aperture size, suggesting that AGN contamination was negligible in their sample.
}

Some other absorption features of potential interest are also indicated in Figure 3. The numerous, relatively strong and evenly spaced features in the $H$ and $K$ bands are $\mathrm{CO}$ rovibrational bands (sometimes blended with bands of other species; Origlia et al. 1993). Velocity dispersions derived from the $\mathrm{CO}$ (and CaT) bands are presented in a companion paper (Riffel et al. 2015). Origlia et al. (1993) characterize the dependence of the equivalent widths of the $\mathrm{CO}(6,3)(1.62 \mu \mathrm{m})$ and $\mathrm{CO}(2,0)(2.29 \mu \mathrm{m})$ bands, as well as the $\mathrm{Si}(1.59 \mu \mathrm{m})$ band, on stellar spectral type, and also demonstrate that the $H$-band $\mathrm{CO}$ lines are much less prone to dilution by AGNheated dust emission than the $K$-band lines. Based on that work, the $\mathrm{Si}(1.59 \mu \mathrm{m}) / \mathrm{CO}(1.62 \mu \mathrm{m})$ equivalent width ratio has been used to derive the average stellar spectral type in Seyfert and inactive spiral galaxies (Ramos Almeida et al. 2009b; Kotilainen et al. 2012). Ivanov et al. (2004) also suggest the use of the $\mathrm{Mg} 1.50 \mu \mathrm{m}$ band as a stellar effective temperature indicator.

As well as the features discussed so far, many broad, shallow molecular absorption features are also apparent in Figure 3. The bands arise in the atmospheres of cool stars- $\mathrm{M}$ dwarfs, giants, and the TP-AGB stars that are of concern to stellar population synthesis models (Section 1). TP-AGB stars are variable, masslosing, stars (oxygen-rich, late-M giants; s-process enhanced Sstars; and the " $\mathrm{N}$ " variety of carbon star; Habing \& Olofsson 2003) in which a thermally unstable He-burning shell causes repeated thermal pulses. The locations of molecular bands (other than $\mathrm{CO}$ ) that are prominent in spectra of late-M giants, $\mathrm{S}$ stars, and C-N stars, as shown by Rayner et al. (2009), are indicated by horizontal lines ${ }^{24}$ in Figure 3.

\footnotetext{
${ }^{24}$ The short-wavelength ends of the bars are located at the band heads quoted by Rayner et al. (2009). Their lengths illustrate the approximate extent of the absorption features, subjectively judged from Figures 34 and 39 of Rayner et al. (2009).
} 


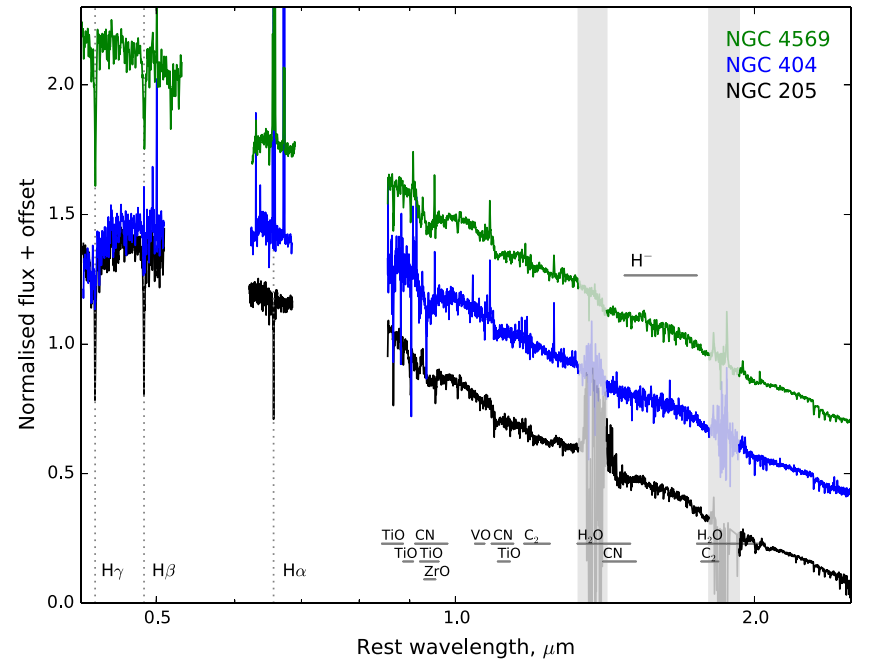

Figure 4. Optical-NIR spectra of "intermediate-age" galaxies (see text). The same molecular bands as shown in Figure 3 are indicated, as well as the " $H$ band bump" (stellar $\mathrm{H}^{-}$opacity minimum). Optical Balmer absorption lines are also indicated. Note that the wavelength coverage of the optical data (Ho et al. 1995) does not include the strong higher-order Balmer lines that are present in the Cid Fernandes et al. (2004) spectra of these nuclei. The NIR spectra were normalized around $0.9 \mu \mathrm{m}$ and offset for clarity. As the optical spectra were taken through a wider slit, they have been scaled in flux to match the extrapolation of a low-order polynomial fit to the IR spectra. Regions of poor atmospheric transmission, as defined in Figure 2, are shown in gray.

These bands are generally weak, broad, and often overlapping in wavelength, and stars other than TP-AGB stars may contribute to them. For instance, the noticeable absorption around $0.94 \mu \mathrm{m}$ in our spectra may be a blend of $\mathrm{ZrO}(\mathrm{M}$ giants, S stars), CN (C-N stars, supergiants), and TiO (late-M giants and dwarfs). Similarly, the broad, shallow dip between $\sim$ $1.32-1.5 \mu \mathrm{m}$ is likely caused by both $\mathrm{H}_{2} \mathrm{O}$ and $\mathrm{CN}$. The relatively isolated $\mathrm{VO}$ feature at $\lambda \sim 1.05 \mu \mathrm{m}$ is particularly strong in late-type M giants (Rayner et al. 2009).

A full determination of the dependence of these molecular features on the stellar populations of these galaxies requires accurate stellar population synthesis modeling, and is beyond the scope of this work. However, as a preliminary exploration, in Figures 4 and 5 we compare the IR and optical spectra of galaxies identified as "intermediate-age" and "old."

The intermediate-age category consists of three objects that are well-known for their strong Balmer absorption lines, indicating star formation occurring within the last $1.5 \mathrm{Gyr}$. These galaxies are NGC 205, NGC 404, and NGC 4569 (Keel 1996; Ho et al. 2003; Bouchard et al. 2010; Seth et al. 2010). Roughly $70 \%$ of the optical light of NGC 205 is emitted by stars with ages $\sim 0.1-1 \mathrm{Gyr}$ (Bica et al. 1990; González Delgado et al. 1999), and this galaxy is used by Cid Fernandes et al. (2004) as the intermediate-age template object in their optical stellar population synthesis work on lowluminosity AGNs. They find that NGC 404 and NGC 4569 are the galaxies in their sample with the most significant intermediate-age populations, with upwards of $70 \%$ of the $4020 \AA$ A emission accounted for by the NGC 205 template.

The old galaxies are those with ages $\gtrsim 5 \mathrm{Gyr}$, based on published analysis of their optical spectra. The central optical spectra and/or line indices of these objects are best described by stellar populations with ages of $\sim 5-11 \mathrm{Gyr}$ (see references in Appendix A). Many of the galaxies in this sample fit this criterion, so for clarity of presentation we restrict that category

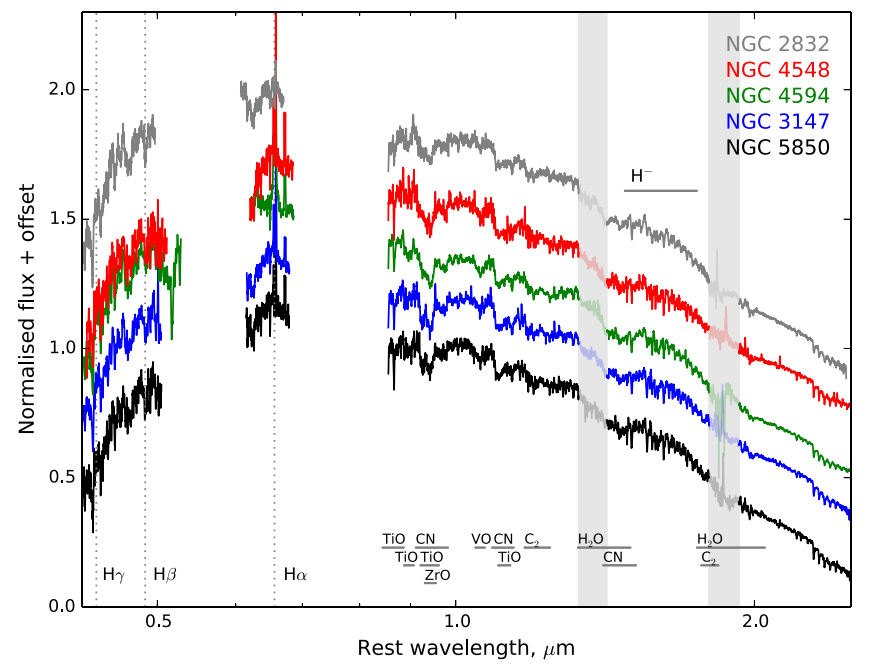

Figure 5. As for Figure 4, for "old" galaxies.

in Figure 5 to those with the cleanest telluric line removal (defined as $\mathrm{S} / \mathrm{N}>20$ at $1.32-1.37 \mu \mathrm{m}$ ). These are NGC 2768, NGC 2832, NGC 3147, NGC 4548, NGC 4594, and NGC 5850.

A qualitative comparison of Figures 4 and 5 suggests some systematic differences between the NIR spectra of intermediate-age and old galaxies. First, the NIR continuum shape of the old galaxies differs from that of the younger galaxies, becoming noticeably flatter at short wavelengths. This is not due to extinction; dust is not detected in Hubble Space Telescope (HST) optical images of NGC 2832, for example (Martel et al. 2004; Laine et al. 2003), and a variable nuclear UV source is detected in NGC 4594 (despite the galaxy being highly inclined; Maoz et al. 2005). Neither is AGN activity likely to be responsible for altering the shape of the spectra. NGC 205 and NGC 2832 have very different ages and continuum shapes, yet neither shows evidence for AGN activity (beyond the presence of a few, very weak emission lines in NGC 2832; Appendix A). The different spectral shape of the intermediate-age galaxies therefore appears to be due to their younger, bluer stellar populations relative to those of the old galaxies.

The $1.6 \mu \mathrm{m}$ "bump," which is strong in the atmospheres of K- and M-type giants and supergiants (Rayner et al. 2009), is also more pronounced in the old galaxies. Additionally, the broad, molecular absorption features are generally stronger in the old objects. This is noteworthy given that the stellar populations of the intermediate-age galaxies should contain large numbers of the TP-AGB stars discussed above and in Section 1. Depending on their age, metallicity, and pulsation cycles, these stars have deep features from molecules such as $\mathrm{CN}, \mathrm{C}_{2}, \mathrm{H}_{2} \mathrm{O}$, and VO (Lançon \& Mouhcine 2002). This is reflected by the evolutionary population synthesis models of Maraston (2005), which predict strong molecular features in stellar populations with ages $\sim 1 \mathrm{Gyr}$. However, the $1.1 \mu \mathrm{m} \mathrm{CN}$ band in the galaxies in Figure 4 is weak compared to that in the old galaxies, as are the $1.05 \mu \mathrm{m} \mathrm{VO}$ band and the feature at $\sim 0.93 \mu \mathrm{m}$ (likely a blend of $\mathrm{CN}, \mathrm{TiO}$, and $\mathrm{ZrO}$ ). Gauging the strength of the $\sim 1.4 \mu \mathrm{m} \mathrm{H}_{2} \mathrm{O}$ band is difficult because of the likely blending with $\mathrm{CN}$ absorption, the adjoining $\mathrm{H}^{-}$bump, and telluric residuals in NGC 205 and NGC 404, but any 

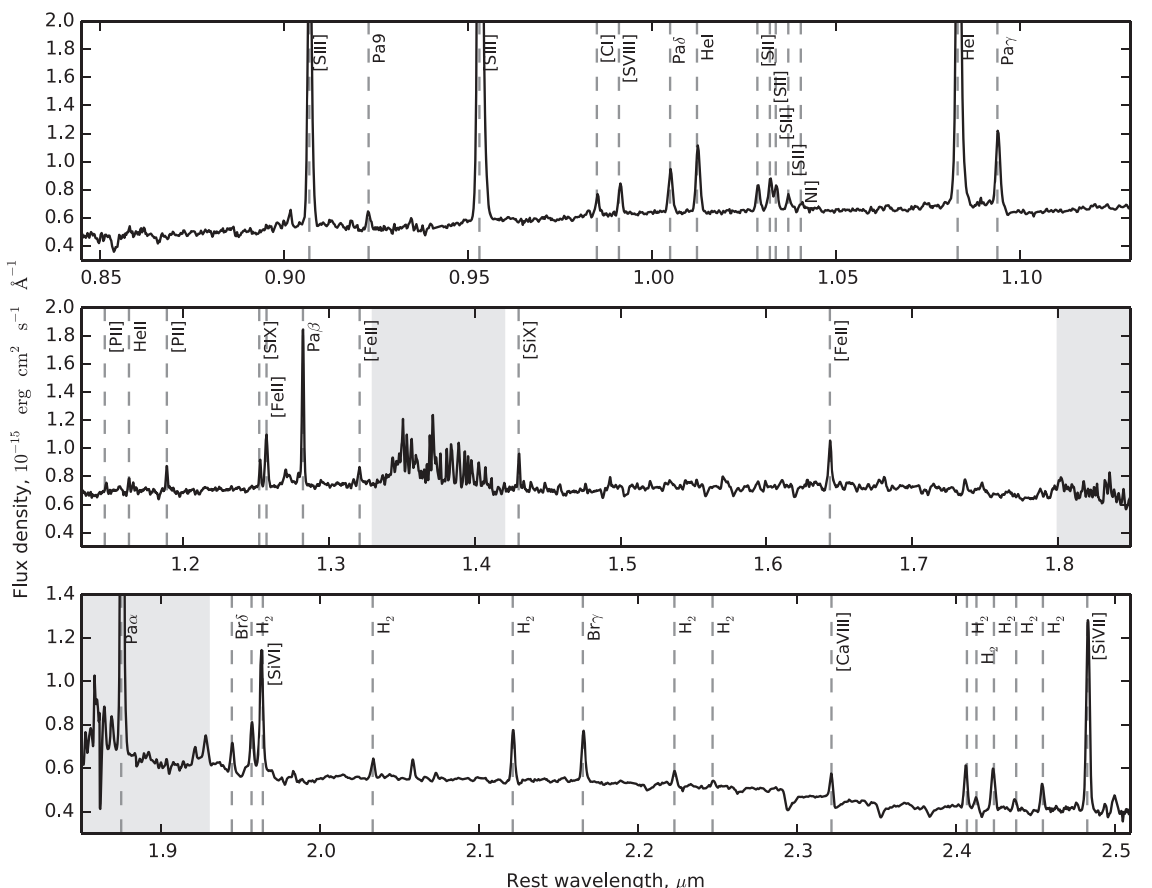

Figure 6. Spectrum of NGC 4388 (Sy 1.9), indicating the emission lines in the data. Regions of poor atmospheric transmission, as defined in Figure 2, are indicated in gray.

minimum around this wavelength appears no stronger in the intermediate-age galaxies than in the old ones.

This simple comparison, although based on just a few objects, suggests that models that predict a relatively featureless NIR spectrum will provide a better description of galaxies with significant intermediate-age populations. One caveat is that the intermediate-age galaxies all have relatively low stellar velocity dispersions $\left(20<\sigma_{*}<140 \mathrm{~km} \mathrm{~s}^{-1}\right)$, while with the exception of NGC $4548\left(\sigma_{*}=113 \mathrm{~km} \mathrm{~s}^{-1}\right)$, the old galaxies are high-dispersion systems with $140<\sigma_{*}<340 \mathrm{~km} \mathrm{~s}^{-1}$ (Ho et al. 2009; Riffel et al. 2015). The well-known relations between $\sigma_{*}$ and metallicity (Bender et al. 1993) and the abundances of carbon, nitrogen, and $\alpha$-elements (Schiavon 2007) then suggest that the intermediate-age galaxies are metal-poor, while the old galaxies are metal-rich. The spectral differences between these sets of galaxies may therefore reflect differences in metallicity as well as in age.

This conclusion also relies on the optical spectra being a reasonably reliable indicator of the age of the stellar population that dominates in the NIR. Differences in stellar populations derived from optical and NIR spectra have been observed in star-forming and Seyfert galaxies (Riffel et al. 2009; Martins et al. 2013b). These differences have been attributed to the inclusion of regions of star formation in the wider optical slit; the detection of dust-enshrouded stellar populations in the NIR; and the differing sensitivity of the optical and NIR regimes to stars of different ages (e.g., Riffel et al. 2011a). It has also been found that post-starburst spectra indicative of intermediate-age stars can arise in spatially isolated regions of a galaxy (Sanmartim et al. 2014). We are therefore working to obtain aperture-matched optical data for in-depth population synthesis of the galaxies in this sample. Nonetheless, these observations provide an initial illustration of the NIR characteristics of intermediate-age and old stellar populations in galaxies.

\subsection{Emission Lines}

Emission lines are also present in many of the spectra, being particularly numerous and prominent in some of the Seyferts. In Figure 6, the Seyfert 1.9 NGC 4388 is used to illustrate and identify many of these lines. Detailed and quantitative analysis of the line emission is being presented in a separate paper (O. González-Martín et al. 2015, in preparation), so here we make some general remarks about the detected lines and their potential utility.

Broadly speaking, the optical spectrum is a good predictor of the IR emission-line spectrum: objects with plentiful, strong lines in their optical spectrum have a rich IR line spectrum, and vice versa. The most commonly detected, and generally strongest, features are $[\mathrm{S}$ III $] \lambda \lambda 0.907,0.953$; He I $\lambda 1.083$; and $[\mathrm{Fe}$ II $] \lambda \lambda 1.257,1.644$ (all wavelengths in $\mu \mathrm{m}$ ). At least some of these lines are present in most of the Seyferts and type 1.9 LINERs alike, although they are not detected in several type 2 LINERs. Lower-intensity lines are also present in some of the spectra. $\left[\mathrm{C}_{\mathrm{I}}\right] \lambda 0.985,[\mathrm{~S} \mathrm{II}] \lambda 1.029,1.032$ (the redder line being a blend of three $\left[\mathrm{S}_{\mathrm{II}}\right]$ transitions), and $\left[\mathrm{N}_{\mathrm{I}}\right] \lambda 1.041$ are fairly common, while $\left[\mathrm{P}_{\mathrm{II}}\right] \lambda 1.147,1.188$, Не II $\lambda \lambda 1.012,1.163$, and $[\mathrm{Fe}$ II $] \lambda 1.321$ are detected in a small fraction of the Seyferts.

The most intense hydrogen recombination line in the nearinfrared range, $\mathrm{Pa} \alpha$, may be present in some spectra but its location in a region of very poor atmospheric transmission often makes its detection highly uncertain (see Appendix B). The next-most intense $\mathrm{H}$ recombination lines, $\mathrm{Br} \gamma$ and $\mathrm{Pa} \beta$, are also visible in a minority of the spectra, along with $\mathrm{Pa} \gamma$ (and higher-order $\mathrm{H}$ recombination lines) in a few. When detected, these lines and the $\mathrm{He}_{\mathrm{I}} \lambda 1.083$ recombination line show broad pedestals in the spectra of most of the Seyfert 1-1.9 (NGC 2639, NGC 3031, NGC 4235, NGC 4258, NGC 4395, NGC 4579, NGC 5033, and NGC 7469) and LINER 1.9 (NGC 315, NGC 1052, NGC 3998, NGC 4203, NGC 4450, and NGC 4750) objects. In most other cases the emission lines 
are too faint to distinguish any broad components, if present. On the other hand, almost none of the type 2 Seyferts or LINERs show broad components to the recombination lines.

Some of these emission lines can be used as tracers of the dominant ionization mechanisms. For example, strong [Fe II] emission is believed to be indicative of shock-excited gas, demonstrated by the fact that it occurs in the filaments of supernova remnants, in contrast to the weak [Fe II] emission characteristic of the photoionized gas in H II regions (Mouri et al. 1990; Rodríguez-Ardila et al. 2004). Several mechanisms can contribute to the $[\mathrm{Fe}$ II $]$ emission in galaxy nuclei: photoionization by extreme UV to soft X-ray radiation from the central source (Simpson et al. 1996), shocks induced by interaction of the radio jets with the surrounding medium, and shocks produced by supernova remnants present in starforming regions (Forbes \& Ward 1993; Alonso-Herrero et al. 2000).

The $[\mathrm{Fe} I \mathrm{II}] \lambda 1.257 / \mathrm{Pa} \beta$ line ratio (or, equivalently $[\mathrm{Fe}$ II $]$ $\lambda 1.644 / \mathrm{Br} \gamma$ ) has been widely used to investigate the origin of the $[\mathrm{Fe}$ II $]$ emission. These ratios increase from $\mathrm{H}_{\text {II }}$ regions (photoionization by hot stars) to supernova remnants (shock excitation), with starbursts and active galaxies showing intermediate values (Alonso-Herrero et al. 1997; RodríguezArdila et al. 2004). Galaxies exhibiting $[\mathrm{Fe}$ II $] \lambda 1.257 / \mathrm{Pa} \beta<$ 0.6 are usually classified as starbursts, those with $[\mathrm{Fe}$ II] $] \lambda 1.257 /$ $\mathrm{Pa} \beta>2$ as LINERs, and Seyfert galaxies usually have $0.6<$ $[\mathrm{Fe}$ II $] \lambda 1.257 / \mathrm{Pa} \beta<2$ (Larkin et al. 1998; Rodríguez-Ardila et al. 2004, 2005; Riffel et al. 2013a, although see Martins et al. 2013a for counterexamples).

High-ionization transitions (e.g., [S VIII $] \lambda 0.991$, [S Ix] $] \lambda 1.252$, $[\mathrm{Si} \mathrm{x}] \lambda 1.430, \quad[\mathrm{Si} \mathrm{VI}] \lambda 1.963$, and $[\mathrm{Ca} \mathrm{VIII}] \lambda 2.322)$ are also detected in a few of the Seyferts in this sample (NGC 2273, NGC 4388, NGC 4395, NGC 7469, and 1H1934-063). These "coronal" lines (ionization potential $>100 \mathrm{eV}$ ) can only exist very close to the ionization source, making them unique tracers of AGN activity and energetics. The simultaneous observation of very high- and low-ionization lines in AGN spectra implies a wide variety of physical conditions over the regions probed by the spectra, and the detection of strong coronal lines is indicative of the presence of extreme UV and X-ray photons (Prieto \& Viegas 2000). The association of strong coronal lines with soft, thermal X-ray emission suggests that photoionization by the central source is the dominant excitation mechanism for coronal line emission in AGNs (Rodríguez-Ardila et al. 2011).

Finally, emission from the $\mathrm{H}_{2}$ molecule is detected in around half of the galaxies. The strongest lines are from the $\mathrm{H}_{2} 1-0 \mathrm{~S}$ (3) $1.96 \mu \mathrm{m}$ and $\mathrm{H}_{2} 1-0 \mathrm{~S}(1) 2.12 \mu \mathrm{m}$ transitions, although lower intensity lines such as $\mathrm{H}_{2} 1-0 \mathrm{~S}(2) 2.03 \mu \mathrm{m}, \mathrm{H}_{2} 1-0 \mathrm{Q}(2)$ $2.41 \mu \mathrm{m}$, and $\mathrm{H}_{2} 1-0 \mathrm{Q}(3) 2.42 \mu \mathrm{m}$ are also detected in some of the spectra. There are two main $\mathrm{H}_{2}$ excitation mechanisms: "thermal" and "non-thermal" (Moorwood \& Oliva 1988; Rodríguez-Ardila et al. 2004, and references therein). In the thermal case, the molecules are heated by shocks, UV photons or X-rays, whereas in the non-thermal case the molecules fluoresce after being excited by absorption of a UV photon or collision with a fast electron from an X-ray ionized plasma. These two mechanisms produce different relative emission line intensities, which can be used to identify the dominant mechanism. In particular, the $\mathrm{H}_{2} 1-0 \mathrm{~S}(1) / 2-1 \mathrm{~S}(1)$ line ratio is generally higher for thermal excitation $(\sim 5-10)$ than for UV fluorescence $(\sim 1.82)$, as proposed by Mouri (1994). Alternatively, rotational and vibrational temperatures can be determined using the expressions given by Reunanen et al. (2002), which involve the $\mathrm{H}_{2} 1-0 \mathrm{~S}(1) / 2-1 \mathrm{~S}(1)$ and $\mathrm{H}_{2} 1-0 \mathrm{~S}$ $(2) / 1-0 S(0)$ line ratios. In the case of thermal excitation, both temperatures are similar, whereas in the case of fluorescent excitation, the vibrational temperature is larger.

\section{CONCLUSIONS}

We have presented moderate-resolution $(R \sim 1300-1800)$ NIR spectra of the nuclear regions of 50 nearby $(D=1-92 \mathrm{Mpc})$ galaxies, with complete spectral coverage from $\sim 0.85-2.5 \mu \mathrm{m}$. The galaxies span a variety of morphological types, and host comparable numbers of Seyfert and LINER nuclei (as well as a handful of TOs and inactive nuclei). This, together with the wide wavelength coverage and good S/N of the spectra, establishes the NIR spectral properties of galaxies hosting a range of nuclear activity and stellar populations. The emission lines detected in many of the spectra may be used to examine the ionization mechanisms at work in the nuclei, while the underlying absorption line spectra are potentially of use to studies of the IMF and interesting stages of stellar evolution. We are using the data to investigate a range of issues related to low-luminosity AGNs and their host galaxies, and we encourage interested readers to explore whether the spectra-now available to the public - may be of use to their own research as well.

R.E.M. is grateful to NRC-Herzberg Institute of Astrophysics for their hospitality during a portion of this work, and would like to acknowledge Else Starkenburg and Tom Geballe for helpful discussions. We also thank the anonymous referee for a timely report that helped improve the paper. Supported by the Gemini Observatory, operated by the Association of Universities for Research in Astronomy, Inc., on behalf of the international Gemini partnership of Argentina, Australia, Brazil, Canada, Chile, and the USA. C.R.A. is supported by a Marie Curie Intra European Fellowship within the 7th European Community Framework Programme (PIEF-GA2012-327934). L.C.H. acknowledges support from the Kavli Foundation, Peking University, and the Chinese Academy of Science through grant No. XDB09030100 (Emergence of Cosmological Structures) from the Strategic Priority Research Program. A.A.-H. acknowledges support from the Spanish Plan Nacional de Astronomía y Astrofísica under grant AYA201231447. A.R.A. thanks the Conselho Nacional de Desenvolvimento Científico e Tecnológico $(\mathrm{CNPq})$ for partial support of this work through grant 307403/2012-2. This research used the facilities of the Canadian Astronomy Data Centre operated by the National Research Council of Canada with the support of the Canadian Space Agency.

\section{APPENDIX A SPECTRA AND NOTES FOR INDIVIDUAL GALAXIES}

In this Appendix we present the optical and near-IR spectrum of each object, along with brief descriptions of the galaxies. The optical data are from Ho et al. (1995). For ease of comparison, the spectra in the top panels of Figures A1 -A1.48 are all shown on a scale of $0.1-2.5 \times$ the median $H$-band flux.

To aid the intercomparison of the spectra, a "reference" set of emission line wavelengths has been indicated on each one. These lines are given in Table A1. They consist mainly of those lines that are most frequently detected, and include the 
five brightest $\mathrm{H}_{2}$ lines in the $K$ band. The set also contains two lines, $\mathrm{Pa} \beta$ and $\mathrm{Br} \gamma$, which are not detected in most objects but which have been of interest for stellar population and kinematic studies of active galaxies (e.g., Davies et al. 2007; Riffel et al. 2011b). For the Seyfert galaxies with many emission lines (NGC 4395, NGC 4388, NGC 2273, $1 \mathrm{H}$ 1934-063, NGC 7469), some additional lines are also indicated.

\section{A.1 Seyfert 1-1.9}

$N G C 4235$ (S1.2)

The center of this nearly edge-on galaxy in the Virgo Cluster is bisected by a dust lane. The nucleus displays an emission line spectrum characteristic of type 1 Seyfert galaxies, although optical spectroscopy shows that the broad $\mathrm{H} \alpha$ line has a peculiar secondary hump redward of $[\mathrm{N}$ II]. The NLR appears compact with a possible extension in [O III] toward the northeast with a PA $\sim 48^{\circ}$ and a length of $\sim 4.4$ or $\sim 0.9 \mathrm{kpc}$ (Pogge 1988).

The GNIRS nuclear spectrum (Figure A1) shows that NGC 4235 contains very broad permitted He I lines, the He I $1.083 \mu \mathrm{m}$ line being the brightest line in the observed wavelength interval. The broad $\operatorname{Pa} \beta$ (and possibly also $\operatorname{Pa} \delta$ ) line has a complex profile with a prominent blue peak and a very broad, weak red wing centered about $7700 \mathrm{~km} \mathrm{~s}^{-1}$ from the systemic velocity. This value is significantly higher than the shift of $\sim 5400 \mathrm{~km} \mathrm{~s}^{-1}$ found in the red peak of the $\mathrm{H} \alpha$ line measured from the SDSS optical spectrum, possibly indicating that this AGN is highly variable. Forbidden lines of $[\mathrm{S} \mathrm{III]} \lambda \lambda$ $0.907,0.953 \mu \mathrm{m}$, and [Fe II $] 1.257 \mu \mathrm{m}$ are also identified in the spectrum.

The continuum shows prominent stellar absorption features, from the very blue edge, where the $\mathrm{CaT}$ is strong by the standards of a Seyfert nucleus, to the red end, where deep CO absorption bands are observed. The $H$ band displays a broad bump which is interpreted as a minimum in $\mathrm{H}^{-}$opacity in cool stars in the galaxy (Rayner et al. 2009). Peletier et al. (2007) find the central stellar population of NGC 4235 to be old, although they note that AGN emission makes the derived age rather uncertain. $K$-band observations of this source have been published by Ivanov et al. (2000) and Imanishi \& Wada (2004), but to the best of our knowledge this is the first spectroscopy of the other NIR bands.

NGC 3031 (S1.5)

Also known as M81, this spiral galaxy is part of an interacting galaxy group that also includes M82 and NGC 3077 (Yun et al. 1994). Although the active nucleus is classified as a low-luminosity Seyfert 1.5 by Ho et al. (1997a), it is often referred to as a LINER, and Ho et al. (1996) note that its ionization parameter is low relative to typical Seyferts. The nucleus appears unresolved in HST optical images (Devereux et al. 1997). At radio wavelengths, NGC 3031 shows a compact morphology and nuclear variability (Ho et al. 1999, and references therein), and at high angular resolution the infrared/optical SED is flatter than that of typical Seyfert templates (Mason et al. 2012). Based on multicolor photometry, Kong et al. (2000) find the central stellar population of NGC 3031 to be old (>8 Gyr), and that the outer regions of the galaxy are significantly older. This is in agreement with optical spectroscopy analyzed by Boisson et al. (2000). The nearinfrared spectrum presented here (Figure A1.2) shows a prominent $\mathrm{He}_{\mathrm{I}} 1.083 \mu \mathrm{m}$ line with a broad pedestal, and possibly broad $\mathrm{Pa} \beta$ as well. The $[\mathrm{Fe}$ II $] 1.257 \mu \mathrm{m}$ line detected by Alonso-Herrero et al. (2000) is also present, along with several other forbidden transitions including $\left[\mathrm{S} \mathrm{III}_{,},[\mathrm{S} \mathrm{II}]\right.$, and $\left[\mathrm{N}_{\mathrm{I}}\right]$.

NGC 5033 (S1.5)

This highly inclined spiral galaxy forms a pair with NGC 5005 (Helou et al. 1982). It hosts a Seyfert 1.5 nucleus, obscured by strong spiral dust lanes revealed by HST/NICMOS and WFPC2 images (Martini et al. 2003; Erwin 2004). The galaxy nucleus is conspicuously brighter in the near-infrared than in the optical, confirming the presence of nuclear dust lanes that are probably responsible for the flattening of the continuum at short wavelengths in the GNIRS spectrum (Figure A1.3; cf. NGC 3031, for example). High internal reddening was also noted by Boisson et al. (2000), who find that the central stellar population of NGC 5033 is old, with just a few percent of the $5450 \AA$ emission coming from A-type stars. The optical emission-line spectrum of this galaxy has characteristics of both LINERs and Seyfert galaxies, and the broad $\mathrm{H} \alpha$ emission is variable (Ho et al. 1995). The nearinfrared spectrum is fairly typical of a Seyfert nucleus, with He I lines showing broad pedestals and lines of $[\mathrm{S} \mathrm{III}],[\mathrm{Fe} \mathrm{II}]$, and $\left[\mathrm{C}_{\mathrm{I}}\right]$ detected, as well as several $\mathrm{H}_{2}$ emission lines in the $K$ band (also noted by Ivanov et al. 2000 and Bendo \& Joseph 2004).

NGC 4395 (S1.8)

This galaxy (Figure A1.4) holds several records: it is the nearest and faintest Seyfert 1 nucleus (Filippenko \& Sargent 1989), and it is one of the intrinsically weakest nuclear X-ray sources observed to date (Lira et al. 1999; Moran et al. 1999). NGC 4395 is also highly unusual as an AGN host because of its late Hubble type: it is classified as a Magellanic spiral (Sm; Ho et al. 2001). Last but not least, it harbors one of the smallest supermassive BHs with an accurately determined mass $\left(3 \times 10^{5} M_{\odot}\right.$ Peterson et al. 2005). With all these superlatives, it is not surprising that the radio, IR, optical, and X-ray characteristics of this AGN have been extensively discussed by several authors (e.g., Lira et al. 1999; Iwasawa et al. 2000; Moran et al. 2005; Peterson et al. 2005; Skelton et al. 2005; Minezaki et al. 2006; Wrobel \& Ho 2006). Curiously, very few spectroscopic observations in the NIR are found in the literature. Kraemer et al. (1999), for instance, reported optical/NIR spectroscopy from the atmospheric cutoff (3200 $\AA$ ) to almost $1 \mu \mathrm{m}$, along with $L$-band spectroscopy of this object. To the best of our knowledge, the GNIRS spectrum shown here is the first $0.85-2.5 \mu \mathrm{m}$ spectrum in the literature. It is dominated by nebular lines, both permitted and forbidden, on top of a weak power-law continuum with very little sign of the underlying stellar population. $\left[\mathrm{S}_{\mathrm{III}}\right] 0.907,0.953 \mu \mathrm{m}, \mathrm{He}_{\mathrm{I}}$ $1.083 \mu \mathrm{m}$, and the Paschen series of $\mathrm{HI}$ are, by far, the brightest emission lines in the spectrum. Broad permitted lines of $\mathrm{H}$ I, He I, Fe II, and O I, typical of the Seyfert 1 nature of this object, are present, and weak, high-ionization lines of [S VIII], [S IX], [Si vI], [Si vII], and [Si x] are also detected. Molecular hydrogen lines are bright in the $K$-band spectrum of this source.

NGC 2639 (S1.9)

Like NGC 4258, NGC 2639 hosts a rare nuclear $\mathrm{H}_{2} \mathrm{O}$ megamaser, interpreted as arising in a dense accretion disk (Braatz et al. 1994; Wilson et al. 1995). The stellar velocity structure of the nucleus is also noteworthy, with measurements of the calcium triplet lines indicating a drop in velocity dispersion toward the nucleus that may be due to the presence of a young stellar population in a circumnuclear disk (Márquez 


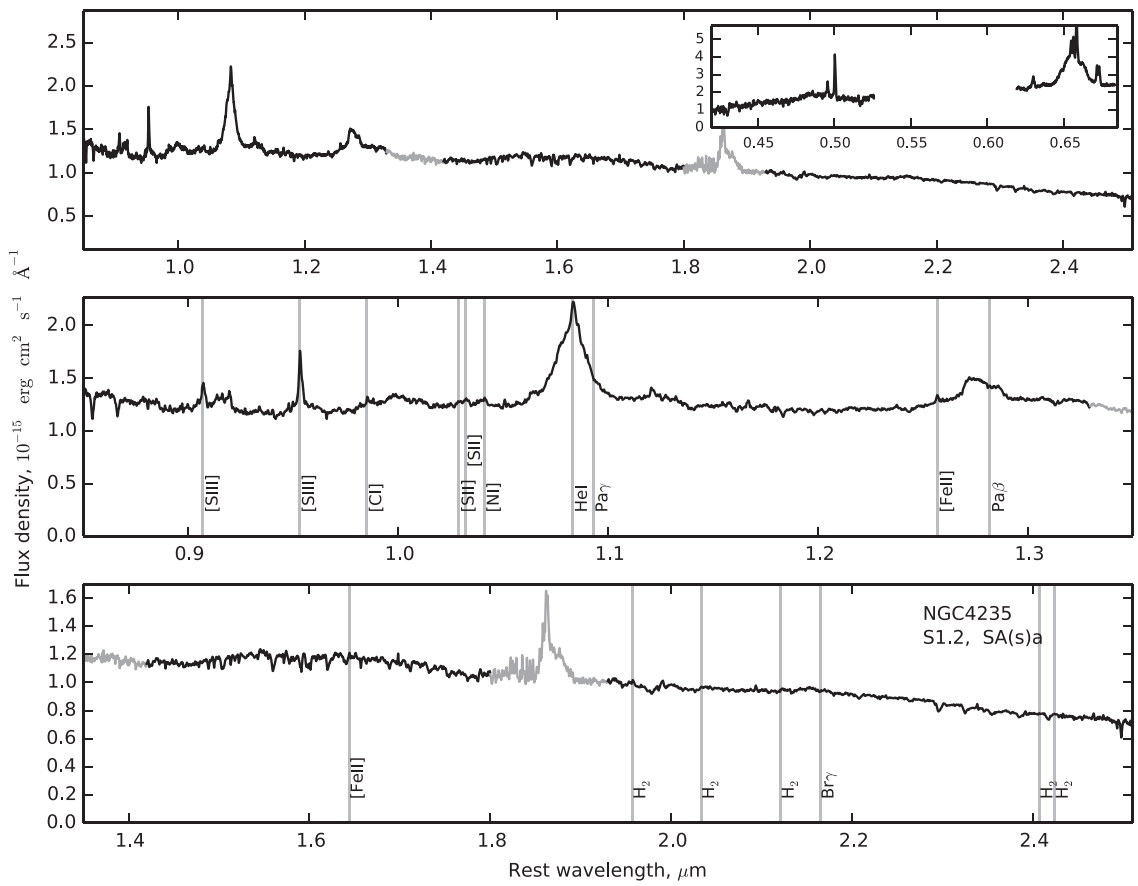

Figure A1. NIR spectrum of NGC 4235, along with the optical spectrum of Ho et al. (1995, inset). Regions of very poor atmospheric transmission $(\lesssim 20 \%$ for 3 mm $\mathrm{H}_{2} \mathrm{O}$ at zenith) are plotted in gray. Vertical lines indicate the set of emission lines given in Table A1. For absorption line identifications, see Figure 3.

(The complete figure set (49 images) is available.)

et al. 2003). The IR spectrum of NGC 2639 (Figure A1.5) is rather unremarkable, with only a handful of forbidden and permitted emission lines detected, along with some $\mathrm{H}_{2}$ emission in the $K$ band. The [Fe II] emission in the $J$ band was also noted by Alonso-Herrero et al. (2000). Stellar absorption features similar to those shown in Figure 3 are visible throughout the spectrum.

NGC 4258 (S1.9)

NGC 4258 is well-known for its nuclear $\mathrm{H}_{2} \mathrm{O}$ megamaser, which traces a dense, edge-on disk on sub-parcsec scales (Watson \& Wallin 1994; Greenhill et al. 1995; Miyoshi et al. 1995). As such, it has been the target of numerous distance measurements using several different techniques (Macri et al. 2006; Mager et al. 2008; Humphreys et al. 2013). The nucleus of NGC 4258 also contains a variable, relativistic radio jet (Doi et al. 2013), which may, on larger scales, be interacting with molecular clouds in the galaxy's disk (Krause et al. 2007). Overall, the infrared spectrum (Figure A1.6) rather closely resembles that of NGC 2639 (Figure A1.5), another megamaser source, although the stellar absorption features appear somewhat deeper in NGC 4258. Differences in the emission lines are also apparent, such as stronger [S III] lines in NGC 4258 than in NGC 2639, and weaker or undetected $\mathrm{H}_{2}$ and [Fe II] emission. It appears that no optical stellar population synthesis has been published for this galaxy.

\section{NGC 4388 (S1.9)}

Classified as a Seyfert 2 galaxy by Phillips \& Malin (1982) and Seyfert 1.9 by Ho et al. (1997a), NGC 4388 (Figure 6, A1.7) is one of the first galaxies in which a conically shaped NLR was detected (Pogge 1988). This AGN, which is host to an $\mathrm{H}_{2} \mathrm{O}$ megamaser (Kuo et al. 2011), lies in an $\mathrm{SB}(\mathrm{r})$ a galaxy close to edge-on. For this reason, it has an extremely chaotic appearance at small scales, in part due to a host galaxy dust lane passing to the immediate north of the nucleus. Its continuum emission reflects the dusty nature of this source, being heavily absorbed from $1.4 \mu \mathrm{m}$ bluewards. NGC 4388 has been studied extensively in various wavelength regimes, including the NIR (see references in Table 2). The GNIRS spectrum reveals a wealth of emission lines, many of them never reported in the literature. In particular, spectral features located in the region $1-1.15 \mu \mathrm{m}$ and redwards of $2.36 \mu \mathrm{m}$ are new detections. Emission lines of $[\mathrm{S}$ III $] 0.907,0.953 \mu \mathrm{m}$, He I $1.083 \mu \mathrm{m}$, and the Paschen series of $\mathrm{H}_{\mathrm{I}}$ are the brightest ones in the $0.84-2.5 \mu \mathrm{m}$ interval. The strong high-ionization spectrum of NGC 4388 is also noteworthy, with [Si vII] $2.483 \mu \mathrm{m}$, for instance, being the second brightest line in the $K$ band after Pa $\alpha$. Moreover, [S VIII], [Si VII], and [Si x] were not previously reported in the literature, as well as the low-ionization forbidden emission of $\left[\mathrm{C}_{\mathrm{I}}\right],\left[\mathrm{S}_{\mathrm{II}}\right],\left[\mathrm{N}_{\mathrm{I}}\right]$, and $\left[\mathrm{P}_{\mathrm{II}}\right]$, which are also prominent in the spectrum. Most of the above emission lines extend across the slit, from NE to SW. The CaT and $\mathrm{CO}$ absorption bands are visible at the blue and red edges of the spectrum, respectively, as is $\mathrm{CN}$ absorption at $1.1 \mu \mathrm{m}$. The analysis of the nuclear and extended emission of this object is the subject of a separate publication (A. RodríguezArdila et al. 2015, in preparation).

\section{$N G C 4565$ (S1.9)}

NGC 4565 is an SAb galaxy that is highly inclined to the line of sight. Proctor et al. (2000) present evidence for a kinematically cold population at the center of NGC 4565 that may be associated with ongoing star formation. As might be expected from the relatively weak emission lines in the optical spectrum of NGC 4565, the GNIRS spectrum (Figure A1.8) of this object contains just a few, weak lines from [S III], [C I $], \mathrm{He}_{\mathrm{I}}$, and [Fe II]. $\mathrm{H}_{2}$ emission has been detected at 17 and $28 \mu \mathrm{m}$ (Laine et al. 2010), but molecular hydrogen lines are not seen in Figure A1.8, or in the $K$-band spectrum presented by Doyon et al. (1989). Absorption lines from the nuclear stellar 
Table A1

The "Reference" Set of Emission Lines Shown in Figures A1-A1.49

\begin{tabular}{|c|c|}
\hline Line & Wavelength, $\mu \mathrm{m}$ \\
\hline$[\mathrm{S}$ III] $]$ & 0.907 \\
\hline$[\mathrm{S}$ III] $]$ & 0.953 \\
\hline$\left[\begin{array}{ll}\mathrm{C}_{1}\end{array}\right]$ & 0.985 \\
\hline$\left[\mathrm{S}_{\mathrm{III}}\right]$ & 1.029 \\
\hline$[\mathrm{S}$ III] & 1.032 \\
\hline$\left[\mathrm{N}_{\mathrm{I}}\right]$ & 1.041 \\
\hline $\mathrm{He}_{\mathrm{I}}$ & 1.083 \\
\hline $\mathrm{Pa} \beta$ & 1.282 \\
\hline $\operatorname{Pa} \gamma$ & 1.093 \\
\hline$[\mathrm{Fe}$ II $]$ & 1.257 \\
\hline$[\mathrm{Fe} \mathrm{II}]$ & 1.644 \\
\hline $\mathrm{H}_{2}$ 1-0 S(3) & 1.958 \\
\hline $\mathrm{H}_{2} 1-0 \mathrm{~S}(2)$ & 2.034 \\
\hline $\mathrm{H}_{2} 1-0 \mathrm{~S}(0)$ & 2.122 \\
\hline $\mathrm{Br} \gamma$ & 2.165 \\
\hline $\mathrm{H}_{2} 1-0 \mathrm{Q}(1)$ & 2.407 \\
\hline $\mathrm{H}_{2} 1-0 \mathrm{Q}(3)$ & 2.424 \\
\hline
\end{tabular}

population, including the CaT, $\mathrm{CO}$ band heads, and a slew of features in the $H$ band, are clearly visible in the spectrum.

NGC 4579 (S1.9/L1.9)

NGC 4579 contains a low-luminosity AGN that may be accreting via an advection-dominated accretion flow (Quataert et al. 1999; Yu et al. 2011). Mapping of the gravitational torques in the galaxy suggest that material is being driven to within 50 pc of the AGN (García-Burillo et al. 2009). Palacios et al. (1997) present evidence based on optical and NIR Mg indices that the stellar population in NGC 4579 may be fairly young, with a characteristic age of roughly $2.5 \mathrm{Gyr}$. Absorption lines from the nuclear stellar population in NGC 4579 are clearly visible in the GNIRS spectrum (Figure A1.9), as are emission lines of $[\mathrm{S} I I I],\left[\mathrm{CI}_{\mathrm{I}}\right], \mathrm{He}_{\mathrm{I}},\left[\mathrm{Fe}_{\mathrm{II}}\right]$, and $\mathrm{H}_{2}$ that are common in this class of objects. The [Fe II $1.26 \mu \mathrm{m}$ line was previously reported by Alonso-Herrero et al. (2000), and the morphology and kinematics of the $K$-band $\mathrm{H}_{2}$ emission have been analyzed by Mazzalay et al. (2013, 2014).

\section{A.2 Seyfert 2}

$N G C 1167$ (S2)

This lenticular galaxy resides in a rich environment, with several companions within a projected distance of $350 \mathrm{kpc}$ (Struve et al. 2010). The galaxy hosts an active nucleus with sub-parsec scale jets (Nagar et al. 2005; Giovannini et al. 2001), suspected to be obscured by a Compton-thick column of material (Panessa et al. 2006; Akylas \& Georgantopoulos 2009). The stellar population within the half-light radius is fairly old, with a luminosity-weighted mean age of $~ 6 \mathrm{Gyr}$ (González Delgado et al. 2014). The GNIRS spectrum of this object (Figure A1.10) exhibits a fairly sparse set of emission lines, from [S III], HeI, [Fe II], and $\mathrm{H}_{2}$.

NGC 1358 (S2)

The nucleus of this barred galaxy shows irregular dusty structures (Malkan et al. 1998). Storchi-Bergmann et al. (1998) find that $64 \%$ of the nuclear optical emission of NGC 1358 can be accounted for by a $10 \mathrm{Gyr}$ template, with almost all of the remainder from a 1 Gyr template. On the other hand, Pérez \& Sánchez-Blázquez (2011) estimate the age of the central stellar population to be in the region of $6 \mathrm{Gyr}$. The NIR spectrum of this object (Figure A1.11) bears a broad resemblance to that of NGC 1167, containing only a handful of weak emission lines. The $\sim 0.93 \mu \mathrm{m}$ absorption, likely a blend of $\mathrm{ZrO}, \mathrm{CN}$, and $\mathrm{TiO}$ (Section 4.2), appears to be unusually deep in this object.

NGC 2273 (S2)

This galaxy, classified as Seyfert 2 by Ho et al. (1997a), has been extensively studied in the literature, from radio to X-ray wavelengths (Terashima et al. 2002; Anderson \& Ulvestad 2005; Falcón-Barroso et al. 2006). It is a well-known barred galaxy (Ferruit et al. 2000), with nuclear spiral arms inside a ringlike dust feature (Laurikainen et al. 2005). Very Long Baseline Array (VLBA) imaging shows an elongated multiplecomponent structure aligned east-west on the sky (Anderson \& Ulvestad 2005). The brightest [O III] $\lambda 5007$ emission is a linear, jetlike structure extending $2^{\prime \prime}$ and aligned with the radio components. Fainter $[\mathrm{O} \mathrm{III}]$ emission is also present north and south of the nucleus. The GNIRS spectrum (Figure 1.12) was taken at a PA of $169^{\circ}$, that is, nearly perpendicular to the radio and most prominent $[\mathrm{O} \mathrm{III}] \lambda 5007$ emission. It is dominated by strong forbidden emission of [S III] $0.953 \mu \mathrm{m}$ and permitted He I at $1.083 \mu \mathrm{m}$. High ionization lines of [S VIII], [S IX], [Si vI], $[\mathrm{Si} \mathrm{VII}]$, and $[\mathrm{Si} \mathrm{x}]$ are also detected as well as low-ionization lines of $\left[\mathrm{Fe}_{\mathrm{II}}\right],\left[\mathrm{S}_{\mathrm{II}}\right],\left[\mathrm{N}_{\mathrm{I}}\right]$, and $\left[\mathrm{C}_{\mathrm{I}}\right]$. In the $K$ band, the nuclear spectrum includes numerous $\mathrm{H}_{2}$ lines. The shape of the continuum emission indicates that it is mostly starlight, with prominent $\mathrm{CaT}$ and $\mathrm{CO}$ absorption bands at the blue and red edges of the spectrum, respectively. In the $H$ band, absorption lines of $\mathrm{CO}, \mathrm{Si}, \mathrm{Mg}$, and $\mathrm{Fe}$ are also observed. Peletier et al. (2007) find that the strength of the $\mathrm{H} \beta$ line in the center of this galaxy indicates a significant $\sim 1$ Gyr stellar population.

NGC 2655 (S2)

NGC 2655 is a lenticular galaxy in a loose grouping of galaxies. Emission-line gas extends over several (Keel \& Hummel 1988), and the galaxy's diffuse, extended optical structure and complex $\mathrm{H}_{\mathrm{I}}$ dynamics have been interpreted as indicating a succession of mergers (Sparke et al. 2008). Optical line indices suggest recent star formation activity in this galaxy, and Sil'chenko \& Afanasiev (2006) find a luminosity-weighted mean age of 2 Gyr for the nucleus of NGC 2655. The NIR spectrum (Figure A1.13) shows several fairly weak, narrow emission lines, including several $\mathrm{H}_{2}$ transitions in the $K$ band. NGC 3079 (S2)

This edge-on spiral galaxy is known for its nuclear $\mathrm{H}_{2} \mathrm{O}$ megamaser (Henkel et al. 1984). It also contains a powerful outflow that appears to be influencing its satellite galaxy, NGC 3073 (Irwin et al. 1987; Filippenko \& Sargent 1992). The GNIRS spectrum of NGC 3079 (Figure A1.14) is characterized by a curved continuum, likely due to the high extinction toward the nucleus (Israel et al. 1998). $\mathrm{H}_{2}$ emission lines are relatively strong in this object, and Hawarden et al. (1995) argue that they arise in material excited by shocks in the nuclear outflow. The stellar absorption lines in the $H$ and $K$ bands broadly resemble those observed in many of the other galaxies examined here (although the $\mathrm{CO}$ bandheads are rather strong), but the $1.1 \mu \mathrm{m} \mathrm{CN}$ absorption appears somewhat weaker than usual.

\section{NGC 3147 (S2)}

NGC 3147 is a promising candidate for a "true" type 2 Seyfert nucleus, in which the BLR is genuinely lacking (rather than simply obscured; Bianchi et al. 2008; Matt et al. 2012). The optical spectrum of the source is well fit by a stellar population model with age $=8$ Gyr (Bruzual \& Charlot 1993). 


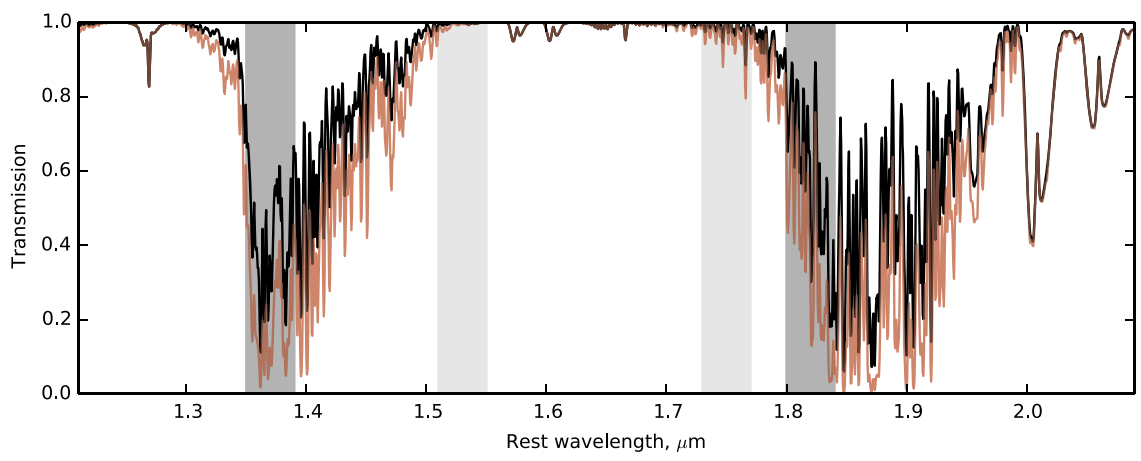

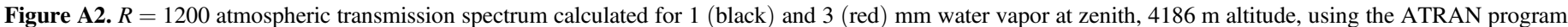

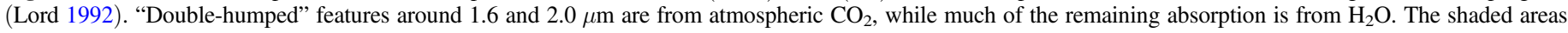
indicate the regions used to measure the S/N plotted in Figure B2.

The GNIRS spectrum (Figure A1.15) shows only a few, weak emission lines, along with many atomic and molecular stellar absorption lines. The good telluric line removal in the regions between the NIR atmospheric windows provides an opportunity to study in detail the stellar population properties of this source.

NGC 4725 (S2:)

NGC 4725 is a double-barred galaxy in which a handful of supernovae have occurred in recent decades. The optical classification of NGC 4725 as a Seyfert 2 is somewhat uncertain (Ho et al. 1997a) and although a hard X-ray source is detected at the nucleus, its luminosity is comparable to that of surrounding, off-nuclear sources (Ho et al. 2001). Although younger than the outer regions of the galaxy, the central stellar population is fairly old, with a luminosity-weighted mean age in the region of 4-5 Gyr (de Lorenzo-Cáceres et al. 2013). No emission lines are detected in the GNIRS spectrum (Figure A1.16), although numerous stellar absorption lines, from the $K$-band $\mathrm{CO}$ bandheads and $0.85 \mu \mathrm{m} \mathrm{CaT}$ to the plethora of $\mathrm{CO}$ and metallic lines in the $H$ band, are visible. As with NGC 3147, the good telluric line removal means that the $\mathrm{S} / \mathrm{N}$ in the spectrum is good at the locations of the $\mathrm{H}_{2} \mathrm{O}$ and $\mathrm{C}_{2}$ bands that are important for testing NIR stellar population models (Section 4.2).

NGC 5194 (S2)

NGC 5194, or M51, is a face-on spiral galaxy that is interacting with a neighboring system, NGC 5195 (e.g., Smith et al. 2010). $\mathrm{H}_{2} \mathrm{O}$ maser activity is detected in this galaxy, although it is unclear whether it is associated with an obscuring torus, jet, or star-forming region (Ho et al. 1987; Hagiwara et al. 2001; Hagiwara 2007). The stellar population in the inner few hundred parsecs of the galaxy is generally old, but this underlying distribution is punctuated by groups of massive stars (Lamers et al. 2002). Lines of [S III] are the strongest in the GNIRS spectrum (Figure A1.17), but emission from $\mathrm{He}$, [Fe $\mathrm{II}]$ and $\mathrm{H}_{2}$ is also fairly prominent. Despite the line emission, many stellar absorption features are clearly visible in the spectrum.

$N G C 7743$ (S2)

NGC 7743 is a barred lenticular galaxy whose active nucleus appears to be highly obscured (González-Martín et al. 2009a). Raimann et al. (2001) find that the nuclear optical light is emitted by three stellar components, $\sim 40 \%$ each from an old, metal-rich and a 1 Gyr component, with the remaining $20 \%$ from a $100 \mathrm{Myr}$ population. According to Sil'chenko \& Afanasiev (2006), the luminosity-weighted mean age is
$<2$ Gyr. The IR emission-line spectrum (Figure A1.18) is fairly similar to that of NGC 2655, another Seyfert 2 object, and a number of $\mathrm{H}_{2}$ lines are present. $\mathrm{Br} \gamma$ is detected in the spectrum presented by Mould et al. (2012), taken through a $1^{\prime \prime}$ slit, but is not visible in that of (Hicks et al. 2013, $8 \times 8^{\prime \prime}$ aperture) or in Figure A1.18 (0.3 slit), suggesting spatial structure in that line.

\section{A.3 LINER 1.9}

NGC 266 (L1.9)

This LINER is hosted in strongly barred galaxy (VéronCetty \& Véron 2006) which has undergone an interaction with neighboring UGC 499 (Noordermeer et al. 2005). NGC 266 has been studied from X-rays (Younes et al. 2012) to radio frequencies (Nagar et al. 2005). In its optical spectrum, a broad $\mathrm{H} \alpha$ component is obvious even before profile decomposition, with a FWHM of the broad component of $1350 \mathrm{~km} \mathrm{~s}^{-1}$ (Ho et al. 1997a). With the possible exception of a weak, broad He I $1.08 \mu \mathrm{m}$ feature, no emission lines are detected in the GNIRS spectrum (Figure A1.19). Numerous stellar absorption lines, however, are visible in the spectrum. The nuclear stellar population of NGC 266 is found to be predominantly old, with just a $5 \%$ contribution of intermediate-age stars to the optical light (Cid Fernandes et al. 2004).

NGC 315 (L1.9)

The host galaxy of NGC 315 is a giant $\mathrm{cD}$ radio galaxy located in the Zwicky cluster 0107.5 + 3212 (Zwicky et al. 1996). Two-sided, well-resolved radio jets are shown in both VLA and VLBI observations (Cotton et al. 1999; Venturi et al. 1993). Nagar et al. (2005) reported an unresolved core in addition to the radio jet at VLBI resolutions. The high spatial resolution provided by Chandra imaging allowed the detection of X-ray jets, the most striking being the one extending $~$ $10^{\prime \prime}$ to the NW (Donato et al. 2004; González-Martín et al. 2009b). This elliptical galaxy has a nuclear dust disk and an unresolved optical nucleus (Capetti et al. 2000). At optical wavelengths the emission-line spectrum of NGC 315 has LINER characteristics (Ho et al. 1995), and the detection of a broad component to $\mathrm{H} \alpha$ (Ho et al. 1997a) supports the identification of this object as a LLAGN. The GNIRS spectrum (Figure A1.20) shows prominent CaT and CO absorption lines. Low ionization lines of $\left[\mathrm{S}_{\mathrm{III}}\right],\left[\mathrm{C}_{\mathrm{I}}\right]$, and $\left[\mathrm{Fe}_{\mathrm{II}}\right]$ are detected, together with $\mathrm{He}_{\mathrm{I}} 1.083 \mu \mathrm{m}$, and a hint of a broad Pa $\gamma$ emission line. The nuclear spectrum also includes at least one weak $\mathrm{H}_{2}$ line in the $K$ band. As with NGC 266, the stellar population of NGC 315 is old, with intermediate-age stars contributing only 
$10 \%$ of the optical light (Cid Fernandes et al. 2004, ; see also Zhang et al. 2008).

NGC 1052 (L1.9)

NGC 1052 is the brightest member of a small group which, together with the NGC 1069 group, makes up the Cetus I cluster (Wiklind et al. 1995). Broad lines are clearly detected in the optical spectrum (Barth et al. 1999; Ho et al. 1997a; Balmaverde \& Capetti 2014). NGC 1052 also shows $\mathrm{H}_{2} \mathrm{O}$ megamaser emission (Claussen et al. 1998), and variability in the radio, (Vermeulen et al. 2003), ultraviolet (Maoz et al. 2005), and X-rays (Hernández-García et al. 2013).The $\mathrm{X}$-ray data clearly indicate the presence of an unresolved nuclear source in the hard bands (Satyapal et al. 2005; González-Martín et al. 2006), and Gonzalez-Martin et al. (2014) recently used artificial neural networks to show that this object might be more similar to a Seyfert galaxy than other LINERs, from the X-ray point of view. The GNIRS spectrum (Figure A1.21) contains low ionization lines of [S III], [S II], $\mathrm{He} \mathrm{I}$, and $\left[\mathrm{Fe}_{\mathrm{II}}\right]$. [ $\left.\mathrm{C}_{\mathrm{I}}\right]$ is also present, along with possible weak, broad $\mathrm{Pa} \gamma$ and $\mathrm{Pa} \beta$. The strongest lines are from $\mathrm{He}$ I and [S III]. Moreover, He I shows some broadening. Mould et al. (2012) also presented a NIR spectrum of NGC 1052, showing prominent $\mathrm{Pa} \beta$ and $[\mathrm{Fe} \mathrm{II}]$ emission lines. In the $K$ band, the nuclear spectrum also includes $\mathrm{H}_{2}$ lines. The continuum shows $\mathrm{CaT}$ and $\mathrm{CO}$ absorption lines around 0.85 and $2.3 \mu \mathrm{m}$, as well as numerous stellar absorption features in the $H$ band. (Cid Fernandes et al. 2004) and find that the optical emission of NGC 1052 is mostly from an old stellar population, and Zhang et al. (2008) estimate a mean age of roughly $11 \mathrm{Gyr}$.

\section{NGC 3718 (L1.9)}

Morphologically, NGC 3718 is an SBa galaxy with a prominent dust lane crossing its central bulge. On kilopersec scales it is also known for its strongly warped atomic and molecular gas disk (Sparke et al. 2009). A type 1.9 LINER nucleus $\left(\mathrm{FWHM}_{\mathrm{H} \alpha}=2350 \mathrm{~km} \mathrm{~s}^{-1}\right.$; Ho et al. 1997a) lies behind this dust lane, with a compact jet extending to the northwest (Krips et al. 2007; Nagar et al. 2002). On large scales, NGC 3718 has a dwarf companion, NGC 3729 (Tully et al. 1996). Asymmetries found in $\mathrm{CO}(1-0)$ and $\mathrm{CO}(2-1)$ observations might be evidence for a tidal interaction with its companion at large scales and gas accretion onto the nucleus at small scales (Krips et al. 2005). The GNIRS spectrum (Figure A1.22) is continuum-dominated, with weak emission lines of $[\mathrm{S} \mathrm{III]}$, [Fe II], and He I. Stellar $\mathrm{CaT}$ and $\mathrm{CO}$ absorption bands are clearly detected near 0.85 and $2.3 \mu \mathrm{m}$, but little information about the stellar population of the nucleus of NGC 3718 is available in the literature.

\section{NGC 3998 L1.9}

Five galaxies (NGC 3990, NGC 3977, NGC 3972, NGC 3982, and UGC 6919) are seen within a projected distance of $250 \mathrm{kpc}$ of this S0 galaxy, all but one at redshifts consistent with sharing the same physical association (González-Martín et al. 2009b). A clear detection of a broad $\mathrm{H} \alpha$ line was reported by Heckman (1980) and Ho et al. (1997a). Longterm variability is widely reported at radio (Hummel et al. 1984; Filho et al. 2002), UV (Maoz et al. 2005), and X-ray (Younes et al. 2012; Hernández-García et al. 2013) frequencies. The nuclear stellar population of NGC 3998 appears very young, being dominated by stars of $10^{6}-10^{7} \mathrm{yr}$, although the blue continuum emission of the AGN in the high spatial resolution data used for the analysis renders this uncertain (González Delgado et al. 2004). The GNIRS spectrum of NGC 3998 (Figure A1.23) is full of emission lines, with the most prominent being $\mathrm{He}_{\mathrm{I}} 1.08 \mu \mathrm{m}$ (showing a clear broad component), $[\mathrm{S}$ III], and $[\mathrm{Fe} \mathrm{II}]$. [S II] and [NI] are also detected, and $\operatorname{Pa} \gamma$ and $\operatorname{Pa} \beta$ emission lines may also be present. At high angular resolution, the IR SED of this object is red and can be explained by emission from warm dust (Mason et al. 2013), but in these lower-resolution data the continuum shape is characteristic of stellar emission. The continuum is imprinted with the CaT and $\mathrm{CO}$ absorption bands, as well as numerous other weak, stellar features.

NGC 4203 (L1.9)

This AGN is hosted by a SAB0 galaxy seen nearly face-on, with a few dust lanes visible in the bulge. The LINER has prominent, double-peaked broad $\mathrm{H} \alpha$ lines (Shields et al. 2000) and fairly symmetric optical emission lines (Ho et al. 1997a). The AGN is also detected at various other wavelengths: as a point source in X-rays (Ho et al. 2001), as a variable UV source (Maoz et al. 2005), and as a point source with an inverted radio spectrum (Nagar et al. 2000). Mould et al. (2012) obtained an infrared spectrum with TripleSpec on the $5 \mathrm{~m}$ telescope at Palomar, and report that no emission lines were detected despite a $\mathrm{S} / \mathrm{N}$ near 100. Our spectrum (Figure A1.24) has a broad $\mathrm{He}_{\mathrm{I}}$ line and [S III] emission lines, probably made visible by the lesser dilution of the AGN spectrum in the 0.3 slit used for this work (cf. $1^{\prime \prime}$ used by Mould et al.). CaT and $\mathrm{CO}$ absorption bands are also visible, as well as numerous stellar absorption lines in the $H$ band. The nuclear stellar population of NGC 4203 has been examined by Sarzi et al. (2005). They find roughly equal $(\sim 45 \%)$ contributions from young $\left(\leqslant 10^{7.5} \mathrm{yr}\right)$ and old $\left(10^{10} \mathrm{yr}\right)$ components, but they note that the AGN featureless continuum in their high angular resolution data could be an alternative explanation for the apparent presence of large numbers of young stars.

NGC 4450 (L1.9)

Hosted in an SA(s)ab galaxy with medium inclination and soft spiral arms, this LINER has asymmetric narrow optical lines and a double-peaked, broad $\mathrm{H} \alpha$ line (Ho et al. 2000). VLA images reveal extended emission around the core (Anderson \& Ulvestad 2005), whose radio spectrum is flat or mildly inverted (Ho \& Ulvestad 2001). The GNIRS spectrum (Figure A1.25) has a few emission lines ([S III], He I, [Fe II $]$ ) and stellar absorption bands including $\mathrm{CaT}$ and $\mathrm{CO}$. Like NGC 4203, the nuclear stellar population of NGC 4450 has been examined by Sarzi et al. (2005). They find that young $\left(\leqslant 10^{7.5} \mathrm{yr}\right)$ and old $\left(\geqslant 10^{10} \mathrm{yr}\right)$ stars contribute roughly 40 and $60 \%$ of the optical light, respectively, but they note that the AGN featureless continuum in their high angular resolution data could be an alternative explanation for the apparent presence of large numbers of young stars.

NGC 4750 (L1.9)

The host of this AGN has an unusual morphology and is classified as a (R)SA(rs)ab galaxy. It has a very small, very bright nucleus with a nuclear bar. Additionally, images of the galaxy show a ring completely detached from the nucleus and probably consisting of two overlapping spiral arms (Erwin 2004). Ho et al. (1997b) detect faint wings in the $\mathrm{H} \alpha$ profile of this LINER and otherwise very symmetric narrow emission lines. Several emission lines (including [S III, [C I], $[\mathrm{Fe} I \mathrm{II}]$, and $\mathrm{H}_{2}$ ) are detected in the GNIRS spectrum (Figure A1.26), which rather closely resembles that of NGC 4450 except for the presence of the fairly strong $\mathrm{H}_{2}$ 
emission in the $K$ band. CaT, $\mathrm{CO}$, and many other stellar absorption features are also present.

NGC 5005 (L1.9)

NGC 5005 is a weakly barred spiral galaxy and part of a pair that also includes NGC 5033 (Helou et al. 1982). It is optically classified as a type 1.9 LINER (Ho et al. 1997a), and it has an intermediate-age stellar population contributing about $45 \%$ of the nuclear optical light (Cid Fernandes et al. 2004). The presence of a weak AGN is confirmed by the detection of a compact hard X-ray source (González-Martín et al. 2006; Dudik et al. 2009). $H$ - and $K$-band spectra of NGC 5005 were analyzed by Bendo \& Joseph (2004), who noted the presence of $\mathrm{H}_{2}$ and $[\mathrm{Fe}$ II] emission and the absence of detectable $\mathrm{Br} \gamma$. They interpreted the $\mathrm{H}_{2}$ and $[\mathrm{Fe}$ II] lines as indicative of shocks, which could be caused by supernovae, interaction with NGC 5033, or gas outflow from the AGN. All of the lines noted by Bendo \& Joseph (2004) are visible in the GNIRS spectrum of NGC 5005, along with additional $\mathrm{H}_{2}$ and $[\mathrm{Fe} \mathrm{II}]$ lines outside their spectral coverage. Emission from $[\mathrm{S} \mathrm{III}]$ and $\mathrm{HeI}$ is also detected, along with $\mathrm{CaT}$ and $\mathrm{CO}$ absorption, and numerous stellar absorption lines in the $H$ band.

\section{A.4 LINER 2}

\section{NGC 404 (L2)}

While the hard X-ray point source at the center of NGC 404 is faint enough that it could be an X-ray binary, the spectral shape of the $\mathrm{X}$-ray emission and the radio characteristics of this object suggest the presence of an accreting nuclear BH (Binder et al. 2011; Nyland et al. 2012). Modeling of the stellar kinematics implies that the mass of the $\mathrm{BH}$ is relatively low, $M_{\mathrm{BH}}<1 \times 10^{5} M_{\odot}$ (Seth et al. 2010). The nuclear star cluster of NGC 404 is fairly young, with stars $\sim 1$ Gyr old accounting for over half of the optical light in a $1^{\prime \prime}$ slit (Seth et al. 2010, see also Cid Fernandes et al. 2004). The GNIRS spectrum of NGC 404 (Figure A1.28) shows emission from [S III], [N I], He I, [Fe II], and $\mathrm{H}_{2}$, with stronger and more numerous lines than many of the other type 2 LINERs in this sample. This is probably a result of the galaxy's proximity $(D=2.4 \mathrm{Mpc})$, meaning that the nuclear emission is less diluted by stellar emission. Despite the presence of a 1 Gyr-old stellar cluster of roughly solar metallicity, deep molecular absorption features from TP-AGB stars, predicted by some stellar population models (Maraston 2005), are not visible in the IR spectrum.

NGC 474 (L2::)

NGC 474 (Arp 227) is a lenticular galaxy surrounded by arcs or shells of stars (Turnbull et al. 1999) that were possibly formed in an interaction with neighboring NGC 470, or in a past merger event. The central regions of the galaxy are old $(\sim 9 \mathrm{Gyr})$, while the age of the stellar population decreases toward the galaxy's outskirts (Kuntschner et al. 2010). The classification of NGC 474 as a type 2 LINER is highly uncertain, and its optical emission lines are weak (Ho et al. 1997a). If an AGN is present in NGC 474, it may be deeply obscured (González-Martín et al. 2009a). No emission lines are detected in the IR spectrum (Figure A1.29), and the good S/N and excellent inter-band telluric line removal in the data clearly reveal the nuclear stellar population. Molecular absorption features at $\sim 0.93 \mu \mathrm{m}$ (probably a blend of $\mathrm{TiO}$, $\mathrm{ZrO}$, and $\mathrm{CN})$ and $1.1 \mu \mathrm{m}(\mathrm{CN})$ are relatively strong in this object.
NGC 1961 (L2)

Studies of the CO emission in NGC 1961 (UGC 3334) suggest that the galaxy once collided with a companion, and that its disk has since become strongly warped (Combes et al. 2009). Consideration of the radio and infrared properties of this galaxy led to its classification as a starburst by Condon et al. (1991), and it has been host to several recent supernovae. Dust lanes are visible in the optical image of this inclined, SABc galaxy (Sandage \& Bedke 1994), and nondetection of a nuclear UV source also suggests that it may be fairly dusty (Barth et al. 1998). This is consistent with the downturn in flux at short wavelengths in the GNIRS spectrum (Figure A1.30). Lines from species such as [S III], [Fe II], and He I are present, although fairly weak, and numerous, prominent emission lines of $\mathrm{H}_{2}$ are present in the $K$ band.

NGC 2768 (L2)

This polar ring elliptical galaxy is detected in $\mathrm{CO}$, and may have acquired material during a tidal accretion event (Crocker et al. 2008). Sil'chenko \& Afanasiev (2006) derive a luminosity-weighted mean age of $11 \mathrm{Gyr}$ for the galaxy's nucleus, although Crocker et al. (2008) and Kuntschner et al. (2010) find evidence of a star-forming episode occurring a few Gyr ago, Zhang et al. (2008) find a mean age of $~ 6 \mathrm{Gyr}$, and Serra et al. (2008) estimate a mean age of roughly 4 Gyr. In any case, there is little evidence of ongoing star formation. NGC 2768 is host to a compact radio source and variable X-ray nucleus (Nagar et al. 2005; Filho et al. 2006; Komossa et al. 1999), and there are weak emission lines in its optical spectrum (Ho et al. 1995). The GNIRS spectrum (Figure A1.31) is practically devoid of emission lines, with a couple of $\mathrm{H}_{2}$ perhaps weakly detected. The continuum shape and absorption features are broadly similar to those of several of the other type 2 LINERs in this sample.

NGC 2832 (L2::)

NGC 2832 is an elliptical galaxy and the brightest galaxy of the Abell 779 cluster. It may be interacting with a satellite galaxy, NGC 2831 (Lauer 1988). The object has some weak optical line emission, leading to an uncertain LINER 2 classification (Ho et al. 1997a), but evidence of AGN activity has not been forthcoming (for instance, the nucleus has not been detected in VLBA and VLA observations; Nagar et al. 2005; Liuzzo et al. 2010). The central stellar population of NGC 2832 is found to be old, with derived ages of 7.5-13 Gyr (Proctor \& Sansom 2002; Sánchez-Blázquez et al. 2006; Zhang et al. 2008; Loubser et al. 2009). No emission lines are detected in the IR spectrum (Figure A1.32), which otherwise broadly resembles those of the other type 2 LINERs presented here.

\section{NGC 3169 (L2)}

The Sa galaxy NGC 3169 is part of a gas-rich interacting group that also includes NGC 3166 and NGC 3156. NGC 3169 hosts a low-luminosity AGN, revealed by the presence of a hard X-ray point source (Satyapal et al. 2005) and compact, high brightness temperature radio source (Falcke et al. 2000; Nagar et al. 2005). According to Cid Fernandes et al. (2004), the optical emission is predominantly from old stars, although with a minor contribution $(21 \%)$ from an intermediate-age $\left(10^{8}-10^{9} \mathrm{yr}\right)$ population (see also Sil'chenko \& Afanasiev 2006). There is weak $\mathrm{H}_{2}$ emission in the GNIRS spectrum of NGC 3169 (Figure A1.33), and possibly [Fe II] $1.257 \mu \mathrm{m}$ as well. The continuum shows absorption bands of both atomic and 
molecular origin, qualitatively similar to those observed in many of the other type 2 LINERs in this sample. The flattening at the short wavelength end is probably due to extinction, as the nucleus of this object contains chaotic, dusty structures (González Delgado et al. 2008).

NGC 3190 (L2)

Very little-studied to date, NGC 3190 is a highly inclined spiral galaxy and part of the HGC44 group. In common with several other LINER $2 \mathrm{~s}$ in this sample, the IR spectrum of this object (Figure A1.34) shows just a handful of very weak emission lines, in this case from $\left[\mathrm{C}_{\mathrm{I}}\right],\left[\mathrm{N}_{\mathrm{I}}\right], \mathrm{Fe} \mathrm{II}$, and $\mathrm{H}_{2}$.

NGC 3607 (L2)

Along with NGC 3605 and NGC 3608, NGC 3607 forms part of the G49 (Leo II) group (de Vaucouleurs 1975). X-ray observations show no clear evidence for the presence of an AGN (Terashima et al. 2002; Flohic et al. 2006; GonzálezMartín et al. 2009b). Estimates of the age of the central stellar population vary. Rickes et al. (2009) find that most of the optical emission arises in components of $\geqslant 5 \mathrm{Gyr}$, and Proctor \& Sansom (2002) find a mean age of 5.6 Gyr. Annibali et al. (2007) and Terlevich \& Forbes (2002) find younger average ages of 3 Gyr, but Sil'chenko \& Afanasiev (2006) estimate a luminosity-weighted age of $12 \mathrm{Gyr}$. The IR spectrum of NGC 3607 (Figure A1.35) is qualitatively similar to those of many of the other type 2 LINERs in this sample, and only one or two weak emission lines (from $\left[\mathrm{S}\right.$ III] and perhaps $\mathrm{H}_{2}$ ) are detected.

NGC 4346 (L2::)

NGC 4346 appears to be an unremarkable S0 galaxy, with few, weak optical emission lines, and a tentative LINER 2 classification. To date, it is undetected in X-rays (Halderson et al. 2001) and in radio surveys aimed at searching for nuclear activity (Nagar et al. 2005). The good telluric line removal, high $\mathrm{S} / \mathrm{N}$, and lack of detectable emission lines in the GNIRS spectrum (Figure A1.36) make this object useful for detailed examination of stellar features in the IR.

NGC 4548 (L2)

NGC 4548 is an "anemic" spiral galaxy whose HI gas deficiency may have been caused by ram pressure stripping as it passed close to the center of the Virgo cluster (Vollmer et al. 1999). The nuclear stellar population is old, $\sim 10 \mathrm{Gyr}$ (Sarzi et al. 2005), and X-ray emission from a low-luminosity and somewhat obscured AGN is detected (Terashima \& Wilson 2003; Eracleous et al. 2010b). The GNIRS spectrum (Figure A1.37) contains only a few, very weak emission lines, and is generally rather similar to most of the other LINER 2 galaxies in this sample.

\section{NGC 4594 (L2)}

NGC 4594 (M104; the Sombrero galaxy) has been extensively studied from radio to gamma-ray wavelengths. The edge-on host contains a weak AGN accompanied by parsec-scale jets (Hada et al. 2013; Mezcua \& Prieto 2014), and at sub-arcsecond angular resolution the IR part of the SED appears to be dominated by synchrotron emission (Mason et al. 2012). The lower spatial resolution GNIRS spectrum (Figure A1.38) shows no clear sign of this, being similar in shape to the other type 2 LINERs in the sample. Only a few, weak emission lines of $[\mathrm{S} \mathrm{III]}$ and $[\mathrm{Fe}$ II $]$ are tentatively detected. The central stellar population of NGC 4594 has been studied by Sánchez-Blázquez et al. (2006), who find ages ranging from 6 to 12 Gyr, depending on the optical line index used.
NGC 4736 (L2)

NGC 4736 is a face-on, ringed galaxy also known as M94. The galaxy hosts a multitude of nuclear X-ray sources, likely the consequence of an intense period of star formation (Eracleous et al. 2002). However, the presence of a variable UV source and compact radio core suggest that it also hosts a low-luminosity AGN (Maoz et al. 2005; Nagar et al. 2005). Cid Fernandes et al. (2004) find that just under half of the nuclear $4020 \AA$ emission of NGC 4736 is from a $10^{8}-10^{9}$ yr-old stellar population, and Taniguchi et al. (1996) classify the galaxy as a post-starburst system on the basis of its optical Balmer absorption lines. The GNIRS spectrum (Figure A1.39) is of somewhat lower $\mathrm{S} / \mathrm{N}$ than most of the other spectra in this paper. A couple of weak emission lines may be present in the data, and the continuum shape is broadly similar to that of many of the other types 2 LINERs discussed here.

NGC 5371 (L2)

NGC 5371, a weakly barred spiral galaxy, hosts a lowluminosity hard X-ray point source (Cisternas et al. 2013) that has not been detected in MERLIN or VLA radio observations (Nagar et al. 2005; Filho et al. 2006). No emission lines are detected in the IR spectrum (Figure A1.40), in agreement with the $H$ - and $K$-band data of Bendo \& Joseph (2004).

NGC 5850 (L2)

NGC 5850 is a double-barred spiral galaxy that may have survived an encounter with a nearby massive elliptical galaxy, NGC 5846 (Higdon et al. 1998). The optical line emission from NGC 5850 is spatially extended, and sources of ionization may include post-AGB stars and low-level star formation (Bremer et al. 2013). de Lorenzo-Cáceres et al. (2013) observe spiral structures and twisted velocity fields that suggest the presence of gas streaming toward the nucleus, and find that the central stellar population of NGC 5850 has a luminosityweighted age in the region of $5 \mathrm{Gyr}$. In contrast, the IR spectrum of NGC 5850 (Figure A1.41) is almost devoid of lines, with only $\mathrm{H}_{2}$ emission being weakly detected.

NGC 6500 (L2)

NGC 6500 contains a compact, flat-spectrum radio core (Falcke et al. 2000; Filho et al. 2002), variable UV nucleus (Maoz et al. 2005), and nuclear hard X-ray source (Terashima \& Wilson 2003; Dudik et al. 2005) that indicate the presence of an AGN in this spiral galaxy. The optical LINER emission is extended, with kinematics suggestive of an outflowing wind (Gonzalez Delgado \& Perez 1996). Most of the nuclear optical light in NGC 6500 comes from an old stellar population, although about $25 \%$ appears to arise in young $\left(<10^{7} \mathrm{yr}\right)$ stars (Cid Fernandes et al. 2004). Unlike most of the LINER 2 nuclei in this sample, the GNIRS spectrum of NGC 6500 (Figure A1.42) contains numerous, fairly prominent emission lines, from species including $[\mathrm{S} \mathrm{III}], \mathrm{He} \mathrm{I}$, and [Fe II].

NGC 7217 (L2)

This galaxy contains a number of star-forming rings (e.g., Buta et al. 1995; Sarzi et al. 2007), which lie outside the slit used for these observations, and the gas within the innermost ring is currently outflowing (Combes et al. 2004). The nuclear stellar population is predominantly old, with just a few per cent of the optical light being emitted by intermediate-age $\left(10^{8}-10^{9}\right.$ yr) stars (Cid Fernandes et al. 2004). The GNIRS spectrum (Figure A1.43) contains several emission lines, of which those of $[\mathrm{Fe}$ II $]$ and $\mathrm{H}_{2}$ are strongest. The overall continuum shape qualitatively resembles that of most of the other type 2 LINERs in this work. 


\section{A.5 TOs, Inactive Galaxies}

NGC 410 (T2:)

A relatively little-studied elliptical galaxy, NGC 410 is the central galaxy of the Pisces cluster (Hudson et al. 2001). The S/ $\mathrm{N}$ in the GNIRS spectrum is relatively low, but fairly strong stellar absorption bands at 1.1 and $0.93 \mu \mathrm{m}$ are clearly visible. No identifiable emission lines are reliably detected in the NIR spectrum, although weak $\mathrm{H} \beta, \quad\left[\mathrm{N}_{\mathrm{II}}\right] \lambda 6583$, and $\left[\mathrm{S}_{\mathrm{II}}\right]$ $\lambda \lambda 6716,6731$ are present in the optical spectrum of Ho et al. (1997a). Stellar population synthesis indicates that the optical light of NGC 410 comes almost entirely from old stars $\left(>10^{9}\right.$ yr; Cid Fernandes et al. 2004; González Delgado et al. 2004).

NGC 660 (T2/H:)

NGC 660 is a highly inclined, barred spiral galaxy with a polar ring (Whitmore et al. 1990; Combes et al. 1992) and a rather high IR luminosity $\left(\log L_{\mathrm{IR}}=10.5 L_{\odot}\right.$; Sanders et al. 2003) that indicates ongoing star formation. Based on optical spectroscopy, Cid Fernandes et al. (2004) find a significant intermediate-age population in the nuclear regions of this galaxy, with over half of the $4020 \AA$ flux coming from stars of $10^{8}-10^{9} \mathrm{yr}$. The detection of the $[\mathrm{Ne} \mathrm{v}] \lambda 14.32$ line confirms that NGC 660 hosts an AGN (Goulding \& Alexander 2009), with a deep silicate absorption feature that shows it to be highly obscured (Roche et al. 1991). The GNIRS spectrum of NGC 660 (Figure A1.45) is unique among the 50 sources observed. It displays a prominent excess of NIR emission, particularly in the $K$ band, where the continuum flux increases steeply with increasing wavelength. This spectral shape is very similar to that observed in NGC 1068 and Mrk 1239 (Rodríguez-Ardila et al. 2006; Martins et al. 2010), so we attribute it to emission from hot dust. Strong hydrogen recombination lines are observed, along with a series of fairly prominent $[\mathrm{Fe}$ II $]$ and $[\mathrm{S}$ III $]$ lines.

NGC 4569 (T2)

NGC 4569 is a large, anaemic (gas-poor) spiral galaxy in the Virgo cluster (van den Bergh 1976), and observations of its $\mathrm{H} \mathrm{I}$ content suggest that a ram pressure stripping event occurred around $300 \mathrm{Myr}$ ago (Vollmer et al. 2004). The nuclear UV spectrum of NGC 4569 indicates the presence of a young (5-6 Myr) starburst (Maoz et al. 1998; Gabel \& Bruhweiler 2002), while the optical spectrum shows strong, narrow Balmer lines that reveal a population of A-type supergiants (Keel 1996) and a generally young-intermediate age population (Cid Fernandes et al. 2004). Recent and/or ongoing star formation activity is also suggested by the detection of polycyclic aromatic hydrocarbon (PAH) emission in the MIR (Dale et al. 2006). Any spectral signatures of a post-starburst population in NGC 4569 must fairly subtle, as the NIR absorption line spectrum of this nucleus (Figure A1.46) looks broadly similar to those of many other objects in this study. A handful of common emission lines are observed in the spectrum, from $\left[\mathrm{S}_{\mathrm{III}}\right], \mathrm{He} \mathrm{I}$, and $[\mathrm{Fe} \mathrm{II}]$.

NGC 7331 (T2)

NGC 7331 (Figure A1.46) is a highly inclined spiral galaxy in the vicinity of Stephan's quintet, although it is not associated (Gutiérrez et al. 2002). The galaxy contains a ring of star formation $(r \sim 6 \mathrm{kpc})$ visible at infrared and longer wavelengths (e.g., Telesco et al. 1982), from which material appears to be flowing inwards (Battaner et al. 2003). MIR spectra covering the nucleus and inner ring show emission in several $\mathrm{H}_{2}$ and fine structure lines, and the detection of [O IV $] 25.9 \mu \mathrm{m}$ emission at the nucleus implies the presence of either an AGN,
Wolf-Rayet stars, or shocks (Smith et al. 2004). PAH emission is also detected at the nucleus, as well as in the ring.

In contrast, the nuclear optical spectrum of NGC 7331 shows only weak emission from $\mathrm{H} \beta,[\mathrm{O}$ III $] \lambda 5007$, [N $\mathrm{NI}] \lambda 6583$, and [S II $] \lambda \lambda$ 6716,6731. Its Balmer absorption lines led Ohyama \& Taniguchi (1996) to classify NGC 7331 as a post-starburst galaxy, with $\sim 40 \%$ of the optical light being produced by stars with ages $\sim 5 \times 10^{9}$ yr. Cid Fernandes et al. (2004) come to a different conclusion, finding only a $5 \%$ contribution from intermediate-age stars. Spectral features from any post-starburst population are not obvious in the NIR spectrum of this object. The GNIRS spectrum (Figure A1.47) is almost devoid of emission lines; the energetic processes leading to the $\mathrm{PAH}$ and [O IV] lines in the MIR spectrum leave little trace in the NIR data.

\section{NGC 205 (Inactive)}

NGC 205 is a dwarf elliptical galaxy and a close satellite of M31. Given its proximity, it has been extensively studied at many wavelengths. No evidence of AGN activity has been detected to date (Ho et al. 1997a; Colbert \& Mushotzky 1999), and any $\mathrm{BH}$ at the nucleus must have a mass of no more than a few $\times 10^{4} M_{\odot} \quad($ Valluri et al. 2005). No emission lines are visible in the optical spectrum of NGC 205 presented by Ho et al. (1995), but deep HST photometry reveals a population of young blue stars at its center (Monaco et al. 2009), and the optical spectrum displays a blue continuum and strong Balmer absorption lines indicative of A stars (Bica et al. 1990; González Delgado et al. 1999; Cid Fernandes et al. 2004). The GNIRS spectrum (Figure A1.48) is similarly free from detectable emission lines. Qualitatively it is rather similar to those of the LINERs and TOs in this sample, but many of the absorption lines are shallower and the broad H-band "bump" (Section 4.1) appears somewhat weaker than in many other objects. The stellar population responsible for the optical Balmer lines leaves no gross signatures in the IR spectrum of NGC 205.

\section{A.6 Non-Palomar Galaxies}

\section{H 1934-063 (NLS1)}

1H 1934-063 is the only narrow-line Seyfert 1 AGN in the sample. It has been studied in the optical and NIR because of its prominent spectrum, rich in emission lines both from the NLR and BLR. Rodríguez-Ardila et al. (2002) were the first to publish a NIR spectrum of this source, dominated by broad lines from Fe II, O I, He I, and $\mathrm{H}_{\mathrm{I}}$. Narrow, bright forbidden lines from $[\mathrm{S}$ III] and prominent coronal lines of [S VIII], [S IX], [Si vi], [Si x], and [Ca VIII] are also easily seen in its NIR spectrum. The GNIRS spectrum (Figure A1.50) shows all these lines in addition to [Si VII] at $2.48 \mu \mathrm{m}$, which was not previously reported. The main difference between the present and previous NIR spectroscopy is that the continuum in the GNIRS spectrum is flatter. This is probably because the 0.3 slit used for these observations is significantly smaller than that of previous observations (0!.8) and therefore includes less emission from the host galaxy. Otherwise, the spectroscopy presented here matches very well previous observations covering a similar wavelength interval.

\section{NGC 7469 (S1)}

This Seyfert 1 galaxy has been extensively studied in the literature. Its most prominent characteristic is a circumnuclear ring (1".5-2".5) of powerful starburst activity observed at radio, optical, NIR, and MIR wavelengths (Davies 


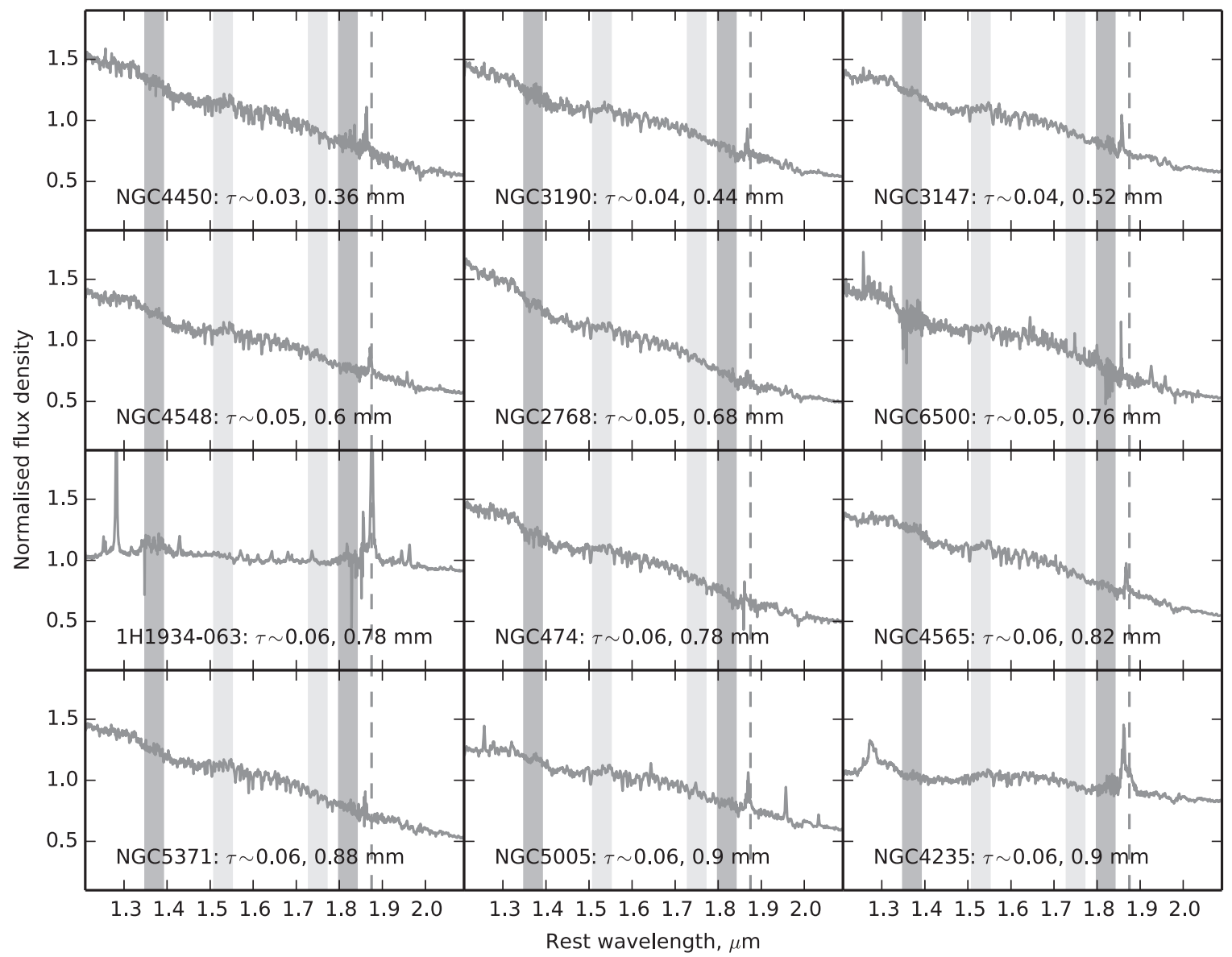

Figure B1. Galaxy spectra arranged in order of increasing $\tau_{225 \mathrm{GHz}}$. The shaded areas indicate regions used to measure the S/N plotted in Figure B2. The dashed vertical line marks the wavelength of the Pa $\alpha$ emission line, which is not present in all the spectra but could be quite easily confused with a neighboring residual that appears in almost every data set.

(An extended version of this figure is available.)

et al. 2004, and references therein), with a luminosity that amounts to two-thirds of the bolometric luminosity of the entire galaxy. NIR spectroscopy of this source has been published by several authors including Genzel et al. (1995), Thompson (1996), Sosa-Brito et al. (2001), Riffel et al. (2006), and Davies et al. (2007). The GNIRS emission line spectrum (Figure A1.49) agrees well with that presented by Riffel et al. (2006). The main difference is found in the continuum, with the GNIRS spectrum displaying an excess of continuum over the underlying power-law at the red end of the spectrum, peaking at $\sim 2.2 \mu \mathrm{m}$. This feature can be interpreted as emission from dust heated to its sublimation temperature $(\sim 1800 \mathrm{~K})$ by the AGN. Although this excess was also detected by Riffel et al. (2006), it is more conspicuous in the GNIRS spectrum, probably due to the smaller slit width which isolates the NIR emission from the host galaxy and circumnuclear ring of star formation. He I $1.083 \mu \mathrm{m}$ and $\left[\mathrm{S}_{\mathrm{III}}\right]$ 0.907, $0.953 \mu \mathrm{m}$ are the most conspicuous lines observed, and high-ionization lines of [S VIII], [S Ix], [Si vI $],[\mathrm{Si}$ VII $]$, and $[\mathrm{Six}]$ are also identified. Stellar absorption features are detected mostly in the $H$ and $K$ bands, and the $2.3 \mu \mathrm{m} \mathrm{CO}$ bandheads are prominent. The CaT at $0.85 \mu \mathrm{m}$ is also present but is affected by the superimposed, broad O I $0.845 \mu \mathrm{m}$ emission line.

\section{APPENDIX B \\ ATMOSPHERIC WATER VAPOR AND INTER-BAND S/N}

The regions between the traditional NIR bands suffer from poor atmospheric transmission and strong absorption from telluric molecules (Figure A2). Access to these regions can be desirable, however, for studies of features such as $\mathrm{H}_{2} \mathrm{O}$ and $\mathrm{C}_{2}$ bands in evolved stellar populations, or emission lines in redshifted galaxies. The main atmospheric absorber in these inter-band regions is $\mathrm{H}_{2} \mathrm{O}$, while $\mathrm{CO}_{2}$ also contributes around 1.4, 1.6, and (especially) $2.0 \mu \mathrm{m}$ (Glass 1999; Seifahrt et al. 2010). Telluric water vapor is monitored at several observatory sites, giving observers the opportunity to adapt their observing strategy to the prevailing conditions. To the best of our knowledge, though, the empirical relationship between atmospheric water vapor and the $\mathrm{S} / \mathrm{N}$ in the inter-band regions of the final spectrum, after dividing by a standard star to remove the telluric lines, has not yet been presented in an easily accessible form. In the GNIRS data set we have many pairs of galaxies and standard stars observed under a wide range of observing conditions. We therefore use the galaxy spectra to explore the effect of telluric water vapor on data quality in the regions of the spectrum around 1.35 and $1.85 \mu \mathrm{m}$.

First, Figures B1-B1.4 present the reduced spectra arranged in order of increasing $225 \mathrm{GHz}$ zenith optical depth $\left(\tau_{225 \mathrm{GHz}}\right)$, 


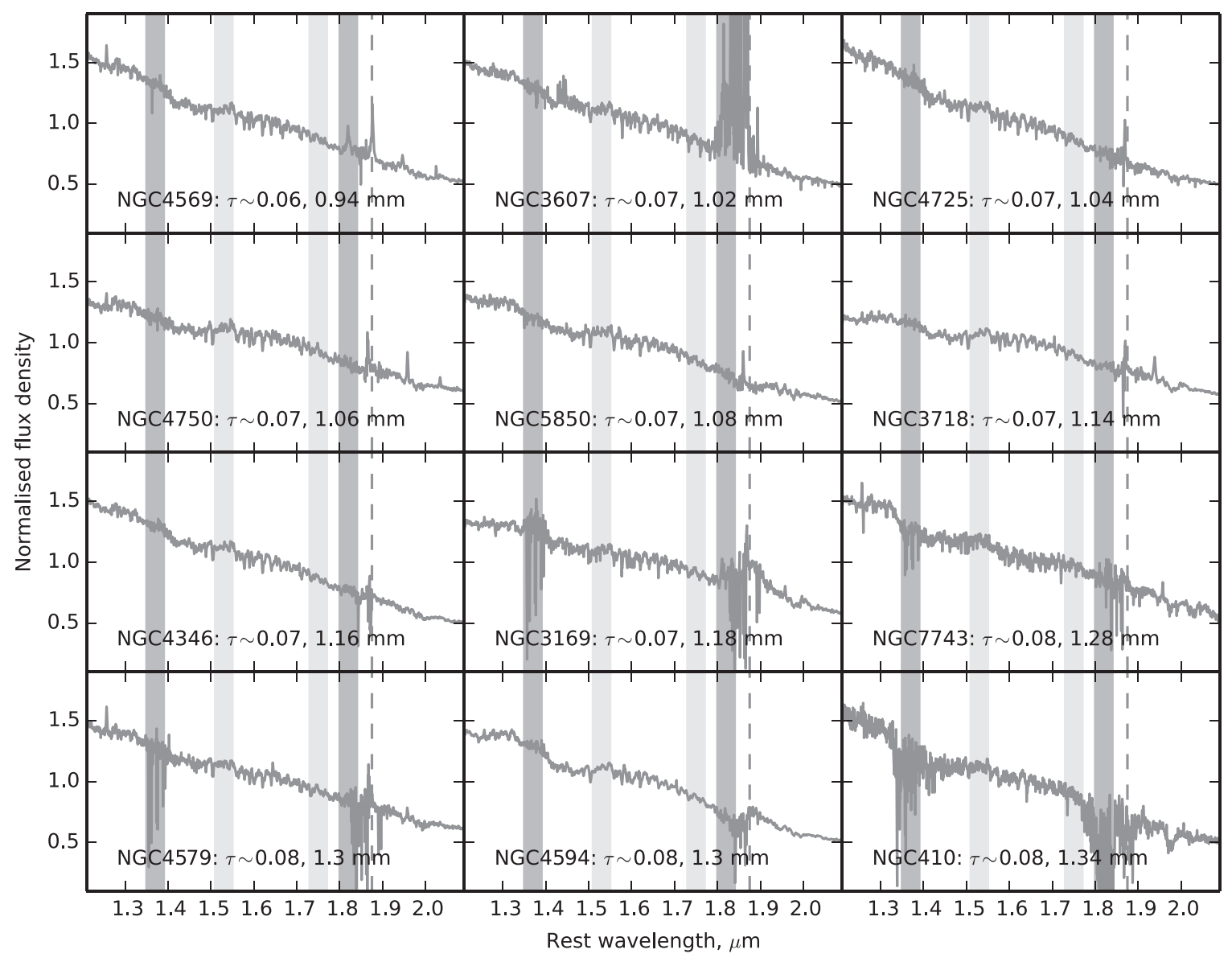

Figure B1. (Continued.)

or atmospheric water vapor column (WV), at the start of the observation. ${ }^{25}$ The $225 \mathrm{GHz}$ optical depth is measured every 10 minutes by a tipping radiometer at the Caltech Submillimeter Observatory (Radford 2011), and is related to the water vapor column above Mauna Kea as WV $=20\left(\tau_{225} \mathrm{GHz}-0.016\right) \mathrm{mm}$ (Davis et al. 1997). ${ }^{26}$ The atmospheric water vapor column varies with elevation and azimuth angle (Naylor et al. 2002), so $\tau_{225 \mathrm{GHz}}$ is not necessarily an accurate indication of the water vapor column affecting the GNIRS observations. Nonetheless, the trend to lower $\mathrm{S} / \mathrm{N}$ in the inter-band regions with increasing $\tau_{225 \mathrm{GHz}}$ is clear. NGC 2768 , for instance, observed at $\tau_{225 \mathrm{GHz}} \sim 0.05$ ( $\mathrm{WV} \sim 0.7 \mathrm{~mm}$; Figure B1), displays a very clean spectrum, whereas the inter-band regions of NGC 1358 $\left(\tau_{225 \mathrm{GHz}} \sim 0.27 ; \mathrm{WV} \sim 5 \mathrm{~mm}\right.$; Figure B1.4) are unusably noisy. Roughly speaking, the inter-band regions tend to be fairly free of residuals from dividing by the standard star up to $\tau_{225 \mathrm{GHz}} \sim 0.07 \quad(\mathrm{WV} \sim 1.1 \mathrm{~mm})$. Regardless of the water vapor, however, almost all spectra display at least a small residual just shortwards of the $\mathrm{Pa} \alpha$ wavelength of $1.875 \mu \mathrm{m}$, where the atmosphere is virtually opaque even in the best conditions.

In Figure $\mathrm{B} 2$ we show the $\mathrm{S} / \mathrm{N}$ achieved in the galaxy spectra in two areas of strong $\mathrm{H}_{2} \mathrm{O}$ absorption. On the whole,

\footnotetext{
${ }^{25}$ Excluding NGC 2639, NGC 2655, NGC 2832, and NGC 3079, for which no $\tau$ data are available, and NGC 315 and NGC 4395, whose poor sky subtraction makes them unsuitable for this analysis.

${ }^{26}$ See Thomas-Osip et al. (2007) for other observing sites.
}

the $\mathrm{S} / \mathrm{N}$ in these inter-band regions increases markedly as the WV decreases. At 1.35-1.39 $\mu \mathrm{m}$ (between the $J$ and $H$ bands), $\mathrm{S} / \mathrm{N} \sim 30-70$ is often achieved below $\mathrm{WV} \sim 1.3 \mathrm{~mm}$. Improvement is also seen at $1.81-1.85 \mu \mathrm{m}$ (between $H$ and $K$ ), with $\mathrm{S} / \mathrm{N} \sim 20$ quite frequently being reached in dry conditions. There is considerable scatter in the plots, though, particularly at $1.81-1.85 \mu \mathrm{m}$. The point at $1.2 \mathrm{~mm}, \mathrm{~S} / \mathrm{N}=5.4$ in the righthand plot is NGC 3169 . The airmass match between this galaxy and its standard star was unusually poor, which may account for its unusually low $\mathrm{S} / \mathrm{N}$ given the water vapor. There is no obvious reason for the other points in the lower left corner of the plot, which may instead be related to random spatial and temporal variations in the atmosphere.

While the trend in $\mathrm{S} / \mathrm{N}$ versus $\tau$ is clear, the absolute values of the $\mathrm{S} / \mathrm{N}$ obtained in very dry conditions are less straightforward to interpret. They may either indicate the limit on the achievable $\mathrm{S} / \mathrm{N}$ imposed by systematic errors from the division by the standard star, or they may reflect the $\mathrm{S} / \mathrm{N}$ that could be achieved for each object in the exposure time used for the observations, or other effects. For comparison, we also show the $\mathrm{S} / \mathrm{N}$ in neighboring, relatively "clean" spectral regions. As expected, the $\mathrm{S} / \mathrm{N}$ in those regions is not related to the water vapor column. In this particular data set, the $\mathrm{S} / \mathrm{N}$ achieved at $1.35-1.39 \mu \mathrm{m}$ in dry conditions is comparable to that obtained in a relatively absorption-free region of the $H$ band at $1.51-1.55 \mu \mathrm{m}$. At $1.81-1.85 \mu \mathrm{m}$, the $\mathrm{S} / \mathrm{N}$ is systematically lower than that reached at $1.73-1.77 \mu \mathrm{m}$, regardless of the conditions. 


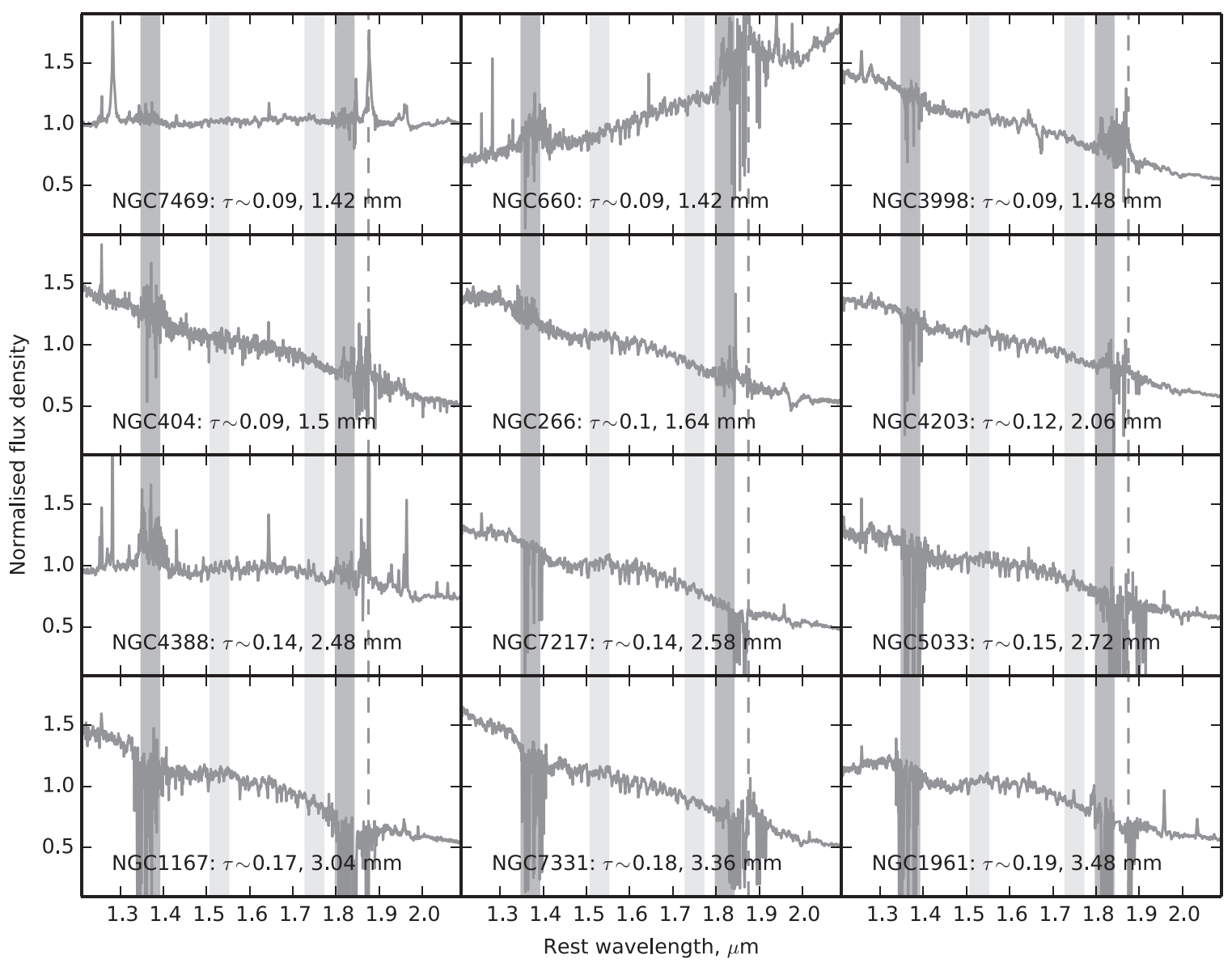

Figure B1. (Continued.)

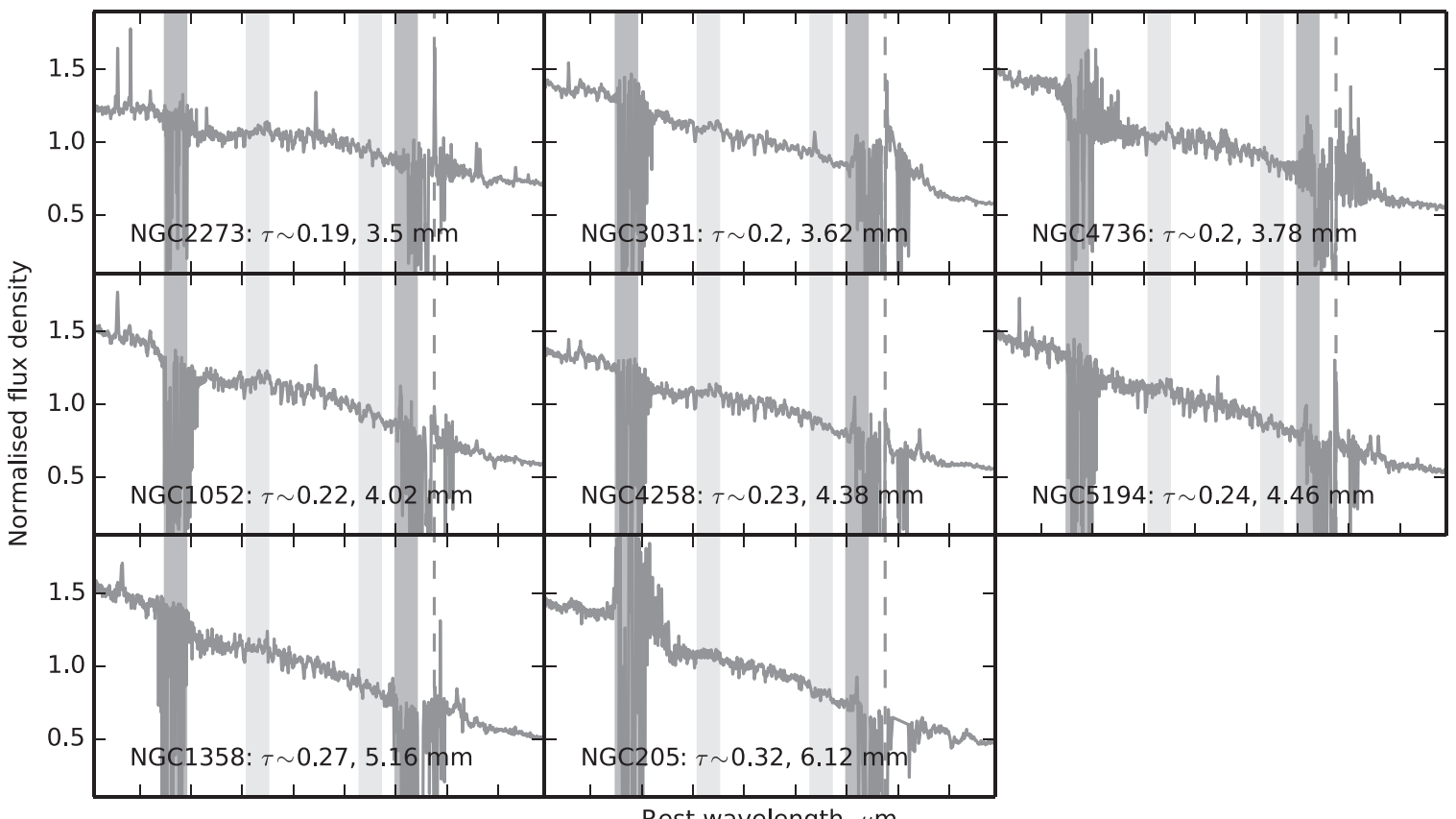

Figure B1. (Continued.) 

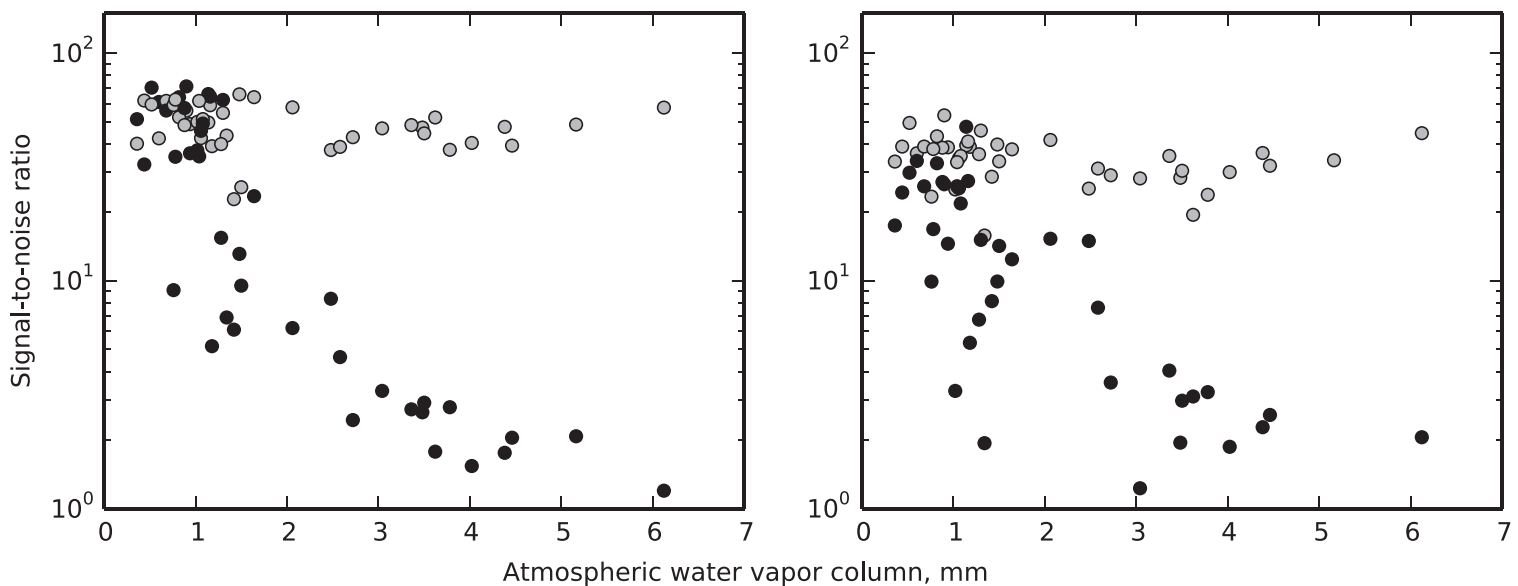

Figure B2. S/N vs. telluric water column, in the spectral regions shown in Figures A2-B1.4. Left: 1.35-1.39 $\mu \mathrm{m}$ (black) and 1.51-1.55 $\mu \mathrm{m}$ (gray). Right: $1.80-1.84 \mu \mathrm{m}$ (black) and $1.73-1.77 \mu \mathrm{m}$ (gray).

The fact that we obtain similar $\mathrm{S} / \mathrm{N}$ at $1.35-1.39$ and $1.51-1.55 \mu \mathrm{m}$ is likely an artifact of our simple definition of the $\mathrm{S} / \mathrm{N}$ as the mean/rms of the points in that region. All else being equal, the $\mathrm{S} / \mathrm{N}$ at $\sim 1.35 \mu \mathrm{m}$ will be lower than that at $\sim 1.55 \mu \mathrm{m}$, simply because the strong atmospheric absorption means that the telescope receives fewer photons from the astronomical object at those wavelengths. The fact that the $\mathrm{S} / \mathrm{N}$ is similar in regions of high and low transmission implies that systematic effects are limiting the measured $\mathrm{S} / \mathrm{N}$ in the regions of high transmission. In this case, this likely results from the fact that the galaxy spectra contain real spectral structure that contributes to the measurements of the rms in the spectrum. When similar $\mathrm{S} / \mathrm{N}$ is reached in regions of low transmission, we are probably also measuring similar spectral structure.

Observations of pairs of featureless standard stars, which were not obtained as part of this observing program, would enable a more in-depth exploration of the limiting $\mathrm{S} / \mathrm{N}$ that can be achieved in dry conditions. Nonetheless, we can draw some general conclusions. On one hand, it is possible to achieve $\mathrm{S} / \mathrm{N}$ of several tens even in regions of very poor atmospheric transmission when the atmospheric water vapor column $\lesssim 1 \mathrm{~mm}$. On the other hand, observing when the local $\tau$ meter indicates low water vapor is not a foolproof means of attaining high S/N: the relation between $\mathrm{S} / \mathrm{N}$ and $\tau$ in Figure B2 contains considerable scatter. Also, it is important to realize that the atmospheric transmission is zero under many of the narrow lines that are blended together in Figure A2 (see e.g., the highresolution Mauna Kea transmission spectrum shown by Cotton et al. 2014). This means that if a narrow spectral feature lies at one of these wavelengths, it will not be detected in the final spectrum even if the telluric line cancellation is very good.

A number of groups have been investigating correcting for telluric absorption using atmospheric models (e.g., Bailey et al. 2007; Seifahrt et al. 2010; Cotton et al. 2014; Gullikson et al. 2014). Until these methods are in widespread use, we hope that the analysis presented here will aid the planning of observations at the edges of the NIR atmospheric windows.

\section{REFERENCES}

Akylas, A., \& Georgantopoulos, I. 2009, A\&A, 500, 999

Alonso-Herrero, A., Rieke, M. J., Rieke, G. H., \& Ruiz, M. 1997, ApJ, 482,747

Alonso-Herrero, A., Rieke, M. J., Rieke, G. H., \& Shields, J. C. 2000, ApJ, 530,688
Anderson, J. M., \& Ulvestad, J. S. 2005, ApJ, 627, 674

Annibali, F., Bressan, A., Rampazzo, R., Zeilinger, W. W., \& Danese, L. 2007, A\&A, 463, 455

Asari, N. V., Cid Fernandes, R., Stasińska, G., et al. 2007, MNRAS, 381,263

Asmus, D., Gandhi, P., Smette, A., Hönig, S. F., \& Duschl, W. J. 2011, A\&A, 536, A36

Bailey, J., Simpson, A., \& Crisp, D. 2007, PASP, 119, 228

Balmaverde, B., \& Capetti, A. 2014, A\&A, 563, A119

Barth, A. J., Filippenko, A. V., \& Moran, E. C. 1999, ApJ, 525, 673

Barth, A. J., Ho, L. C., Filippenko, A. V., \& Sargent, W. L. W. 1998, ApJ, 496, 133

Battaner, E., Mediavilla, E., Guijarro, A., Arribas, S., \& Florido, E. 2003, A\&A, 401, 67

Bender, R., Burstein, D., \& Faber, S. M. 1993, ApJ, 411, 153

Bendo, G. J., \& Joseph, R. D. 2004, AJ, 127, 3338

Bianchi, S., Corral, A., Panessa, F., et al. 2008, MNRAS, 385, 195

Bica, E., Alloin, D., \& Schmidt, A. A. 1990, A\&A, 228, 23

Binder, B., Williams, B. F., Eracleous, M., et al. 2011, ApJ, 737, 77

Binette, L., Magris, C. G., Stasińska, G., \& Bruzual, A. G. 1994, A\&A, 292, 13

Blanco, P. R., Ward, M. J., \& Wright, G. S. 1990, MNRAS, 242, 4P

Boisson, C., Joly, M., Moultaka, J., Pelat, D., \& Serote Roos, M. 2000, A\&A, 357,850

Bouchard, A., Prugniel, P., Koleva, M., \& Sharina, M. 2010, A\&A, 513, A54

Braatz, J. A., Wilson, A. S., \& Henkel, C. 1994, ApJL, 437, L99

Bremer, M., Scharwächter, J., Eckart, A., et al. 2013, A\&A, 558, A34

Bruzual, G., \& Charlot, S. 1993, ApJ, 405, 538

Bruzual, G., \& Charlot, S. 2003, MNRAS, 344, 1000

Buta, R., van Driel, W., Braine, J., et al. 1995, ApJ, 450, 593

Capetti, A., de Ruiter, H. R., Fanti, R., et al. 2000, A\&A, 362, 871

Cappellari, M., McDermid, R. M., Alatalo, K., et al. 2012, Natur, 484, 485

Cenarro, A. J., Gorgas, J., Vazdekis, A., Cardiel, N., \& Peletier, R. F. 2003, MNRAS, 339, L12

Cesetti, M., Ivanov, V. D., Morelli, L., et al. 2009, A\&A, 497, 41

Cid Fernandes, R., González Delgado, R. M., Schmitt, H., et al. 2004, ApJ, 605,105

Cid Fernandes, R., González Delgado, R. M., Storchi-Bergmann, T., Martins, L. P., \& Schmitt, H. 2005, MNRAS, 356, 270

Cid Fernandes, R., Mateus, A., Sodré, L., Stasińska, G., \& Gomes, J. M. 2005, MNRAS, 358, 363

Cisternas, M., Gadotti, D. A., Knapen, J. H., et al. 2013, ApJ, 776, 50

Claussen, M. J., Diamond, P. J., Braatz, J. A., Wilson, A. S., \& Henkel, C. 1998, ApJL, 500, L129

Colbert, E. J. M., \& Mushotzky, R. F. 1999, ApJ, 519, 89

Combes, F., Baker, A. J., Schinnerer, E., et al. 2009, A\&A, 503, 73

Combes, F., Braine, J., Casoli, F., Gerin, M., \& van Driel, W. 1992, A\&A, 259, L65

Combes, F., García-Burillo, S., Boone, F., et al. 2004, A\&A, 414, 857

Condon, J. J., Frayer, D. T., \& Broderick, J. J. 1991, AJ, 101, 362

Conroy, C., \& van Dokkum, P. 2012, ApJ, 747, 69

Cotton, D. V., Bailey, J., \& Kedziora-Chudczer, L. 2014, MNRAS, 439, 387

Cotton, W. D., Feretti, L., Giovannini, G., Lara, L., \& Venturi, T. 1999, ApJ, 519, 108 
Crocker, A. F., Bureau, M., Young, L. M., \& Combes, F. 2008, MNRAS, 386, 1811

Dale, D. A., Smith, J. D. T., Armus, L., et al. 2006, ApJ, 646, 161

Davies, R. I., Müller Sánchez, F., Genzel, R., et al. 2007, ApJ, 671, 1388

Davies, R. I., Sternberg, A., Lehnert, M. D., \& Tacconi-Garman, L. E. 2005, ApJ, 633, 105

Davies, R. I., Tacconi, L. J., \& Genzel, R. 2004, ApJ, 602, 148

Davis, G. R., Naylor, D. A., Griffin, M. J., Clark, T. A., \& Holland, W. S. 1997, Icar, 130, 387

de Lorenzo-Cáceres, A., Falcón-Barroso, J., \& Vazdekis, A. 2013, MNRAS, 431, 2397

de Vaucouleurs, G. 1975, Nearby Groups of Galaxies (Chicage, IL: Univ. Chicago Press

Devereux, N., Ford, H., \& Jacoby, G. 1997, ApJL, 481, L71

Doi, A., Kohno, K., Nakanishi, K., et al. 2013, ApJ, 765, 63

Donato, D., Sambruna, R. M., \& Gliozzi, M. 2004, ApJ, 617, 915

Dopita, M. A., \& Sutherland, R. S. 1996, ApJS, 102, 161

Doyon, R., Joseph, R. D., \& Wright, G. S. 1989, in ESA Special Publication 290, Infrared Spectroscopy in Astronomy, ed. E. Böhm-Vitense, 477

Dudik, R. P., Satyapal, S., Gliozzi, M., \& Sambruna, R. M. 2005, ApJ, 620, 113

Dudik, R. P., Satyapal, S., \& Marcu, D. 2009, ApJ, 691, 1501

Elias, J. H., Joyce, R. R., Liang, M., et al. 2006, SPIE, 62694C

Elitzur, M., \& Shlosman, I. 2006, ApJL, 648, L101

Eracleous, M., Hwang, J. A., \& Flohic, H. M. L. G. 2010, ApJ, 711, 796

Eracleous, M., Hwang, J. A., \& Flohic, H. M. L. G. 2010, ApJS, 187, 135

Eracleous, M., Shields, J. C., Chartas, G., \& Moran, E. C. 2002, ApJ, 565,108

Erwin, P. 2004, A\&A, 415, 941

Esin, A. A., McClintock, J. E., \& Narayan, R. 1997, ApJ, 489, 865

Falcke, H., Nagar, N. M., Wilson, A. S., \& Ulvestad, J. S. 2000, ApJ, 542, 197

Falcón-Barroso, J., Bacon, R., Bureau, M., et al. 2006, MNRAS, 369, 529

Ferland, G. J., \& Netzer, H. 1983, ApJ, 264, 105

Ferruit, P., Wilson, A. S., \& Mulchaey, J. 2000, ApJS, 128, 139

Filho, M. E., Barthel, P. D., \& Ho, L. C. 2002, A\&A, 385, 425

Filho, M. E., Barthel, P. D., \& Ho, L. C. 2006, A\&A, 451, 71

Filippenko, A. V., \& Sargent, W. L. W. 1989, ApJL, 342, L11

Filippenko, A. V., \& Sargent, W. L. W. 1992, AJ, 103, 28

Flohic, H. M. L. G., Eracleous, M., Chartas, G., Shields, J. C., \& Moran, E. C. 2006, ApJ, 647, 140

Forbes, D. A., \& Ward, M. J. 1993, ApJ, 416, 150

Gabel, J. R., \& Bruhweiler, F. C. 2002, AJ, 124, 737

García-Burillo, S., Fernández-García, S., Combes, F., et al. 2009, A\&A, 496, 85

Geballe, T. R., Mason, R. E., Rodríguez-Ardila, A., \& Axon, D. J. 2009, ApJ, 701, 1710

Genzel, R., Weitzel, L., \& Tacconi-Garman, L. E. 1995, ApJ, 444, 129

Giovannini, G., Cotton, W. D., Feretti, L., Lara, L., \& Venturi, T. 2001, ApJ, 552,508

Glass, I. S. 1999, in Handbook of Infrared Astronomy, ed. R. Ellis et al. (New York: Cambridge Univ. Press), 27

Goldader, J. D., Joseph, R. D., Doyon, R., \& Sanders, D. B. 1997a, ApJS, 108,449

Goldader, J. D., Joseph, R. D., Doyon, R., \& Sanders, D. B. 1997b, ApJ, 474, 104

González Delgado, R. M., Cid Fernandes, R., Pérez, E., et al. 2004, ApJ, 605,127

González Delgado, R. M., Leitherer, C., \& Heckman, T. M. 1999, ApJS, 125,489

Gonzalez Delgado, R. M., \& Perez, E. 1996, MNRAS, 281, 1105

González Delgado, R. M., Pérez, E., \& Cid Fernandes, R. 2014, A\&A, 562, A47

González Delgado, R. M., Pérez, E., Cid Fernandes, R., \& Schmitt, H. 2008, AJ, 135, 747

Gonzalez-Martin, O., Diaz-Gonzalez, D., Acosta-Pulido, J. A., et al. 2014, A\&A, 567, A92

González-Martín, O., Masegosa, J., Márquez, I., \& Guainazzi, M. 2009, ApJ, 704,1570

González-Martín, O., Masegosa, J., Márquez, I., Guainazzi, M., \& Jiménez-Bailón, E. 2009, A\&A, 506, 1107

González-Martín, O., Masegosa, J., Márquez, I., Guerrero, M. A., \& Dultzin-Hacyan, D. 2006, A\&A, 460, 45

Goodrich, R. W., Veilleux, S., \& Hill, G. J. 1994, ApJ, 422, 521

Goulding, A. D., \& Alexander, D. M. 2009, MNRAS, 398, 1165

Greenhill, L. J., Jiang, D. R., Moran, J. M., et al. 1995, ApJ, 440, 619

Gullikson, K., Dodson-Robinson, S., \& Kraus, A. 2014, AJ, 148, 53
Gutiérrez, C. M., López-Corredoira, M., Prada, F., \& Eliche, M. C. 2002, ApJ, 579,592

Habing, H. J., \& Olofsson, H. (ed.) 2003, Asymptotic Giant Branch Stars (Berlin: Springer), 15

Hada, K., Doi, A., Nagai, H., et al. 2013, ApJ, 779, 6

Hagiwara, Y. 2007, AJ, 133, 1176

Hagiwara, Y., Henkel, C., Menten, K. M., \& Nakai, N. 2001, ApJL, 560, L37

Halderson, E. L., Moran, E. C., Filippenko, A. V., \& Ho, L. C. 2001, AJ, 122,637

Hawarden, T. G., Israel, F. P., Geballe, T. R., \& Wade, R. 1995, MNRAS, 276, 1197

Hawarden, T. G., Ryder, S. D., Massey, R. J., Wright, G. S., \& Takamiya, M. 1999, Ap\&SS, 269, 501

Heckman, T. M. 1980, A\&A, 87, 152

Helou, G., Salpeter, E. E., \& Terzian, Y. 1982, AJ, 87, 1443

Henkel, C., Guesten, R., Downes, D., Thum, C., Wilson, T. L., \& Biermann, P. 1984, A\&A, 141, L1

Hernández-García, L., González-Martín, O., Márquez, I., \& Masegosa, J. 2013, A\&A, 556, A47

Hicks, E. K. S., Davies, R. I., Maciejewski, W., et al. 2013, ApJ, 768, 107

Higdon, J. L., Buta, R. J., \& Purcell, G. B. 1998, AJ, 115, 80

Ho, L. C. 2008, ARA\&A, 46, 475

Ho, L. C. 2009, ApJ, 699, 626

Ho, L. C., Feigelson, E. D., Townsley, L. K., et al. 2001, ApJL, 549, L51

Ho, L. C., Filippenko, A. V., \& Sargent, W. L. W. 1995, ApJS, 98, 477

Ho, L. C., Filippenko, A. V., \& Sargent, W. L. W. 1996, ApJ, 462, 183

Ho, L. C., Filippenko, A. V., \& Sargent, W. L. W. 1997, ApJS, 112, 315

Ho, L. C., Filippenko, A. V., \& Sargent, W. L. W. 2003, ApJ, 583, 159

Ho, L. C., Filippenko, A. V., Sargent, W. L. W., \& Peng, C. Y. 1997, ApJS, 112,391

Ho, L. C., Greene, J. E., Filippenko, A. V., \& Sargent, W. L. W. 2009, ApJS, 183,1

Ho, L. C., Rudnick, G., Rix, H.-W., et al. 2000, ApJ, 541, 120

Ho, L. C., \& Ulvestad, J. S. 2001, ApJS, 133, 77

Ho, L. C., van Dyk, S. D., Pooley, G. G., Sramek, R. A., \& Weiler, K. W 1999, AJ, 118, 843

Ho, P. T. P., Martin, R. N., Henkel, C., \& Turner, J. L. 1987, ApJ, 320, 663 Houghton, R. C. W., Magorrian, J., Sarzi, M., et al. 2006, MNRAS, 367, 2

Hudson, M. J., Lucey, J. R., Smith, R. J., Schlegel, D. J., \& Davies, R. L. 2001, MNRAS, 327, 265

Hummel, E., van der Hulst, J. M., \& Dickey, J. M. 1984, A\&A, 134, 207

Humphreys, E. M. L., Reid, M. J., Moran, J. M., Greenhill, L. J., \& Argon, A. L. 2013, ApJ, 775, 13

Imanishi, M., \& Alonso-Herrero, A. 2004, ApJ, 614, 122

Imanishi, M., \& Wada, K. 2004, ApJ, 617, 214

Irwin, J. A., Seaquist, E. R., Taylor, A. R., \& Duric, N. 1987, ApJL, 313, L91

Israel, F. P., van der Werf, P. P., Hawarden, T. G., \& Aspin, C. 1998, A\&A, 336,433

Ivanov, V. D., Rieke, G. H., Groppi, C. E., et al. 2000, ApJ, 545, 190

Ivanov, V. D., Rieke, M. J., Engelbracht, C. W., et al. 2004, ApJS, 151, 387

Iwasawa, K., Fabian, A. C., Almaini, O., et al. 2000, MNRAS, 318, 879

Kang, W.-R., Woo, J.-H., Schulze, A., et al. 2013, ApJ, 767, 26

Kannappan, S. J., \& Gawiser, E. 2007, ApJL, 657, L5

Kawara, K., Gregory, B., \& Nishida, M. 1987, ApJL, 321, L35

Keel, W. C. 1996, PASP, 108, 917

Keel, W. C., \& Hummel, E. 1988, A\&A, 194, 90

Knop, R. A., Armus, L., Matthews, K., Murphy, T. W., \& Soifer, B. T. 2001 , AJ, 122, 764

Komossa, S., Böhringer, H., \& Huchra, J. P. 1999, A\&A, 349, 88

Kong, X., Zhou, X., Chen, J., et al. 2000, AJ, 119, 2745

Kormendy, J., \& Ho, L. C. 2013, ARA\&A, 51, 511

Kotilainen, J. K., Hyvönen, T., Reunanen, J., \& Ivanov, V. D. 2012, MNRAS, 425, 1057

Kraemer, S. B., Ho, L. C., Crenshaw, D. M., Shields, J. C., \& Filippenko, A. V. 1999, ApJ, 520, 564

Krause, M., Fendt, C., \& Neininger, N. 2007, A\&A, 467, 1037

Kriek, M., Labbé, I., Conroy, C., et al. 2010, ApJL, 722, L64

Krips, M., Eckart, A., Krichbaum, T. P., et al. 2007, A\&A, 464, 553

Krips, M., Eckart, A., Neri, R., et al. 2005, A\&A, 442, 479

Kuntschner, H., Emsellem, E., Bacon, R., et al. 2010, MNRAS, 408, 97

Kuo, C. Y., Braatz, J. A., Condon, J. J., et al. 2011, ApJ, 727, 20

La Barbera, F., Ferreras, I., Vazdekis, A., et al. 2013, MNRAS, 433, 3017

Laine, S., Appleton, P. N., Gottesman, S. T., Ashby, M. L. N., \& Garland, C. A. 2010, AJ, 140, 753

Laine, S., van der Marel, R. P., Lauer, T. R., et al. 2003, AJ, 125, 478

Lamers, H. J. G. L. M., Panagia, N., Scuderi, S., et al. 2002, ApJ, 566, 818 
Lançon, A., \& Mouhcine, M. 2002, A\&A, 393, 167

Landt, H., Bentz, M. C., Ward, M. J., et al. 2008, ApJS, 174, 282

Landt, H., Elvis, M., Ward, M. J., et al. 2011, MNRAS, 414, 218

Landt, H., Ward, M. J., Peterson, B. M., et al. 2013, MNRAS, 432, 113

Larkin, J. E., Armus, L., Knop, R. A., Soifer, B. T., \& Matthews, K. 1998 ApJS, 114, 59

Lauer, T. R. 1988, ApJ, 325, 49

Laurikainen, E., Salo, H., \& Buta, R. 2005, MNRAS, 362, 1319

Lira, P., Lawrence, A., O'Brien, P., et al. 1999, MNRAS, 305, 109

Liuzzo, E., Giovannini, G., Giroletti, M., \& Taylor, G. B. 2010, A\&A, 516, A1 Lord, S. D. 1992, NASA Technical Memor.103957

Loubser, S. I., Sánchez-Blázquez, P., Sansom, A. E., \& Soechting, I. K. 2009, MNRAS, 398, 133

Lyubenova, M., Kuntschner, H., Rejkuba, M., et al. 2012, A\&A, 543, A75

Macchetto, F., Pastoriza, M., Caon, N., et al. 1996, A\&AS, 120, 463

Macri, L. M., Stanek, K. Z., Bersier, D., Greenhill, L. J., \& Reid, M. J. 2006, ApJ, 652, 1133

Mager, V. A., Madore, B. F., \& Freedman, W. L. 2008, ApJ, 689, 721

Malkan, M. A., Gorjian, V., \& Tam, R. 1998, ApJS, 117, 25

Mannucci, F., Basile, F., Poggianti, B. M., et al. 2001, MNRAS, 326, 745

Maoz, D., Koratkar, A., Shields, J. C., et al. 1998, AJ, 116, 55

Maoz, D., Nagar, N. M., Falcke, H., \& Wilson, A. S. 2005, ApJ, 625, 699

Maraston, C. 2005, MNRAS, 362, 799

Maraston, C., Daddi, E., Renzini, A., et al. 2006, ApJ, 652, 85

Marconi, A., van der Werf, P. P., Moorwood, A. F. M., \& Oliva, E. 1996, A\&A, 315, 335

Marigo, P., Girardi, L., Bressan, A., et al. 2008, A\&A, 482, 883

Márquez, I., Masegosa, J., Durret, F., et al. 2003, A\&A, 409, 459

Martel, A. R., Ford, H. C., Bradley, L. D., et al. 2004, AJ, 128, 2758

Martini, P., Regan, M. W., Mulchaey, J. S., \& Pogge, R. W. 2003, ApJS, 146,353

Martins, L. P., Rodríguez-Ardila, A., de Souza, R., \& Gruenwald, R. 2010, MNRAS, 406, 2168

Martins, L. P., Rodríguez-Ardila, A., Diniz, S., Gruenwald, R., \& de Souza, R. 2013, MNRAS, 431, 1823

Martins, L. P., Rodríguez-Ardila, A., Diniz, S., Riffel, R., \& de Souza, R. 2013, MNRAS, 435, 2861

Mason, R. E., Lopez-Rodriguez, E., Packham, C., et al. 2012, AJ, 144, 11

Mason, R. E., Ramos Almeida, C., Levenson, N. A., Nemmen, R., \& Alonso-Herrero, A. 2013, ApJ, 777, 164

Matsuoka, Y., Yuan, F.-T., Takeuchi, Y., \& Yanagisawa, K. 2012, PASJ, 64, 44

Matt, G., Bianchi, S., Guainazzi, M., Barcons, X., \& Panessa, F. 2012, A\&A, 540, A111

Mazzalay, X., Maciejewski, W., Erwin, P., et al. 2014, MNRAS, 438, 2036

Mazzalay, X., Saglia, R. P., Erwin, P., et al. 2013, MNRAS, 428, 2389

McLeod, K. K., Rieke, G. H., Rieke, M. J., \& Kelly, D. M. 1993, ApJ, 412, 111

Melbourne, J., Williams, B. F., Dalcanton, J. J., et al. 2012, ApJ, 748, 47

Mezcua, M., \& Prieto, M. A. 2014, ApJ, 787, 62

Miner, J., Rose, J. A., \& Cecil, G. 2011, ApJL, 727, L15

Minezaki, T., Yoshii, Y., Kobayashi, Y., et al. 2006, ApJL, 643, L5

Miyoshi, M., Moran, J., Herrnstein, J., et al. 1995, Natur, 373, 127

Mobasher, B., \& James, P. A. 1996, MNRAS, 280, 895

Monaco, L., Saviane, I., Perina, S., et al. 2009, A\&A, 502, L9

Moorwood, A. F. M., \& Oliva, E. 1988, A\&A, 203, 278

Mor, R., \& Netzer, H. 2012, MNRAS, 420, 526

Moran, E. C., Eracleous, M., Leighly, K. M., et al. 2005, AJ, 129, 2108

Moran, E. C., Filippenko, A. V., Ho, L. C., et al. 1999, PASP, 111, 801

Mould, J., Reynolds, T., Readhead, T., et al. 2012, ApJS, 203, 14

Mouri, H. 1994, ApJ, 427, 777

Mouri, H., Nishida, M., Taniguchi, Y., \& Kawara, K. 1990, ApJ, 360, 55

Nagar, N. M., Falcke, H., \& Wilson, A. S. 2005, A\&A, 435, 521

Nagar, N. M., Falcke, H., Wilson, A. S., \& Ho, L. C. 2000, ApJ, 542, 186

Nagar, N. M., Falcke, H., Wilson, A. S., \& Ulvestad, J. S. 2002, A\&A, 392, 53

Narayan, R., \& McClintock, J. E. 2008, NewAR, 51, 733

Naylor, D. A., Chapman, I. M., \& Gom, B. G. 2002, SPIE, 4815, 36

Nelson, C. H., Green, R. F., Bower, G., Gebhardt, K., \& Weistrop, D. 2004, ApJ, 615, 652

Noordermeer, E., van der Hulst, J. M., Sancisi, R., Swaters, R. A., \& van Albada, T. S. 2005, A\&A, 442, 137

Nyland, K., Marvil, J., Wrobel, J. M., Young, L. M., \& Zauderer, B. A. 2012, ApJ, 753, 103

Ohyama, Y., \& Taniguchi, Y. 1996, in ASP Conf. Ser. 103, The Physics of Liners in View of Recent Observations, ed. M. Eracleous et al. (San Francisco, CA: ASP), 205
Oi, N., Imanishi, M., \& Imase, K. 2010, PASJ, 62, 1509

Oliva, E., Salvati, M., Moorwood, A. F. M., \& Marconi, A. 1994, A\&A, 288,457

Origlia, L., Moorwood, A. F. M., \& Oliva, E. 1993, A\&A, 280, 536

Osterbrock, D. E., \& Martel, A. 1993, ApJ, 414, 552

Palacios, J., Garcia-Vargas, M. L., Diaz, A., Terlevich, R., \& Terlevich, E. 1997, A\&A, 323, 749

Panessa, F., Bassani, L., Cappi, M., et al. 2006, A\&A, 455, 173

Panessa, F., de Rosa, A., Bassani, L., et al. 2011, MNRAS, 417, 2426

Paturel, G., Petit, C., Prugniel, P., et al. 2003, A\&A, 412, 45

Pecaut, M. J., \& Mamajek, E. E. 2013, ApJS, 208, 9

Peletier, R. F., Falcón-Barroso, J., Bacon, R., et al. 2007, MNRAS, 379, 445

Pereira-Santaella, M., Alonso-Herrero, A., Santos-Lleo, M., et al. 2011, A\&A, 535, A93

Pérez, I., \& Sánchez-Blázquez, P. 2011, A\&A, 529, A64

Peterson, B. M., Bentz, M. C., Desroches, L.-B., et al. 2005, ApJ, 632, 799

Phillips, M. M., \& Malin, D. F. 1982, MNRAS, 199, 905

Pogge, R. W. 1988, ApJ, 332, 702

Prieto, M. A., \& Viegas, S. M. 2000, ApJ, 532, 238

Proctor, R. N., \& Sansom, A. E. 2002, MNRAS, 333, 517

Proctor, R. N., Sansom, A. E., \& Reid, I. N. 2000, MNRAS, 311, 37

Quataert, E., di Matteo, T., Narayan, R., \& Ho, L. C. 1999, ApJL, 525, L89

Radford, S. J. E. 2011, RMxAA, 41, 87

Raimann, D., Storchi-Bergmann, T., Bica, E., \& Alloin, D. 2001, MNRAS, 324, 1087

Ramos Almeida, C., Levenson, N. A., Rodríguez Espinosa, J. M., et al. 2009, ApJ, 702, 1127

Ramos Almeida, C., Pérez García, A. M., \& Acosta-Pulido, J. A. 2009, ApJ, 694, 1379

Rayner, J. T., Cushing, M. C., \& Vacca, W. D. 2009, ApJS, 185, 289

Reunanen, J., Kotilainen, J. K., \& Prieto, M. A. 2002, MNRAS, 331, 154

Rhee, J. H., \& Larkin, J. E. 2005, ApJ, 620, 151

Rickes, M. G., Pastoriza, M. G., \& Bonatto, C. 2009, A\&A, 505, 73

Rieke, G. H., Lebofsky, M. J., Thompson, R. I., Low, F. J., \& Tokunaga, A. T. 1980, ApJ, 238, 24

Riffel, R., Bonatto, C., Cid Fernandes, R., Pastoriza, M. G., \& Balbinot, E. 2011, MNRAS, 411, 1897

Riffel, R., Pastoriza, M. G., Rodríguez-Ardila, A., \& Bonatto, C. 2009, MNRAS, 400, 273

Riffel, R., Pastoriza, M. G., Rodríguez-Ardila, A., \& Maraston, C. 2007, ApJL, 659, L103

Riffel, R., Rodríguez-Ardila, A., Aleman, I., et al. 2013, MNRAS, 430, 2002

Riffel, R., Rodríguez-Ardila, A., \& Pastoriza, M. G. 2006, A\&A, 457, 61

Riffel, R., Ruschel-Dutra, D., Pastoriza, M. G., et al. 2011, MNRAS, 410, 2714

Riffel, R. A., Ho, L. C., Mason, R., et al. 2015, MNRAS, 446, 2823

Riffel, R. A., Storchi-Bergmann, T., \& Nagar, N. M. 2010, MNRAS, 404, 166

Riffel, R. A., Storchi-Bergmann, T., Riffel, R., et al. 2013, MNRAS, 429, 2587

Roche, P. F., Aitken, D. K., Smith, C. H., \& Ward, M. J. 1991, MNRAS, 248, 606

Rodríguez-Ardila, A., Binette, L., Pastoriza, M. G., \& Donzelli, C. J. 2000, ApJ, 538, 581

Rodríguez-Ardila, A., \& Mazzalay, X. 2006, MNRAS, 367, L57

Rodríguez-Ardila, A., Pastoriza, M. G., Viegas, S., Sigut, T. A. A., \& Pradhan, A. K. 2004, A\&A, 425, 457

Rodríguez-Ardila, A., Prieto, M. A., Portilla, J. G., \& Tejeiro, J. M. 2011, ApJ, 743,100

Rodríguez-Ardila, A., Prieto, M. A., Viegas, S., \& Gruenwald, R. 2006, ApJ, 653, 1098

Rodríguez-Ardila, A., Riffel, R., \& Pastoriza, M. G. 2005, MNRAS, 364, 1041

Rodríguez-Ardila, A., Viegas, S. M., Pastoriza, M. G., \& Prato, L. 2002, ApJ, 565,140

Sánchez-Blázquez, P., Gorgas, J., Cardiel, N., \& González, J. J. 2006, A\&A, 457,809

Sandage, A., \& Bedke, J. 1994, in The Carnegie Atlas of Galaxies, Vol. I, II, (Washington, DC: Carnegie Institution of Washington Publ.)

Sanders, D. B., Mazzarella, J. M., Kim, D.-C., Surace, J. A., \& Soifer, B. T. 2003, AJ, 126, 1607

Sanmartim, D., Storchi-Bergmann, T., \& Brotherton, M. S. 2014, MNRAS, 443,584

Sarzi, M., Allard, E. L., Knapen, J. H., \& Mazzuca, L. M. 2007, MNRAS, 380, 949

Sarzi, M., Rix, H.-W., Shields, J. C., et al. 2005, ApJ, 628, 169

Sarzi, M., Shields, J. C., Schawinski, K., et al. 2010, MNRAS, 402, 2187

Satyapal, S., Dudik, R. P., O’Halloran, B., \& Gliozzi, M. 2005, ApJ, 633, 86 Schiavon, R. P. 2007, ApJS, 171, 146

Seifahrt, A., Käufl, H. U., Zängl, G., et al. 2010, A\&A, 524, A11 
Serra, P., Trager, S. C., Oosterloo, T. A., \& Morganti, R. 2008, A\&A, 483, 57

Seth, A. C., Cappellari, M., Neumayer, N., et al. 2010, ApJ, 714, 713

Shields, J. C., Rix, H.-W., McIntosh, D. H., et al. 2000, ApJL, 534, L27

Sil'chenko, O. K., \& Afanasiev, V. L. 2006, AstL, 32, 534

Simpson, C., Forbes, D. A., Baker, A. C., \& Ward, M. J. 1996, MNRAS, 283, 777

Singh, R., van de Ven, G., Jahnke, K., et al. 2013, A\&A, 558, A43

Skelton, J. E., Lawrence, A., Pappa, A., Lira, P., \& Almaini, O. 2005, MNRAS, 358, 781

Skrutskie, M. F., Cutri, R. M., Stiening, R., et al. 2006, AJ, 131, 1163

Smith, B. J., Giroux, M. L., Struck, C., \& Hancock, M. 2010, AJ, 139, 1212

Smith, J. D. T., Dale, D. A., Armus, L., et al. 2004, ApJS, 154, 199

Smith, R. J., Lucey, J. R., \& Carter, D. 2012, MNRAS, 426, 2994

Sosa-Brito, R. M., Tacconi-Garman, L. E., Lehnert, M. D., \& Gallimore, J. F. 2001, ApJS, 136, 61

Sparke, L. S., van Moorsel, G., Erwin, P., \& Wehner, E. M. H. 2008, AJ, 135,99

Sparke, L. S., van Moorsel, G., Schwarz, U. J., \& Vogelaar, M. 2009, AJ, 137, 3976

Spiniello, C., Trager, S., Koopmans, L. V. E., \& Conroy, C. 2014, MNRAS, 438, 1483

Stasińska, G., Vale Asari, N., Cid Fernandes, R., et al. 2008, MNRAS, 391, 129

Storchi-Bergmann, T., Fernandes, R. C., \& Schmitt, H. R. 1998, ApJ, 501, 94

Struve, C., Oosterloo, T., Sancisi, R., Morganti, R., \& Emonts, B. H. C. 2010, A\&A, 523, A75

Taam, R. E., Liu, B. F., Yuan, W., \& Qiao, E. 2012, ApJ, 759, 65

Taniguchi, Y., Ohyama, Y., Yamada, T., Mouri, H., \& Yoshida, M. 1996, ApJ, 467,215

Telesco, C. M., Gatley, I., \& Stewart, J. M. 1982, ApJL, 263, L13

Terashima, Y., Iyomoto, N., Ho, L. C., \& Ptak, A. F. 2002, ApJS, 139, 1

Terashima, Y., \& Wilson, A. S. 2003, ApJ, 583, 145

Terlevich, A. I., \& Forbes, D. A. 2002, MNRAS, 330, 547

Terlevich, R., \& Melnick, J. 1985, MNRAS, 213, 841

Thomas-Osip, J., McWilliam, A., Phillips, M. M., et al. 2007, PASP, 119, 697

Thompson, R. I. 1996, ApJL, 459, L61
Tran, H. D., Lyke, J. E., \& Mader, J. A. 2011, ApJL, 726, L21

Tully, R. B., Shaya, E. J., \& Pierce, M. J. 1996, yCat, 208, 479

Turnbull, A. J., Bridges, T. J., \& Carter, D. 1999, MNRAS, 307, 967

Valluri, M., Ferrarese, L., Merritt, D., \& Joseph, C. L. 2005, ApJ, 628, 137

van den Bergh, S. 1976, ApJ, 206, 883

van der Laan, T. P. R., Schinnerer, E., Böker, T., \& Armus, L. 2013, A\&A, 560, A99

van Dokkum, P. G., \& Conroy, C. 2010, Natur, 468, 940

Vanzi, L., \& Rieke, G. H. 1997, ApJ, 479, 694

Veilleux, S., Goodrich, R. W., \& Hill, G. J. 1997, ApJ, 477, 631

Venturi, T., Giovannini, G., Feretti, L., Comoretto, G., \& Wehrle, A. E. 1993, ApJ, 408, 81

Vermeulen, R. C., Ros, E., Kellermann, K. I., et al. 2003, A\&A, 401, 113

Véron-Cetty, M.-P., \& Véron, P. 2006, A\&A, 455, 773

Vollmer, B., Balkowski, C., Cayatte, V., van Driel, W., \& Huchtmeier, W. 2004, A\&A, 419, 35

Vollmer, B., Beckert, T., \& Davies, R. I. 2008, A\&A, 491, 441

Vollmer, B., Cayatte, V., Boselli, A., Balkowski, C., \& Duschl, W. J. 1999, A\&A, 349, 411

Walker, C. E., Lebofsky, M. J., \& Rieke, G. H. 1988, ApJ, 325, 687

Walsh, J. L., van den Bosch, R. C. E., Barth, A. J., \& Sarzi, M. 2012, ApJ, 753, 79

Watson, W. D., \& Wallin, B. K. 1994, ApJL, 432, L35

Whitmore, B. C., Lucas, R. A., McElroy, D. B., et al. 1990, AJ, 100, 1489

Wiklind, T., Combes, F., \& Henkel, C. 1995, A\&A, 297, 643

Wilson, A. S., Braatz, J. A., \& Henkel, C. 1995, ApJL, 455, L127

Wrobel, J. M., \& Ho, L. C. 2006, ApJL, 646, L95

Younes, G., Porquet, D., Sabra, B., Reeves, J. N., \& Grosso, N. 2012, A\&A, 539, A104

Yu, Z., Yuan, F., \& Ho, L. C. 2011, ApJ, 726, 87

Yun, M. S., Ho, P. T. P., \& Lo, K. Y. 1994, Natur, 372, 530

Zhang, Y., Gu, Q., \& Ho, L. C. 2008, A\&A, 487, 177

Zibetti, S., Gallazzi, A., Charlot, S., Pierini, D., \& Pasquali, A. 2013, MNRAS, 428, 1479

Zwicky, F., et al. 1996, yCat, 7190, 0 